Validation of the International Reading Speed Texts in a Canadian Sample

Elliott Morrice

A Thesis

In

The Department

Of

Psychology

Presented in Partial Fulfillment of the Requirements

For the Degree of Master of Arts (Psychology) at

Concordia University

Montreal, Quebec, Canada

April 2017

(C) Elliott Morrice, 2017 


\section{CONCORDIA UNIVERSITY \\ School of Graduate Studies}

This is to certify that the thesis prepared

By: $\quad$ Elliott Morrice

Entitled: $\quad$ Validation of the International Reading Speed Texts i

and submitted in partial fulfillment of the requirements for the degree of

\section{A Psychology (Research and Clinical Training Option}

complies with the regulations of the University and meets the accepted standards with respect to originality and quality.

Signed by the final examining committee:

\begin{tabular}{ll} 
Dr. Andrew Chapman & Chair \\
Dr. Natalie Philips & Examiner \\
\hline Dr. Walter Wittich & Examiner \\
\hline Dr. Aaron Johnson & Supervisor
\end{tabular}

Approved by

Chair of Department or Graduate Program Director

Dean of Faculty

Date 


\begin{abstract}
Validation of the International Reading Speed Texts

in a Canadian Sample
\end{abstract}

\title{
Elliott Morrice
}

The English language IReST is a measure of continuous reading, developed and normalized in the United Kingdom (UK) that is used to assess reading speed in normally sighted and visually impaired individuals. However, the IReST is used in rehabilitations settings across North America (NA) to assess reading speed despite not having been validated in an Englishspeaking NA sample. In addition to not having been validated in NA, the IReST has also not been validated in a sample of individuals with visual impairments; therefore the purpose of this thesis was to validate the English Language IReST in a NA sample, as well as examine the impact of a simulated visual impairment on reading speed on the IReST. As a high proportion of NA is bilingual, this thesis also examined the impact of language background and fluency on the IReST. Finally, as low vision rehabilitation specialists may not have received training on the administration of the IReST, there is the potential for administration errors; thus this thesis also examined the impact of improper administration on reading speeds on the IReST. The results of this the thesis found that: (1) the reading speeds of English-speaking NAs fell outside of the normative values of the UK English IReST; (2) simulated visual impairments significantly reduce reading speeds on the IReST; (3) assessing individuals in their non-dominant, yet fluent language, does not significantly impact reading speeds on the IReST; and (4) improper administration of the IReST significantly reduces measured reading speed on the IReST. 


\section{Acknowledgements}

I would like to take this opportunity to thank my supervisor, Dr. Aaron Johnson, for his guidance and support over the past four years; for allowing me become a member of his lab, providing me with research opportunities, and by placing his faith in me by accepting me into the clinical program. I would also like to thank my co-supervisor, Dr. Walter Wittich, for assistance, encouragement, and for teaching me to "Grab the bull by the balls," and follow my passion. This work would not have been possible without you.

I would also like to take this opportunity to thank the members of the Concordia Vision Labs; in particular I would like to thank Mr. Julian Hughes, a dedicated undergraduate thesis student and research assistant whose support has been invaluable. Finally, I would like my friends and family who have supported me along this journey, and, most importantly my soon-tobe husband Jacob Le Gallais, who has been by my side throughout this process. Thank you. 
Table of Contents

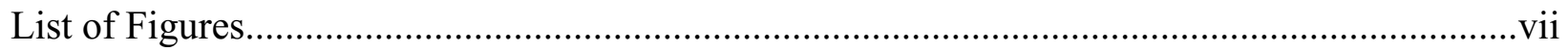

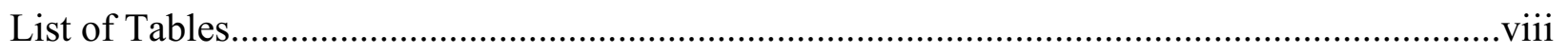

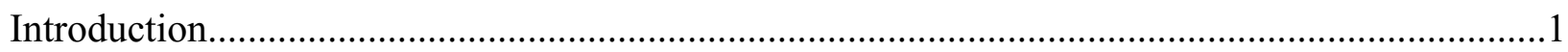

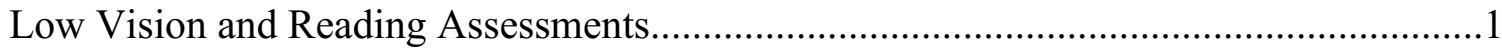

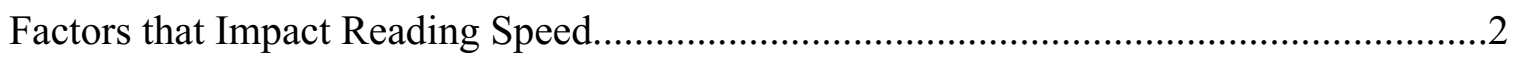

Objective Versus Subjective Measures of Reading Speed...........................................4

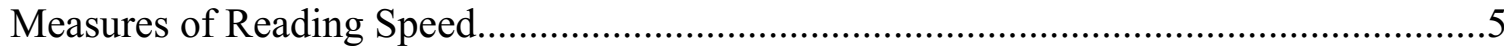

The International Reading Speed Texts (IReST) ....................................................6

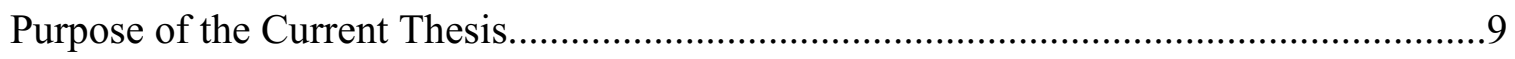

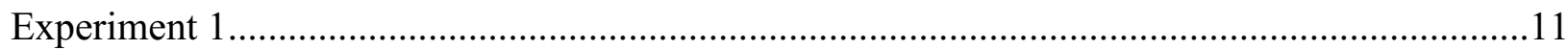

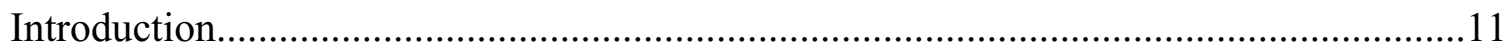

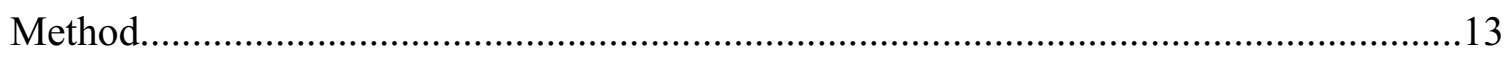

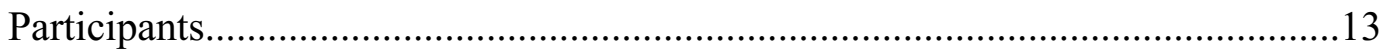

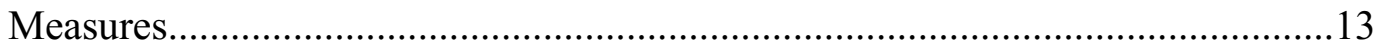

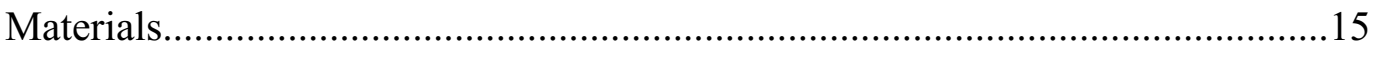

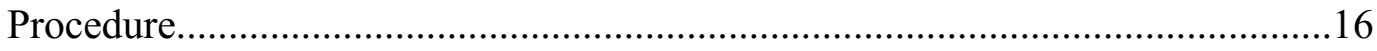

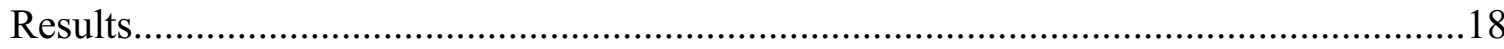

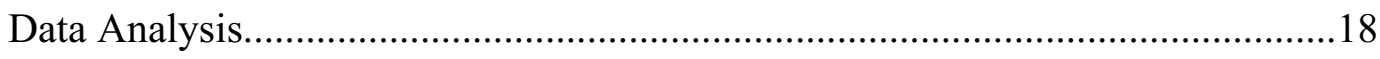

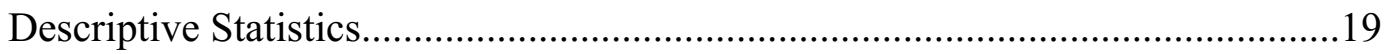

Visual Acuity and Low Vision Simulator Goggles..........................................21

NA Sample Compared to Normative IReST Data...........................................21

Normal Vision Condition VS. Impaired Condition...........................................24

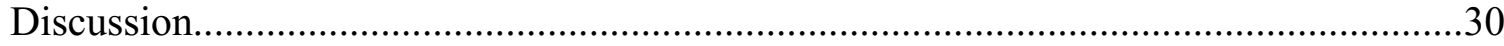

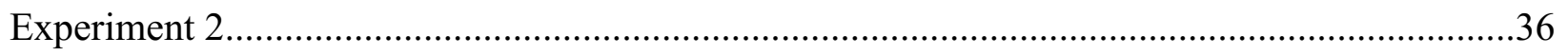

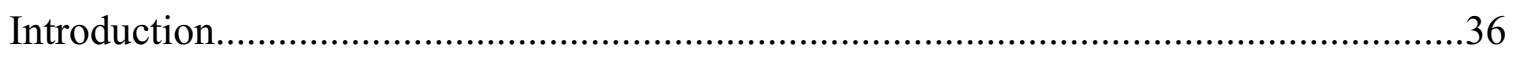

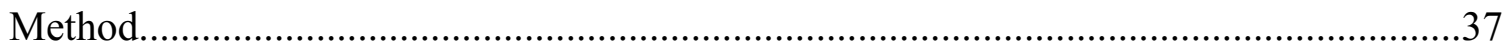

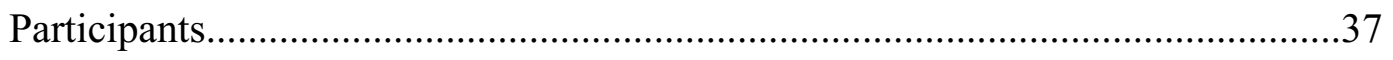

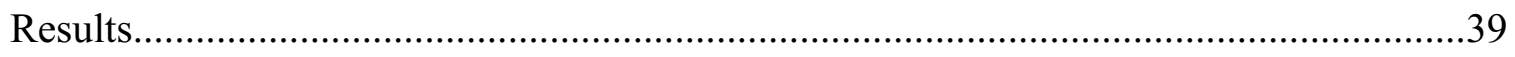

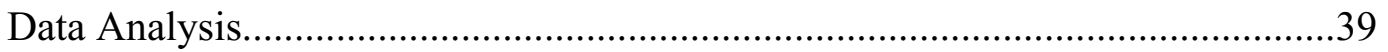

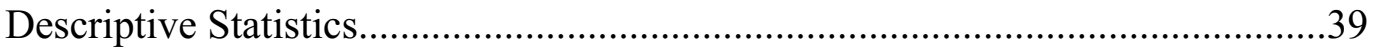


Visual Acuity and Low Vision Simulator Goggles................................................42

Multilingual Sample Compared to the Normative NA IReST Values...................42

Impaired Multilingual Sample Compared to the Impaired NA IReST Values.....45

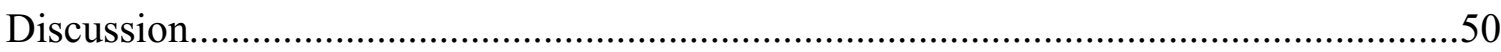

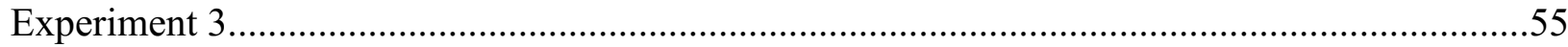

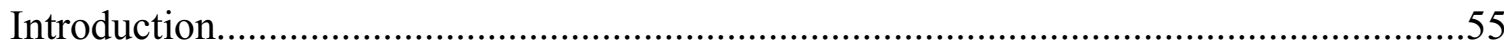

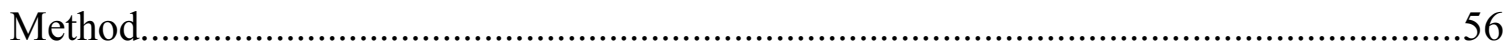

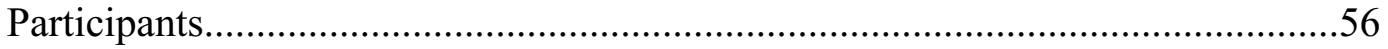

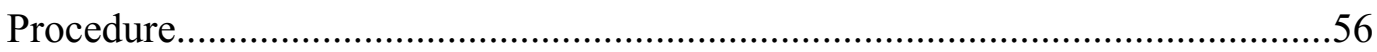

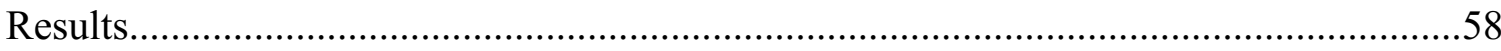

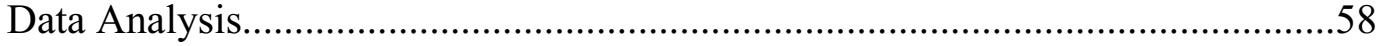

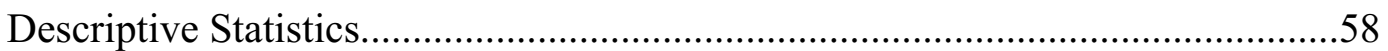

Visual Acuity and Low Vision Simulator Goggles...............................................61

IAG Compared to the Impaired Normative NA IReST Values...............................61

Impaired IAG Compared to the Impaired Normative NA IReST Values..............64

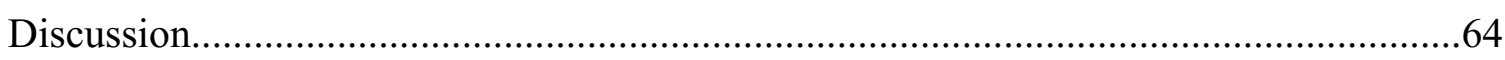

General Discussion ............................................................................................................

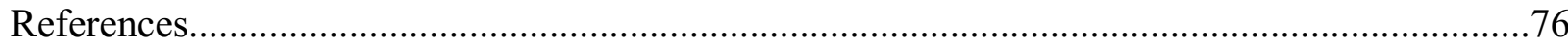

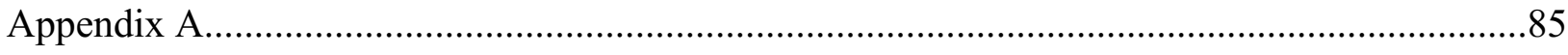

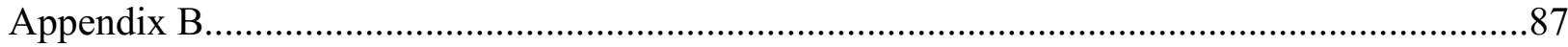

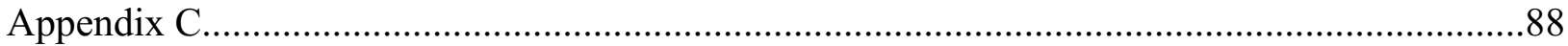

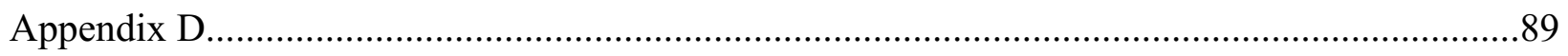




\section{List of Figures}

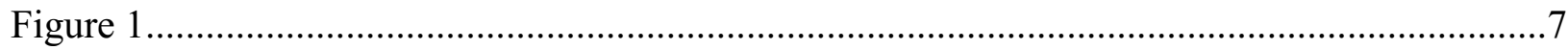

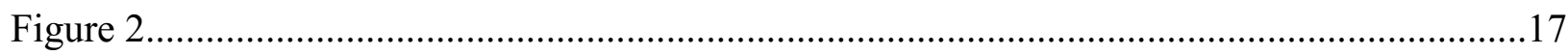

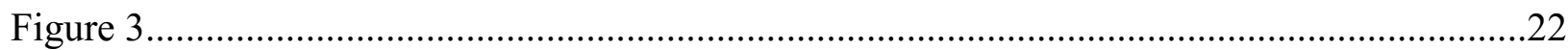

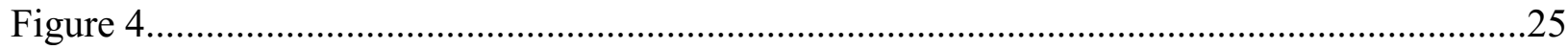

Figure 5

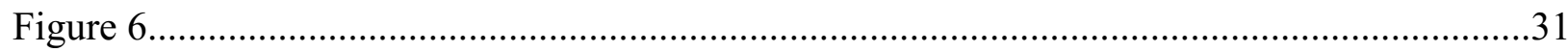

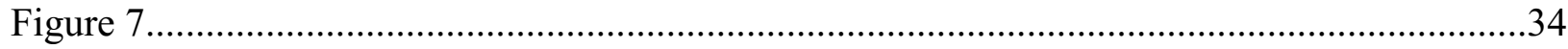

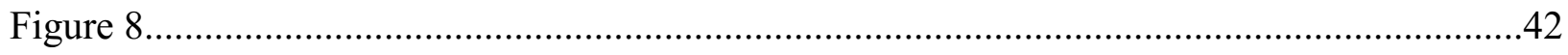

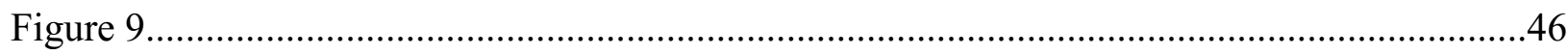

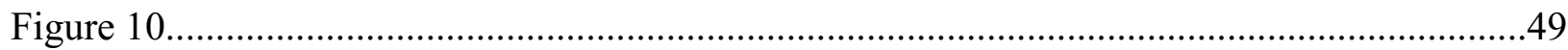

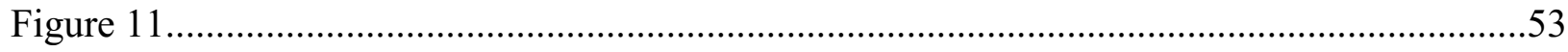

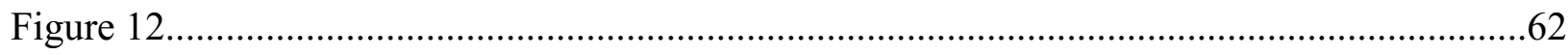

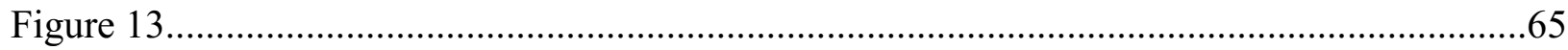

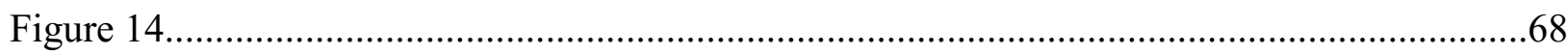

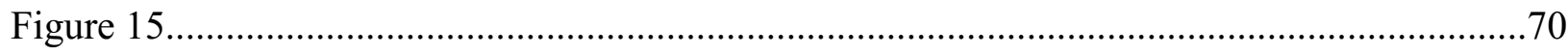




\section{List of Tables}

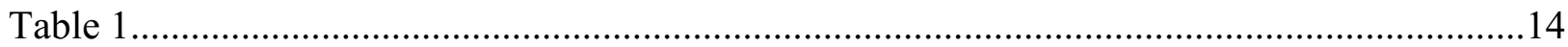

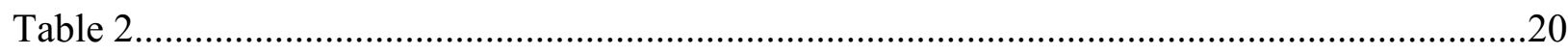

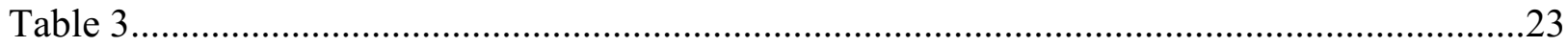

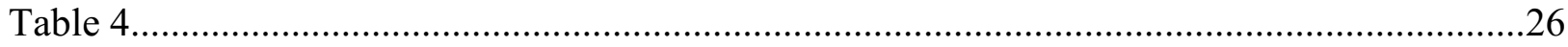

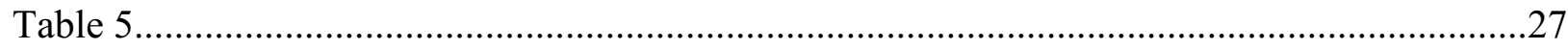

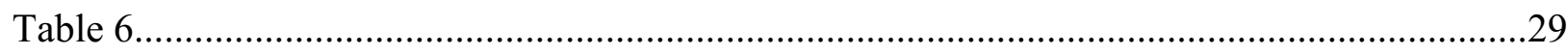

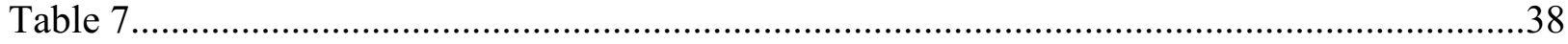

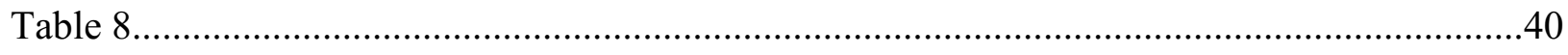

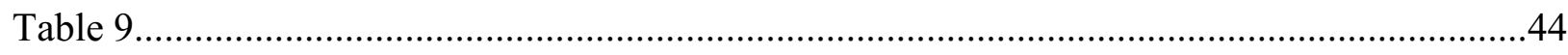

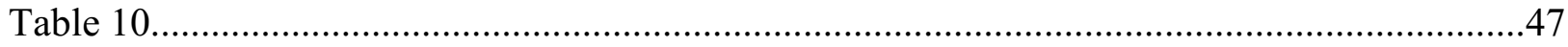

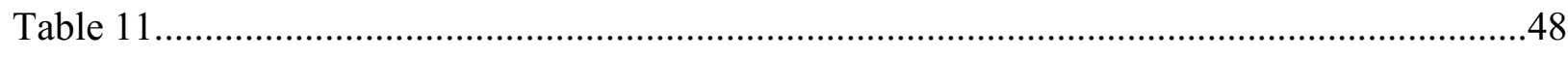

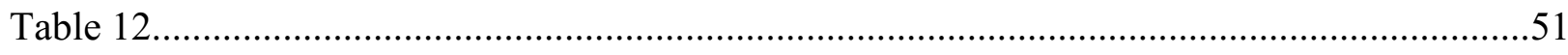

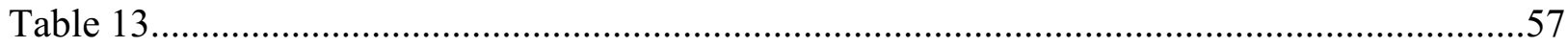

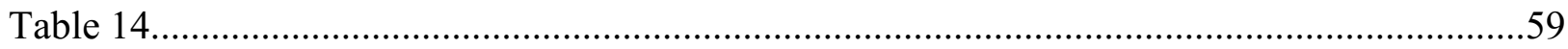

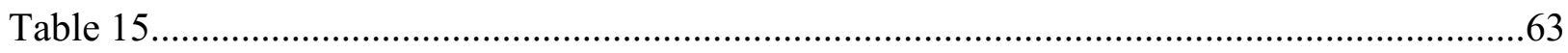

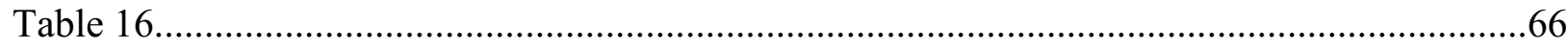

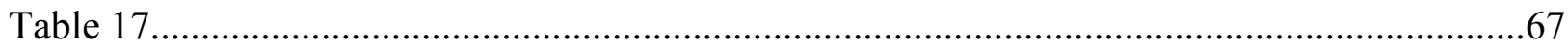

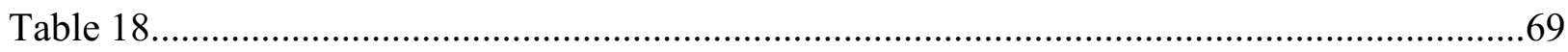




\section{Validation of the International Reading Speed Texts in a Canadian Sample}

The International Reading Speed Texts (IReST) have been developed as a standardized measure used to assess continuous reading in normally sighted individuals, and those with visual impairments (Trauzettel-Klosinski \& Dietz, 2012). Within the IReST, each text contains a measure of mean reading rate (in words per minute) and variance (standard deviation), allowing for comparisons between an individual and the published reading rates. This assessment was developed in Europe, and has been translated and validated in 17 different languages, including English. The English language IReST texts were developed and normalized using a sample in the United Kingdom (UK), and as such, the texts use terms and phrases that are commonly used in

the UK. Although the IReST were developed and normalized in the UK, it is sold internationally, and is used throughout the United States and Canada as a measure to assess reading speed. This raises the problem that because the normative values developed for the IReST are based on a UK English speaking sample, then these normative values may not be valid in a North American (NA) English speaking population. Therefore, the aim of the current thesis is to validate the IReST in an English-speaking NA sample.

\section{Introduction}

\section{Low Vision and Reading Assessments}

There are an estimated 285 million people worldwide who are affected by visual impairments, of which 246 million are diagnosed with low vision (Pascolini \& Mariotti, 2011). Unlike blindness, low vision is defined as a decrease in visual acuity of 20/60 or greater that cannot be corrected through surgery or through corrective lenses (Corn \& Koenig, 1996). Due to declining health, older adults are particularly susceptible to acquiring low vision conditions, with global prevalence rates of low vision in older adults (50+ years old) ranging from $9.5 \%$ to $12.3 \%$ (Bourne et al., 2014; Stevens et al., 2013; Watson, 2001). Visual impairments have been shown to impact quality of life by reducing an individual's ability to complete tasks of daily living such as cooking, driving, recognizing facial expressions, and importantly, reading (BlackmoreWright, Georgeson, \& Anderson, 2013; Rubin, 2013). Of these, researchers have reported that the most frequent reason individuals with sensory impairments seek low vision rehabilitation services is due to difficulty reading (Elliott et al., 1997; Rubin, 2013). Given this, reading is often targeted in low vision rehabilitation through behavioural training (e.g., eccentric viewing training) or through the use of assistive technology devices that magnify text (e.g., Closed 
Circuit Televisions; Massof, 1995; McAllister \& Kammer, 2014; Southall \& Wittich, 2012). Thus, reading speed is one of the most frequently used measures used by researchers and clinicians to assess improvements in clients reading speed pre and post receiving low vision rehabilitation services (Binns et al., 2012; McAllister \& Kammer, 2014; Nguyen, Weismann, \& Trauzettel-Klosinski, 2009; Rubin, 2013; Sass, Legge, \& Lee, 2006).

\section{Factors that Impact Reading Speed}

Reading speed is defined as a measure of the speed at which individual can read a text (McAllister \& Kammer, 2014). Maximum reading speed is the top rate at which a text can be read while still drawing semantic understanding from the text. Typically, reading speed is measured in words per minute (wpm); the minimum reading speed required to fluently read a text at the second grade level is $80 \mathrm{wpm}$, whereas high fluent reading at the $6^{\text {th }}$ grade level requires a minimum reading speed of 160 wpm (Carver, 1992; Whittaker \& Lovie-Kitchin, 1993). There are many factors that influence reading speed, including visual acuity, reading comprehension, and language proficiency.

Visual acuity is a measure of the eye's ability to resolve fine detail at maximum contrast (Attebo, Mitchell, \& Smith, 1996), and can be represented in minutes of arc, the logarithm of minutes of arc (logMAR), or more typically as a quotient where the numerator is the test distance and the denominator indicate the smallest letter that can be read, also known as the Snellen fraction (Attebo et al., 1996; Fosse, 2005; Holladay \& Msee, 2004; Westheimer et al., 1979). The current standard used to assess visual acuity in clinical ophthalmology is the Early Treatment of Diabetic Retinopathy Study (ETDRS) chart; this is a single optotype measure that present strings of 5 letters, on separate lines, with each line decreasing logarithmically in size (Ferris, Kassoff, Bresnick, \& Bailey, 1982). Individuals are asked to read each of the lines out loud, starting from large to small, until they are no longer able to do so. The ETDRS provides clinicians and researchers with a measure of an individual's visual acuity; however, while this measure of visual acuity uses a task similar to reading, it has been shown to be a poor predictor of an individual's reading speed (Ahn \& Legge, 1995; Brussee, van Nispen, \& van Rens, 2014). Nonetheless, visual acuity is an important aspect of reading, and poor visual acuity has been associated with decrease reading speed (Cheong, Lovie-Kitchin, \& Bowers, 2002; Den Brinker \& Bruggeman, 1996; Legge, Rubin, Pelli, \& Schleske, 1985; Rubin, 2013; Virgili \& Acosta, 2006; Whittaker \& Lovie-Kitchin, 1993). 
Although visual acuity is one factor that can significantly impact reading speed, another factor that can influence reading speed is reading comprehension. Reading comprehension and reading speed have a reciprocal relationship, such that each one has been shown to effect the other (Seliger, 1972; Whittaker \& Lovie-Kitchin, 1993). According to a theory called The Simple View of Reading, the task of reading can be divided into two components: decoding and comprehension (Hoover \& Gough, 1990). Decoding refers to efficient word recognition that allows for rapid and accurate access to an individual's vocabulary, and is therefore analogous to reading speed. Comprehension is the ability to draw semantic understanding and interpretation from the text. Both component parts are essential for reading and neither is sufficient on their own (Hoover \& Gough, 1990). Thus, reading comprehension is the ability to draw meaning and understanding from a text, and is assessed through answering questions about the content of the text. Hoover and Gough (1990) examined the relationship between reading speed and reading comprehension and found that limited reading comprehension was associated with decreased reading speed. This relationship may be accounted for through the process of automaticity, whereby individuals with lower levels of reading ability and comprehension do not process the content of a text in an automatic ballistic fashion compared to individuals with higher levels of reading ability and comprehension (Favreau \& Segalowitz, 1983; Segalowitz \& Hulstijn, 2005).

Similar to the impact that visual acuity and reading comprehension have on reading speed, language skill and proficiency have also been shown to impact reading speed; e.g., studies have shown that individuals reading in their first and dominant language (L1) have higher reading speeds than individuals reading in their second language (L2; Cop, Drieghe, \& Duyck, 2015). In general, the weaker-links hypothesis states that monolinguals, individuals who speak one language, have faster reading speeds than bilinguals, those who can speak two languages (Cop et al., 2015; Gollan, Montoya, Cera, \& Sandoval, 2008). The theory posits that due to the decreased frequency of word use and lexical representations of each language, the bilingual speaker will have less practice overall with each language compared to the monolingual speaker and will therefore be less proficient in both languages (Gollan et al., 2008). Alternatively, more recent studies have shown that the decreased language proficiency in bilinguals is only present in their L2 and not in their L1 (Cop et al., 2015). It is therefore important to consider the language background and proficiency of client when assessing reading speed. 


\section{Objective versus Subjective Measures of Reading Speed}

Measures of reading can generally be divided into two categories: self-report and objective measures of reading. Self-report measures of reading ability are generally included in measures of visual functioning, such as the Activities of Daily Living Scale, the Visual Function Questionnaire (VF-14), and the Visual Activities Questionnaire (VAQ), and rely on clients to accurately and honestly report the difficulties they experience when reading (Hart, Chakravarthy, Stevenson, \& Jamison, 1999; Mangione et al., 1992; Sloane, Owsley, \& Bruni, 1992). Each of these measures assess reading in different ways. For example, in the VF-14, individuals must rate the difficulty they experience in reading texts of differing sizes, whereas the VAQ assess difficulty reading under suboptimal conditions, such as poor lighting (Hart et al., 1999; Sloane et al., 1992). Generally, objective measures have been shown to be more accurate predictors of performance compared to self-report measures, are less influenced by cultural and socioeconomic backgrounds, and can be used to provide quantifiable support for evidence-based low vision rehabilitation (Guralnik, Branch, Cummings, \& Curb, 1989; Seiple, Grant, \& Szlyk, 2011; Stelmack, 2001). The discrepancy between clients self-report measures of reading and objective measures of reading ability has been demonstrated by Friedman and colleagues (1999). In their study, Friedman et al. (1999) found that while the majority of participants self-reported reading ability was concordant with their measured reading speeds, $38.2 \%$ of participants showed either strong or mild discordance between their self-reported and measured reading speeds. An example of strong discordance are participants who report no difficulty reading newspaper print, yet are only able to read newspaper print at less than $80 \mathrm{wpm}$. Therefore, while self-report measures provide informative data on individuals perceived reading speed and ability, objective measures of reading speed may be more useful to assess client's actual performance.

When using objective measures of reading speed, researchers will ask individuals to read a short passage of text aloud, while a clinician or researcher follows along to evaluate their performance. Typically, objective measures of reading speed evaluate a person's performance based on their reading speed, which is measured in wpm. Words per minute is calculated as the number of words read correctly (cw), divided by the amount of time taken to read the text in seconds (s), multiplied by 60, i.e., (cw/s) X $60=$ wpm. Generally, objective measures of reading speed also provide researchers and clinicians with measures of clients reading acuity, maximum reading rate, and critical print size (Mansfield, Ahn, Legge, \& Luebker, 1993). Reading acuity, 
similar to visual acuity, is a measure of the smallest print size an individual can read, regardless of the speed at which it can be read. Maximum reading rate is the fastest speed at which an individual can read a text regardless of the print size. Finally, critical print size is the smallest print size an individual can read at their maximum reading rate. While there are many objective measures of reading speed that have been developed, e.g., the Jaeger test, the Bailey-Lovie Near Reading Cards, the Minnesota Low-Vision Reading Test (MNREAD), the Radner Reading Charts, and the International Reading Speed Texts (IReST). Each reading measure has their own strengths and limitations, and each assess different factors associated with reading speed (Bailey \& Lovie, 1980; Legge, Ross, Luebker, \& LaMay, 1989; Radner et al., 1998; Runge, 2000; Trauzettel-Klosinski \& Dietz, 2012).

\section{Measures of Reading Speed}

The oldest known reading assessment is the Jaeger test, it consists of multiple sentence fragments that decrease geometrically in size and clients are asked to read them aloud (Rubin, 2013; Runge, 2000). Originally printed in German, the Jaeger test was translated into English and French, however, due to the changes in font typeface that occurred in the translations, this caused the loss of Jaegers geometric size progression (Runge, 2000). The development of the Bailey-Lovie cards addressed this issue by having the standard print size of the texts decrease in size logarithmically (Bailey \& Lovie, 1980). The administrator of the test asks individuals to read texts with two to six unrelated words; however, it has been shown that assessing reading speed using random words is a poor predictor of reading speed when compared to reading full sentences in both visually impaired and normally sighted individuals (Fine \& Peli, 1996; Fine, Rubin, Hazel, \& Petre, 1999). Given this, rather than being used to assess reading speed, the Bailey-Lovie cards are now used by clinicians to assess the level of magnification individuals with low vision require to read a normal sized text (e.g., Times New Roman, 12 point font; Rubin, 2013).

In an attempt to provide a reading speed measure under more naturalistic reading conditions, both the MNREAD and Radner acuity charts adopted the Bailey-Lovie cards logarithmic progression. However, rather than using sentence fragments to assess reading speed, they used coherent sentences (Legge et al., 1989; Mansfield et al., 1993; Radner et al., 1998). The MNREAD consists of a series of 19 short, simple sentences of 60 characters. Each sentence is written at a $3^{\text {rd }}$-grade reading level, each is presented on three lines of texts, and each sentence 
decreases logarithmically in size (see Figure 1; Brussee et al., 2014). The Radner acuity charts are similar to the MNREAD, but were originally developed in German and have since been translated into 11 different languages including English (Brussee, van Nispen, Klerkx, Knol, \& van Rens, 2015; Brussee et al., 2014). The Radner acuity charts consist 24 different sentences of 14 words each, that decrease logarithmically in size and are written at a $3^{\text {rd }}$ to $4^{\text {th }}$ grade reading level (see Figure 1; Brussee et al., 2015, 2014; Radner et al., 1998). Finally, similar to the MNREAD, the Radner charts can also be used to determine a client's reading acuity, maximum reading rate, and critical print size.

While the MNREAD and Radner charts have multiple strengths (for example, standardized for reading level and difficulty; sentences decrease in size logarithmically; available in different languages; quick to administer), they also have limitations. While their sentences are short and easy to comprehend, the MNREAD has 10-14 words per sentence and Radner has 14 words per sentence, these short sentences may actually overestimate reading speed of prolonged reading (Altpeter, Marx, Nguyen, Naumann, \& Trauzettel-Klosinski, 2015). Therefore, using a reading speed measure that uses short, simple sentences, compared to continuous sentences (i.e., a paragraph), may not provide researchers and rehabilitation clinicians with accurate measures of reading speed. A recent study by Altpeter, Marx, Nguyen, Naumann, and Trauzettel-Klosinski (2015) found that assessing reading speed using continuous sentences resulted in lower variability across multiple texts compared to when using single sentence measures of reading speed. While the MNREAD and Radner charts are useful measures and provide researchers and clinicians with relevant information, e.g., reading acuity, maximum reading rate, and critical print size, they are limited in their ability to measure reading speed due to this overestimation.

\section{The International Reading Speed Texts (IReST)}

The IReST was developed to address some of the limitations of the previously mentioned measures of reading speed through the use of continuous sentence paragraphs (Hahn et al., 2006; Trauzettel-Klosinski \& Dietz, 2012). The IReST consists of a set of 10 paragraphs that have been standardized for the number of words per text ( $M=132$ words), text difficulty, sentence complexity, and syntax (see Figure 1; Trauzettel-Klosinski \& Dietz, 2012). Each of the texts is based on an encyclopaedia entry, and was adapted so that it can be read at a $6^{\text {th }}$ grade reading level. The IReST were specifically developed as a standardized measure of reading speed that allows for repeated measurements across multiple languages; each of the 10 texts have been 


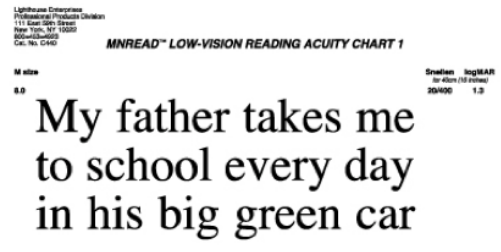

Everyone wanted to

" go outside when the m rain finally stopped

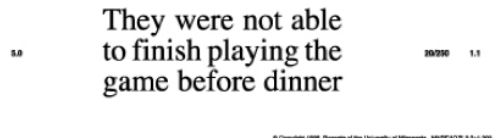

A.

C.

\begin{abstract}
In a small town a greengrocer had opened a shop that was located above a deep cellar. Every night, mice came in droves out of this cellar into the shop. They ate apples and pears, grapes and nuts and did not spare the vegetables and potatoes either. No goods that were in the shop were safe from the small intrusive rodents between midnight and sunrise. As long as there was noise in the streets at night and cars were driving by, the mice still stayed quietly in the cellar. But as soon as the old clock on the town hall had struck midnight and it became quiet in the street, they came out in droves, enjoyed the sweet fruits and celebrated real feasts, whose remains filled the owner with despair every morning when he entered the shop. So he tried to protect himself against the mice. At first he set up traps all over the shop.
\end{abstract}

B.
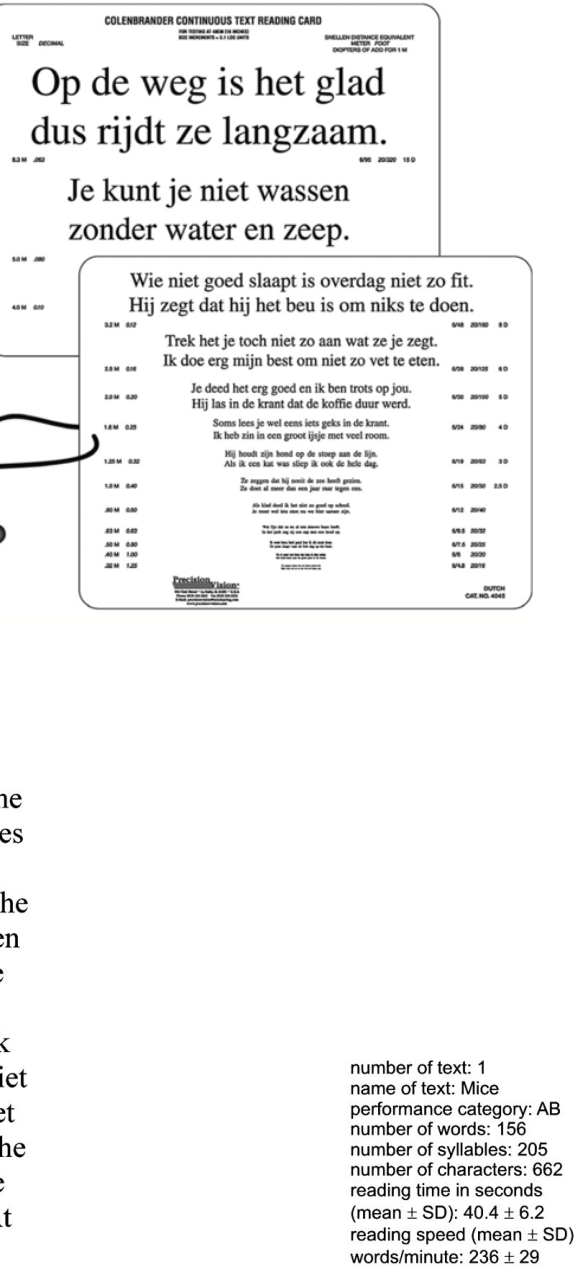

Figure 1. Traditional measures of reading speed: the MNREAD (A), the Radner acuity charts (B), and the IReST (C). 
translated into 17 different languages, e.g., English, French, Japanese, German, and there are normative values, e.g., mean wpm and standard deviation, for each text, in each language. The IReST were originally validated on a sample of 436 participants between the ages of 18-35 years old (25 participants per language, 36 participants for the Japanese texts). In addition, they can be used to assess reading speed for individuals with and without visual impairments (TrauzettelKlosinski \& Dietz, 2012).

To assess reading speed, individuals are instructed to hold one of the IReST at a $40 \mathrm{~cm}$ distance and are instructed to read the entire text out loud, as quickly as possible and without going back and making corrections. As the IReST consists of 10 comparable texts, reading speed can theoretically be assessed while eliminating practice effects, which allows for a more accurate measure of reading speed. Practically, the IReST can be used to assess reading speed in individuals with low vision before and after receiving low vision rehabilitation to determine the outcome of rehabilitation, or they can be used to determine how reading speed changes as a function of the type of assistive reading device that is used, e.g., handheld magnifier vs. CCTV (Nguyen et al., 2009).

Although the IReST does address some of the limitations of other measures of reading speed, such as the MNREAD and Radner acuity charts, they do have their own limitations. Despite the fact that a continuous sentence format of the IReST allows clinicians to determine clients maximum reading rate, the absence of a logarithmic progression in text sizes means that a client's critical print size and reading acuity cannot be determined. Further, and important to the current thesis, while each of the different language versions of the IReST were validated in their country of origin, they have not since been revalidated and have not been validated in other English speaking countries. This is particularly problematic for the English language IReST as they were originally developed and validated in the United Kingdom (UK), and as such they use sentence structure, grammar, syntax, terms, and phrases that may be unfamiliar to English language speakers outside of the UK. For example, one of the words used in one of the texts is "greengrocer," however, in 2008 the prevalence of the word "shopkeeper" was 7X greater than the use of the word "greengrocer" (Google Ngram Viewer, n.d.). Therefore, the normative data for the English language IReST may not be a valid measure of reading speed for English speaking North Americans. While the IReST were developed to assess reading speed and ability in both normally sighted and low vision clients, normative values have thus far only been 
developed for normally sighted individuals. Thus, there are no normative values for use with individuals who are affect by visual impairments. Additionally, due to the nature of the assessment, i.e., longer text paragraphs, there may be increased change of error in test administration when using the IReST compared to the MNREAD or Radner charts. For example, not following the standardized IReST administration protocol, e.g., telling clients to read the texts as quickly as possible without going back and making corrections, may impact the measured reading speed (Lezak, Howieson, Bigler, \& Tranel, 2012). Finally, while the IReST addresses the limitations of some other measures of reading speed through the use of continues sentence paragraphs, they do not assess reading comprehension; as previously mentioned, reading comprehension has been shown to significantly impact reading speed (Hoover \& Gough, 1990) and it is conceivable that participants may read the text quickly but not understand its content, e.g., Palmer, MacLeod, Hunt, \& Davidson (1985), thereby overestimating reading speed. Consequently, the inclusion of reading comprehension questions in the IReST could increase the reliability of the measure.

\section{Purpose of the Current Thesis}

The purpose of the current thesis is to validate the English language IReST in a NA sample. As previously mentioned, the English Language IReST were developed in the UK and, therefore, the normative data that was derived from a UK sample may not be valid in a NA English speaking population. By validating the measure in a NA sample, the present study will provide new normative values for English speaking North Americans on the IReST. The aim of this thesis is also to determine the impact of simulated visual impairments on the IReST by administering the test to individuals with artificially impaired acuity of 20/80 through filtered glasses. In doing so, this may provide researchers and clinicians with normative data that can be used as a guide when assessing reading speeds in low vision clients. As the data will be collected in Montreal, Quebec, this will also provide the opportunity to examine the impact of bilingualism on reading speed with the IReST. Due to the nature of the assessment, i.e., continuous sentence paragraphs, the improper administration the IReST by not following the protocol or standardization procedures may result in measurement errors. This aspect will also be examined by administering the IReST to different samples with and without following the administration protocols; i.e., administering the task by asking participants to read the text aloud as quickly as 
possible and without going back and making corrections, compared to simply asking clients to read the text aloud.

The research questions to be addressed in this thesis are: (1) are the normative values of the English language IReST valid in a NA sample, and, if not, what are the normative values for this population; (2) what is the impact of simulated reduced acuity on the English language IReST, and what are the normative values for this population; (3) what is the impact of bilingualism on reading speed on English language IReST; and finally, (4) are there significant differences in the measurement of reading speed on the English language IReST when test administrators do not follow the administration protocols. It is hypothesized that (1) the NA English language IReST values will fall below the standards established by the UK English language IReST texts; (2) individuals with simulated visual impairments will read the texts significantly slower then individuals with normal or corrected to normal vision; (3) a multilingual sample of English speaking North Americans will read the texts significantly slower than those whose first and dominant language is English; and finally, (4) that not following administration protocols will significantly impact the results of the assessment. 


\section{Experiment 1}

The first experiment will determine whether the normative values of the IReST are valid in an English-speaking NA sample. The English language IReST were originally developed in the UK, and use sentence structure, grammar, syntax, terms, and phrases that may be less familiar to NA English speakers, which may impact their reading speed on these measures. It is therefore hypothesized that this sample of NA English speakers would show significantly slower reading speeds (i.e., reduced wpm) when compared to the normative IReST values provided by the original IReST study (Trauzettel-Klosinski \& Dietz, 2012).

To my knowledge, there are no studies that have examined the differences in reading speeds between English NA and UK samples. However, there are studies that have examined differences in articulation speed between English speaking NA and UK samples (Jacewicz, Fox, O’Neill, \& Salmons, 2009; Whiteside, 1996). For example, Jacewicz et al. (2009) compared the differences in articulation speed between North Americans living in Wisconsin and North Carolina, when participants were asked to read aloud from a text. They found there were significant differences between English speaking Wisconsinites (3.54 syllables/second) and North Carolinians (3.27 syllables/second). Similarly, Whiteside (1996) examined the differences in articulation speed between Northern British English speaking men and women, and found that there were significant differences in articulation speed between men (4.10 syllables/second) and women (3.38 syllables/second). While Jacewicz et al. (2009) and Whiteside (1996) only assessed articulation speed, these studies illustrate that there are regional differences in the way language is processed and therefore may provide evidence to indicate there may also be regional differences in reading speed.

One factor that influences reading speed is language skill and proficiency (Cop et al., 2015; Gollan et al., 2008). As data for this study was collected in Montreal, a high proportion of the city's population is bilingual, or even trilingual, participants in this study were restricted to individuals whose first and dominant language (L1) was English (Statistics Canada, 2012). Studies have shown that there are significant differences in reading speeds between participants when asked to read a text in their L1 versus their L2 and this has been attributed to discrepancies in language proficiency and automaticity in these languages (Cop et al., 2015; Favreau \& Segalowitz, 1983; Gollan et al., 2008; Lee \& Schallert, 1997; Segalowitz \& Hulstijn, 2005). 
Thus, to remove this variability, only participants who's L1 was English were included in this study.

Finally, while the IReST texts were developed to assess reading speed in individuals with sensory impairments, they have not to my knowledge been validated on this population (Trauzettel-Klosinski \& Dietz, 2012). This may be due to the difficulty in developing normative values for this heterogeneous population; individuals with sensory impairments have varying degrees of visual acuity depending on the severity of their condition and they have varying levels of reading ability depending on how well they have adapted to their condition (Crossland, Culham, \& Rubin, 2004; Nguyen, Stockum, Hahn, \& Trauzettel-Klosinski, 2011). Therefore, the impact of sensory impairments on reading speed will be assessed in this experiment through the use of a simulated reduction in visual acuity. A simulated visual impairment can be used to reduce a participant's visual and contrast sensitivity to simulate low vision (Elliott, Bullimore, Patla, \& Whitaker, 1996; Gao \& Loomes, 2016; Rousek \& Hallbeck, 2011). One of the advantages of a simulated visual impairment is that this will result in a uniform reduction in visual acuity and contrast sensitivity across participants to determine the general impact of low visual acuity (e.g., 20/80). Conversely, a limitations of using a simulated visual impairment is that this may result in an underestimate of this populations true reading speed as individuals with low vision typically have adapted to their impairments or have developed compensatory techniques to increase their reading speed, e.g., through the use of a preferred retinal locus (Crossland et al., 2004; Nguyen et al., 2011). Using a simulated visual impairment does not take these compensatory strategies into account, as normally sighted individuals will not have had enough time to adapt to the simulation of visual impairment.

This experiment will; (1) determine whether the normative values of the IReST texts are valid in an English-speaking NA sample and (2) determine the impact of a simulated sensory impairment on reading speed on the IReST. In this experiment normally sighted, L1 English speaker were asked to read all 10 English language IReST texts following the IReST administration protocols. Participants read half of the texts with their normal, or corrected to normal vision, and half of the texts were read using a simulated 20/80 visual impairment. After reading each text, participants were asked a multiple choice reading comprehension question to ensure they understood the content of the text they read. It was hypothesized that; (1) the NA English language IReST values would fall outside of the standards established by the UK 
English language IReST texts (Trauzettel-Klosinski \& Dietz, 2012) and (2) individuals with simulated visual impairments would read the texts significantly slower then individuals with normal or corrected to normal vision.

\section{Method}

The research protocol was approved by the research ethics board of Concordia University (certificate 30003975), in accordance with the Canadian Tri-Council Policy Statement of ethical conduct for research involving humans.

\section{Participants}

Fifty undergraduate students $(n=50)$ between the ages of 18 and $45(M=24.06, S D=6.45)$ were recruited from Concordia University to participate in this study. Participants were recruited using the Concordia University Psychology Participant Pool and were granted course credit for participating in this study. The inclusion criteria were; (1) participants were required to have normal, or corrected-to-normal, vision; and (2) participants first and dominant language (L1) was English. For participant demographics, please see Table 1.

\section{Measures}

Concordia University Language Background Questionnaire. The Concordia University Language Background Questionnaire (see Appendix A) was used to obtain participants' basic demographic information and to assess their language background. Data were collected on participants' first language, second language, third language (if any), and dominant language, as well as information on participant's language of instruction throughout their primary, secondary, and post-secondary educations. Participants were also asked to rate their self-reported speaking, reading, writing, and listening ability in English, French, and other known languages. Finally, participants were then asked whether or not they had any visual and/or hearing impairments, and whether they had any known reading or attention disabilities.

Reading Speed. Participants were asked to read the IReST aloud as the researcher followed along on a printed copy of the text to record any errors that were made. Reading speed was measured using a stopwatch that was started when participant read the first word of each text, and was stopped when the read the last word of each text. Reading speed, in words per minute (wpm), was calculated using the formula (cw/s) X $60=\mathrm{wpm}$, where $\mathrm{cw}$ is the number of words read correctly and $s$ is the amount of time it took participants to read the text in seconds. 
Table 1.

Participant demographics

\begin{tabular}{lcc}
\hline Demographics & $n$ & $\%$ \\
\hline Total Survey Respondents & 50 & $100 \%$ \\
Male & 8 & $16 \%$ \\
$\quad$ Female & 42 & $84 \%$ \\
Age & & \\
$18-25$ & 43 & $86 \%$ \\
$26-35$ & 2 & $4 \%$ \\
$36-45$ & 5 & $10 \%$ \\
Visual impairment not corrected through lenses & & \\
Yes & 0 & $0 \%$ \\
No & 50 & $100 \%$ \\
Known hearing impairment & & $0 \%$ \\
Yes & 0 & $100 \%$ \\
No & 50 & \\
Reading/Attention Disability & & $12 \%$ \\
Yes & $58 \%$ \\
No & 64 & $88 \%$ \\
\hline
\end{tabular}


Freiburg Visual Acuity and Contrast Sensitivity Test (FrACT). The FrACT is a computer-based measure developed to assess visual acuity and contrast sensitivity (Bach, 1996). The FrACT has been shown to be a valid and reliable measure of visual acuity in comparison to the current gold standard measure used by researchers and clinicians, the ETDRS charts (Kurtenbach, Langrová, Messias, Zrenner, \& Jägle, 2013). The FrACT has been shown to have adequate test-retest reliability (Schulze-Bonsel, Feltgen, Burau, Hansen, \& Bach, 2006). The FrACT was presented to participants on an Apple iMac (21 inch - 2015 model) at a seated distance of $140 \mathrm{~cm}$, and participants were asked to complete both measures of visual acuity and contrast sensitivity. The visual acuity task consists of 24 trials in which participants are presented with Landolt C's at various sizes and orientations; participants were asked to press the arrow key that corresponded to the orientation of the gap in the "C" (Up, Down, Left, or Right). The more accurate the participant was, the smaller the "C" would get. The contrast sensitivity task consists of 24 trials and followed the same stimulus presentation paradigm; however, rather than decreasing in size with increased accuracy, the contrast between the Landolt $\mathrm{C}$ and the background decreased such that it became more difficult to discriminate the background from the foreground. The FrACT was used to assess visual acuity and contrast sensitivity at three time points throughout the experiment: once at baseline for normal vision condition and then before and after reading the texts in the simulated low vision condition.

\section{Materials}

International Reading Speed Texts (IReST). The IReST are standardized reading materials used to assess reading speed under naturalistic conditions (i.e., newspaper print; Trauzettel-Klosinski \& Dietz, 2012). In this study, participants read all 10 English language IReST texts. The order in which participants read the texts was counter-balanced to account for learning effects and fatigue. Each text was printed in 10 point Times New Roman font, on white cardstock with $98 \%$ contrast, and was presented left justified, in the center of the page, in portrait orientation. In the normal vision condition, the texts were presented to participants at a distance of $40 \mathrm{~cm}$, whereas in the simulated impairment condition participants were permitted to hold the texts as close as required to facilitate reading per the IReST administration protocol for assessing individual with visual impairments. Following the administration protocol, participants were instructed to read the text aloud as quickly as possible, without going back and making corrections. 
Fork in the Road Goggles (20/80). Low vision simulation goggles were used to simulate a visual impairment of 20/80 (or $0.6 \log$ MAR) in the impaired vision condition. In the normal vision condition, participants wore the low vision simulator goggles with the lenses removed to control for differences in the reading task across conditions. The goggles were purchased from Fork in the Road Simulation Goggles (Vision Rehabilitation Services LLC, www.lowvisionsi mulators.com). Previous studies have used these simulator goggles to study, for example, ways to improve signage and navigation in health care settings, and they have been used to assess way in which digital displays can be adapted to be more user friendly for individuals with low vision (Gao \& Loomes, 2016; Rousek \& Hallbeck, 2011). A limitation in these studies, however, was that the researchers did not assess participant's baseline visual acuity, nor did they assess the degree to which the low vision simulators were successful in simulating a visual impairment. Therefore, to assess whether the simulator goggles are effective in simulating a 20/80 visual impairment, participants' visual acuity was assessed throughout the experiment using the FrACT.

\section{Procedure}

Upon arrival, participants were instructed on the content and procedure of the study and informed consent was obtained. Participants then completed the Concordia University Language Background Questionnaire and any remaining questions concerning the study were answered. Participants were systematically assigned to a counterbalanced condition and were asked to read all 10 IReST aloud. The normal and impaired vision conditions were counterbalanced such that the first set of 5 IReST were read with either the participants normal/corrected-to-normal vision or with the simulated $20 / 80$ visual impairment using the low vision simulator goggles. The order in which the IReST were read was also counterbalanced to reduce learning effects and fatigue.

In the normal vision condition, participants wore the goggles with the low vision simulator lenses removed and baseline measures of participant's visual acuity and contrast sensitivity were obtained using the FrACT (see Figure 2). Following this, 5 of the IReST were presented to the participants at a distance of $40 \mathrm{cms}$, and they were asked to read each text aloud as quickly as possible and without going back and making corrections. After reading each text participants were asked a short multiple choice reading comprehension question pertaining to the text that they had just read. Once participants finished reading the 5 IReST with their normal/corrected-to-normal vision, they completed the simulated visual impairment condition. 


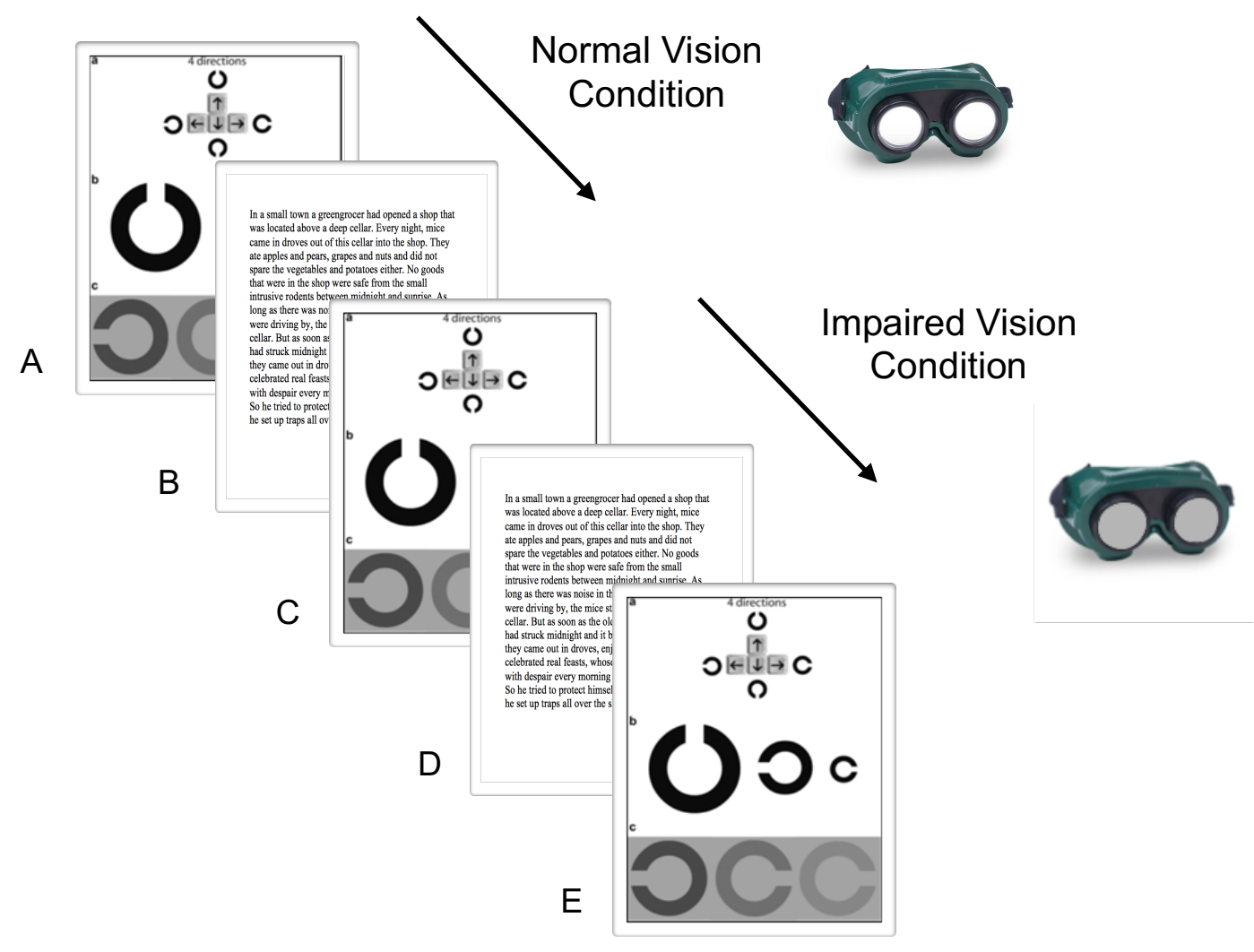

Figure 2. Experimental paradigm. (A) In the normal vision condition, participant's baseline measures of visual acuity and contrast sensitivity while wearing placebo goggles were measured using the FrACT. (B) Participants the read five of the IReSTs while wearing the placebo goggles. (C) In the simulated impairment condition, participants wore the 20/80 low vision simulator goggles, and their visual acuity and contrast sensitivity were measure to ensure the goggles were operating effectively. (D) Participants then read the five remaining IReSTs while wearing the low vision simulator goggles. (E) Final measures of participant's visual acuity and contrast sensitivity were obtained to ensure that they did not adapt to the goggles. If participants started in the Impaired vision condition, then the procedure was revered. 
Participants were given the 20/80 low vision simulator goggles, and were asked to read aloud a practice text to adapt to the low vision simulators. Once they finished reading the practice text, another measure of participant's visual acuity and contrast sensitivity was obtained to ensure that the low vision simulator goggles were operating effectively. Participants were then instructed to read the remaining 5 IReST aloud as quickly as possible and without going back and making corrections. Again, after reading each text participants were asked a multiple-choice reading comprehension question. In this condition participants were permitted to hold the texts as close as required to facilitate reading, as stipulated in the IReST administration protocol for assessing individuals with visual impairments, and the distance at which each text was read was recorded using a tape measure. Once a participant finished reading the remaining IReST, another measure of their visual acuity and contrast sensitivity was obtained to ensure that participants did not adapt to the simulator goggles; i.e., to ensure participants experienced the same degree of visual impairment throughout the simulated visual impairment condition. If participants completed the visual impairment condition first, the same procedure was followed in reverse.

\section{Results}

\section{Data Analysis}

As there were 50 participants in the experiment, each reading 5 texts in the normal condition and 5 texts in the impaired condition, the data were combined to create 25 metaparticipants in each condition to analyze the results. In order to only examine the variance attributable to differences in reading speed caused by the IReST texts themselves participant's between-subject variability, their natural between-subject differences in reading speed, were removed. This was done following the procedure established by Loftus and Masson (1994); that is, subtracting the difference between the subjects mean and the grand mean from each of the participants scores. Between-subject variability was removed from both the impaired and normal vision conditions.

To determine whether the normative values of the IReST texts are valid in an Englishspeaking NA sample, difference scores (in wpm) between the NA sample and normative data provided by the IReST were examined using a two-sample dependent (or paired) t-test (with Holm-Bonferonni correction for multiple comparisons). The $95 \%$ confidence intervals around the mean difference were used as measure of the margin of uncertainty around the estimated 
difference between the two means, and bias-corrected Hedge's $g\left(g^{*}\right)$ as a measure of effect size (Kline, 2013).

A Bayesian two-sample dependent (or paired) t-test was used to determine: (1) whether the low vision simulator goggles impaired participant's visual acuity and contrast sensitivity to the same degree throughout the experiment; and (2) to determine whether there were significant differences between the simulated visual impairment condition and the normal vision condition. A Bayesian analysis was used due to known issues with traditional null hypothesis significance testing (NHST; Dienes, 2011; Kline, 2013; Wetzels et al., 2011). Further, Bayes analysis allows for the interpretation of null results as either insufficient evidence to make a conclusion, or as finding evidence for the null (Dienes, 2011). Bayes factors (BF) were calculated using JASP 0.8.0.1 (JASP Team, 2016) and for each analysis a Cauchy prior width of 0.707 was used. A $\mathrm{BF}_{10}$ is the strength of evidence for the research hypothesis, and the $\mathrm{BF}_{01}$ is the strength of evidence for the null hypothesis. For each analysis, the robustness of the posterior was checked using wider priors of 1.00 and 1.50 to ensure that the choice of prior distribution did not significantly impact the results. As per the recommendations of Kruschke (2014) and Wetzels et al. (2011), the results in this thesis are reported as both traditional NHST and Bayesian twosample dependent t-tests, along with measures of effect sizes and confidence intervals, as there are presently no standards established in the field for reporting the results of a Bayesian analysis. Therefore the reader may interpret their statistic of choice.

\section{Descriptive Statistics}

All participants' first and dominant language was English, with 90\% of participants reporting that they were fluent English language readers and 10\% reporting that they were very good English language readers. All participants (100\%) were bilingual, and 54\% of participants were trilingual. See Table 2 for a breakdown of participants self-reported language fluency (speaking, reading, writing, and listening ability) in English, French, and other third languages.

To ensure that participants understood the context of each text that they read, as opposed to simply reading the text as quickly as possible without retaining any content, participants were asked to answer a reading comprehension questions about each text after it was read. In the normal vision condition, $88 \%$ of the questions that were asked were answered correctly, and in the simulated impairment condition $86 \%$ of the questions were answered correctly. See Appendix B for a breakdown of the proportion of questions answered correctly for each of the 
Table 2.

Language Fluency

\begin{tabular}{lcccccc}
\hline & $n$ & No Ability & Elementary & Moderate & Very Good & Fluent \\
\hline English & 50 & & & & & \\
Speaking & & 0 & 0 & 0 & 3 & 47 \\
Reading & 0 & 0 & 0 & 5 & 45 \\
Writing & 0 & 0 & 1 & 7 & 42 \\
Listening & 0 & 0 & 0 & 2 & 48
\end{tabular}

French $\quad 50$

$\begin{array}{lccccc}\text { Speaking } & 2 & 8 & 17 & 16 & 7 \\ \text { Reading } & 1 & 4 & 12 & 24 & 9 \\ \text { Writing } & 5 & 11 & 18 & 10 & 6 \\ \text { Listening } & 3 & 5 & 8 & 18 & 16\end{array}$

Other 27

Speaking

27

Reading

Writing

Listening

$\begin{array}{llcll}0 & 3 & 17 & 4 & 3 \\ 7 & 4 & 7 & 6 & 3 \\ 7 & 9 & 7 & 3 & 1 \\ 1 & 3 & 10 & 5 & 8\end{array}$

Note. Of the 27 participants with third languages, these languages included: Arabic, Dutch, Farsi, French, German, Hebrew, Hindu, Italian, Moroccan, Punjabi, Spanish, Tagalog, and Tamil 
texts. It appeared that participants had difficulty answering the reading comprehension question for text 2 , however, it is believed that this difficulty reflects the poor quality of content of the question as opposed to participants not understanding the content of the text.

\section{Visual Acuity and Low Vision Simulator Goggles}

Participants' visual acuity and contrast sensitivity were measures at three time points: (1) at baseline without the simulator goggles in the normal vision condition, (2) before reading the texts with the simulator goggles in the impaired condition, and (3) after reading the texts with the simulator goggles in the impaired condition. At the first time-point, participants had normal, or corrected to normal, vision, with an average visual acuity of $-0.11 \log$ MAR $(M=-0.11, S D=0.09$, $C I[-0.13,-.09])$ and an average contrast sensitivity of $2.01 \log C S(M=2.01, S D=0.21, C I[1.95$, 2.06]), see Figure 3. The low vision simulator goggles failed at both the second and third timepoints at simulating a 20/80 visual impairment, however, they were successful at inducing a simulated visual impairment of 20/60, or $0.50 \log$ MAR, which is the threshold in Quebec to be eligible for low vision services in Quebec (RAMQ, 2006). At the second time-point the goggles simulated a visual acuity of $0.51 \log \operatorname{MAR}(M=0.51, S D=0.16, C I[0.46,0.55])$ and contrast sensitivity of $0.93 \log C S(M=0.93, S D=0.28, C I[0.85,1.01])$, and at the third time-point the goggles simulated a visual acuity of $0.49 \log \operatorname{Mar}(M=0.49, S D=0.17, C I[0.44,0.54])$ and contrast sensitivity of $0.96 \log C S(M=0.96, S D=0.18, C I[0.91,1.01])$. There were no significant differences between the participants visual acuity at the second and third time-point, $t(49)=0.73$, $p=0.47, d=0.10, C I[-0.03,0.07]$; further Bayesian analysis revealed a $\mathrm{BF}_{01}$ factor of 5.05, indicating that the probability of the null hypothesis is 5.05 times greater than the probability of the research hypothesis. There were also no significant differences between the participants contrast sensitivity at the second and third time points, $t(49)=-0.81, p=0.44, d=-0.11, C I[-0.11$, 0.05 ]; further, Bayesian analysis revealed a $\mathrm{BF}_{01}$ factor of 4.78 , indicating that the probability of the null hypothesis is 4.78 times greater than the probability of the research hypothesis. Therefore, participants received the same degree of simulated visual impairment across the impairment condition.

\section{NA Sample Compared to Normative IReST Data}

To test the hypothesis that NA English IReST values would fall outside of the standards established by the UK English IReST texts, mean reading speeds in wpm were calculated for each text in the normal vision condition (see Table 3). Multiple two-sample dependent t-tests 

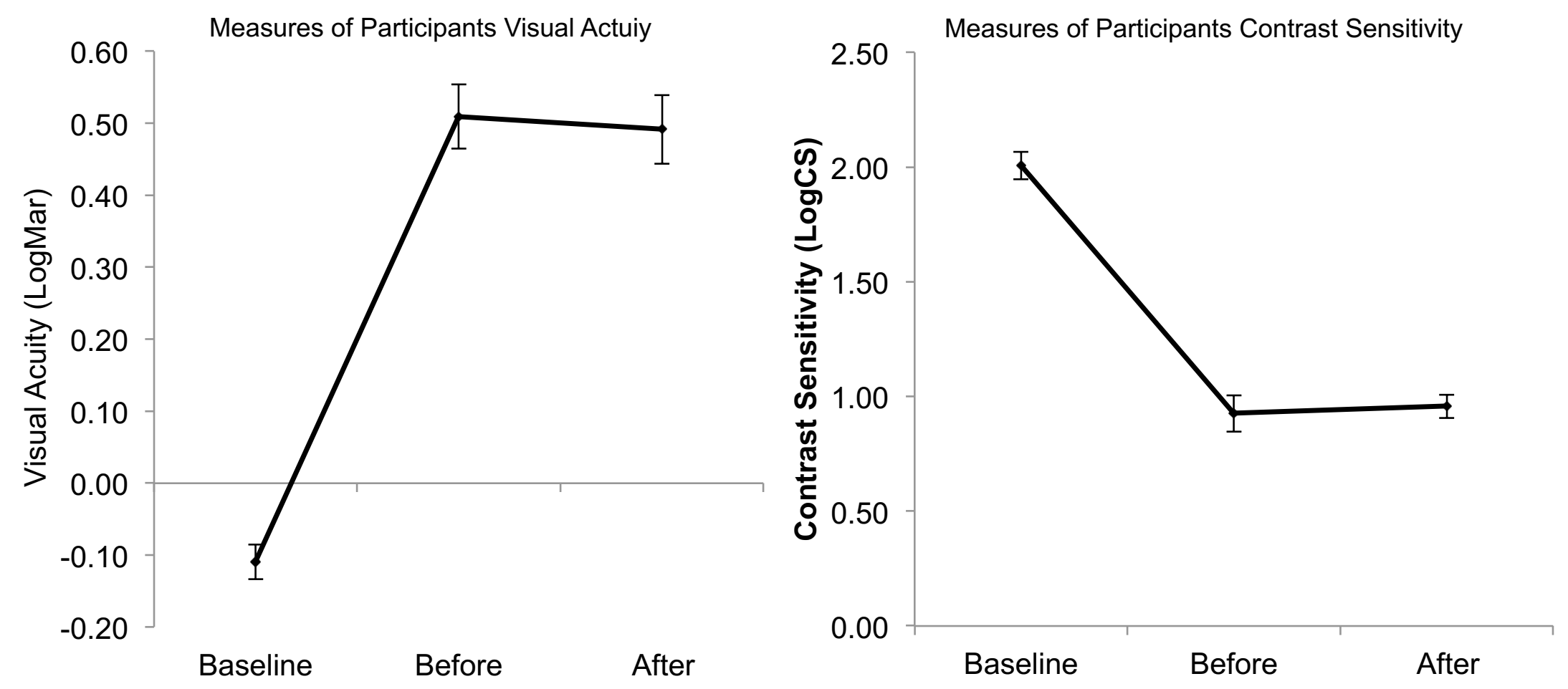

Figure 3. Measures of participant's visual acuity and contrast sensitivity. These figures represent changes in participant's visual acuity and contrast sensitivity from baseline to before and after the simulated visual impairment conditions. Error bars represent $95 \%$ confidence intervals around the mean. 
Table 3.

Results of the Two Sample Dependent t-test Between Reading Speeds on the IReST of UK and NA English Samples.

\begin{tabular}{|c|c|c|c|c|c|c|c|c|c|c|c|c|}
\hline & \multirow[b]{2}{*}{$\mathrm{n}$} & \multicolumn{2}{|c|}{ UK Sample } & \multicolumn{2}{|c|}{ NA Sample } & \multicolumn{7}{|c|}{ Dependent Samples t-test } \\
\hline & & $m$ & $s d$ & $m$ & $s d$ & Mdiff & $d f$ & $t$ & $p$ & $C I$ & Hedge's g* & $C I$ \\
\hline IReST 1 & 25 & 236 & 29 & 205.95 & 13.00 & 30.05 & 48 & 4.73 & $<.001$ & {$[17.26,42.83]$} & 1.32 & {$[0.70,1.93]$} \\
\hline IReST 2 & 25 & 243 & 31 & 213.85 & 10.43 & 29.15 & 48 & 4.46 & $<.001$ & {$[15.99,42.30]$} & 1.24 & {$[0.63,1.85]$} \\
\hline IReST 3 & 25 & 227 & 29 & 206.33 & 7.92 & 20.67 & 48 & 3.44 & 0.001 & {$[8.58,32.76]$} & 0.96 & {$[0.37,1.54]$} \\
\hline IReST 4 & 25 & 244 & 32 & 214.31 & 11.18 & 29.69 & 48 & 4.38 & $<.001$ & {$[16.05,43.32]$} & 1.22 & {$[0.61,1.82]$} \\
\hline IReST 5 & 25 & 229 & 25 & 210.15 & 6.92 & 18.85 & 48 & 3.63 & 0.001 & {$[8.41,29.28]$} & 1.01 & {$[0.42,1.60]$} \\
\hline IReST 6 & 25 & 197 & 22 & 182.13 & 11.83 & 14.87 & 48 & 2.98 & 0.005 & {$[4.82,24.91]$} & 0.83 & {$[0.25,1.41]$} \\
\hline IReST 7 & 25 & 232 & 30 & 206.51 & 10.02 & 25.49 & 48 & 4.03 & $<.001$ & {$[12.77,38.21]$} & 1.12 & {$[0.52,1.72]$} \\
\hline IReST 8 & 25 & 237 & 24 & 215.82 & 9.67 & 21.18 & 48 & 4.09 & $<.001$ & {$[10.77,31.59]$} & 1.14 & {$[0.54,1.74]$} \\
\hline IReST 9 & 25 & 226 & 26 & 207.28 & 9.70 & 18.72 & 48 & 3.37 & 0.001 & {$[7.56,29.88]$} & 0.94 & {$[0.35,1.52]$} \\
\hline IReST 10 & 25 & 211 & 26 & 189.55 & 7.46 & 21.45 & 48 & 3.97 & $<.001$ & {$[10.57,32.33]$} & 1.10 & {$[0.50,1.70]$} \\
\hline
\end{tabular}


using a Holm-Bonferonni correction for multiple comparisons were used to compare the normative UK IReST data (means and standard deviations) to the current sample; the results showed statistically significant differences between the current samples mean reading speed on the IReST texts and the normative data provided by the UK IReST texts (see Table 3). In all cases $p$-values were equal to or less than 0.005. Mean difference scores ranged from $14.87 \mathrm{wpm}$ to $30.05 \mathrm{wpm}$, with $95 \%$ confidence intervals ranging from 4.82 to 43.32 (see Figure 4). Measures of effect size using bias corrected Hedge's $g^{*}$ ranged from 0.83 to 1.32, with 95\% confidence intervals ranging from 0.25 to 1.93 . Therefore, there are statistically significant differences in reading speed between the current sample and the UK sample, such that the NA English IReST values fall outside of the standards established by the UK English IReST.

The original UK IReST separated the 10 texts into 5 different performance categories, to determine which texts can be used to compare participants reading speeds based on clinically relevant differences of 10 wpm (see Table 4; Trauzettel-Klosinski \& Dietz, 2012). Applying these criteria to the NA IReST, generates only two performance categories, thereby increasing the number of comparable texts. More specifically, when using the NA IReST texts 1, 2, 3, 4, 5, 7,8 , and 9 can be used to compare individuals reading speeds, and texts 6 and 10 can be used to assess individuals reading speeds.

\section{Normal Vision Condition VS. Impaired Condition}

To test the hypothesis that individuals with simulated visual impairments would read the texts significantly slower then individuals with normal or corrected to normal vision, mean reading speeds in wpm were calculated for each text in the normal and impaired vision conditions (see Table 5). Multiple two-sample dependent t-tests using a Holm-Bonferonni correction for multiple comparisons were used to compare the mean reading speed in wpm of the normal and impaired vision conditions; the results showed statistically significant differences between the mean reading speeds of the normal vision condition and the simulated impairment condition on the IReST texts (see Table 5). In all cases, the $p$-values were less than 0.001. Mean difference scores ranged from $25.44 \mathrm{wpm}$ to $41.8 \mathrm{wpm}$, with $95 \%$ confidence intervals ranging from 21.66 to 46.18 (see Figure 5). Measures of effect size using bias corrected Hedge's $g^{*}$ ranged from 2.74 to 3.81 , with $95 \%$ confidence intervals ranging from 1.97 to 4.74 . Further Bayesian analyses revealed $\mathrm{BF}_{10}$ factors ranging from $1.277 \times 10^{7}$ to $7.334 \times 10^{11}$, indicating decisive evidence for the research hypothesis (see Table 6). Thus participants in the simulated 


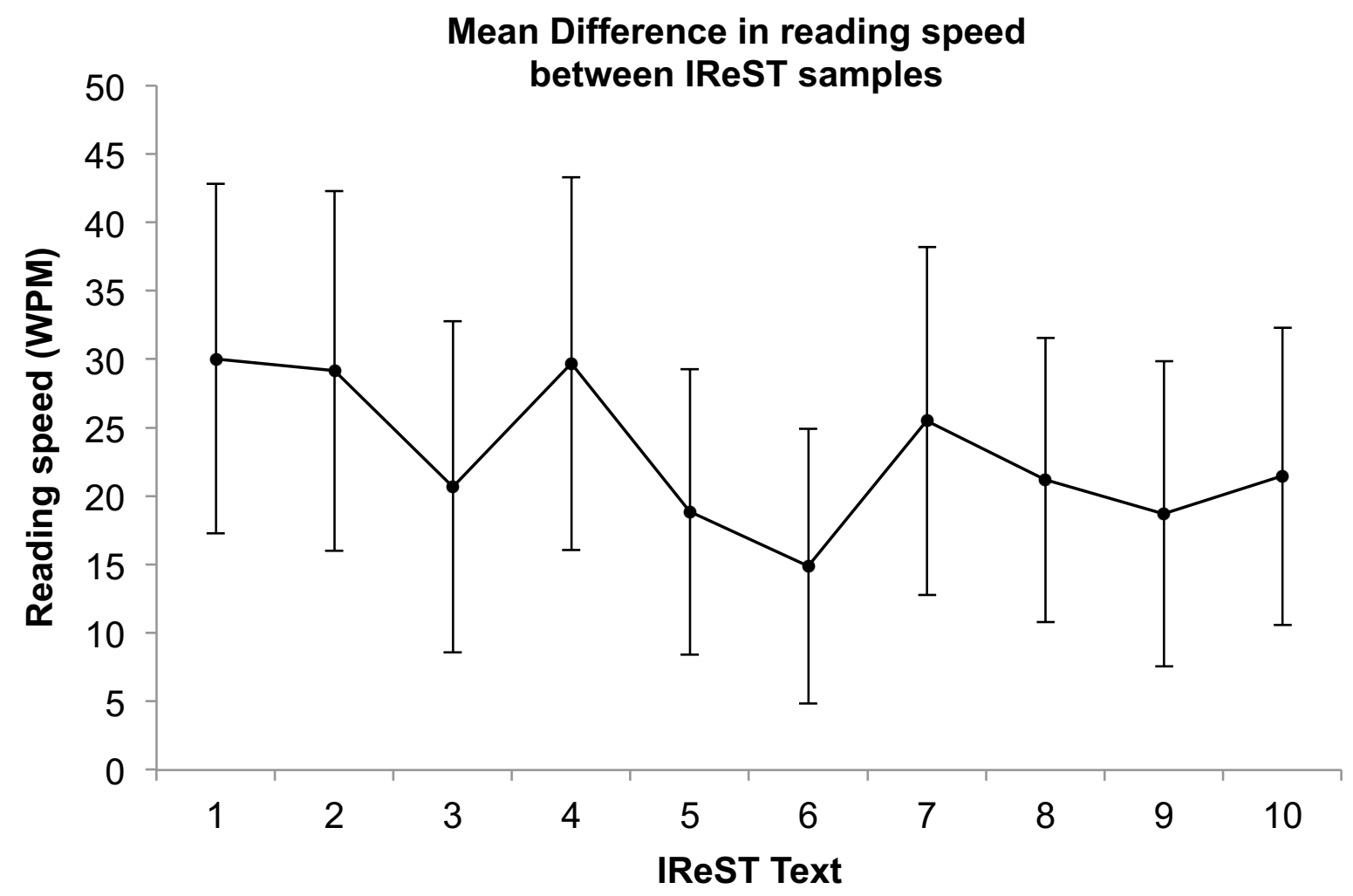

Figure 4. Mean difference in reading speeds in words per minute (WPM) between the NA and UK English samples. Error bars represent 95\% confidence intervals around the mean. 
Table 4.

Reading Speeds for Each Text, Ranked in Order of Decreasing Speed. Texts with the Same Letter in Performance Category Differ no More than 10 wpm

\begin{tabular}{|c|c|c|c|c|c|c|c|}
\hline Original IReST & $\begin{array}{l}\mathrm{IReST} \\
\text { text \# }\end{array}$ & $\begin{array}{l}\text { Rank } \\
\text { Order } \\
\end{array}$ & $\begin{array}{c}\text { Reading Speed } \\
(\mathrm{wpm})\end{array}$ & & \multicolumn{3}{|c|}{$\begin{array}{c}\text { Performance } \\
\text { Category }\end{array}$} \\
\hline & 4 & 1 & 244 & A & & & \\
\hline & 2 & 2 & 243 & $\mathrm{~A}$ & & & \\
\hline & 8 & 3 & 237 & A & $\mathrm{B}$ & & \\
\hline & 1 & 4 & 236 & A & $\mathrm{B}$ & & \\
\hline & 7 & 5 & 232 & & $\mathrm{~B}$ & $\mathrm{C}$ & \\
\hline & 5 & 6 & 229 & & $\mathrm{~B}$ & $\mathrm{C}$ & \\
\hline & 3 & 7 & 227 & & $\mathrm{~B}$ & $\mathrm{C}$ & \\
\hline & 9 & 8 & 226 & & & $\mathrm{C}$ & \\
\hline & 10 & 9 & 211 & & & & $\mathrm{D}$ \\
\hline & 6 & 10 & 197 & & & & \\
\hline
\end{tabular}

Normal Vision

$\begin{array}{ccccc}8 & 1 & 216 & \text { A } & \\ 4 & 2 & 214 & \text { A } & \\ 2 & 3 & 214 & \text { A } & \\ 5 & 4 & 210 & \text { A } & \\ 9 & 5 & 207 & \text { A } & \\ 7 & 6 & 207 & \text { A } & \\ 3 & 7 & 206 & \text { A } & \\ 1 & 8 & 206 & \text { A } & \\ 10 & 9 & 190 & & \text { B } \\ 6 & 10 & 182 & & \text { B }\end{array}$

Simulated Impairment

\begin{tabular}{ccccccc}
8 & 1 & 185 & A & & & \\
5 & 2 & 177 & A & B & & \\
3 & 3 & 176 & A & B & & \\
4 & 4 & 175 & & B & & \\
7 & 5 & 174 & & B & C & \\
2 & 6 & 172 & & B & C & \\
9 & 7 & 170 & & B & C & \\
1 & 8 & 168 & & B & C & \\
10 & 9 & 164 & & & C & \\
6 & 10 & 152 & & & & D \\
\hline
\end{tabular}


Table 5.

Results of the Two Sample Dependent t-test Between Reading Speeds on the IReST of the Normal and Impairment Conditions.

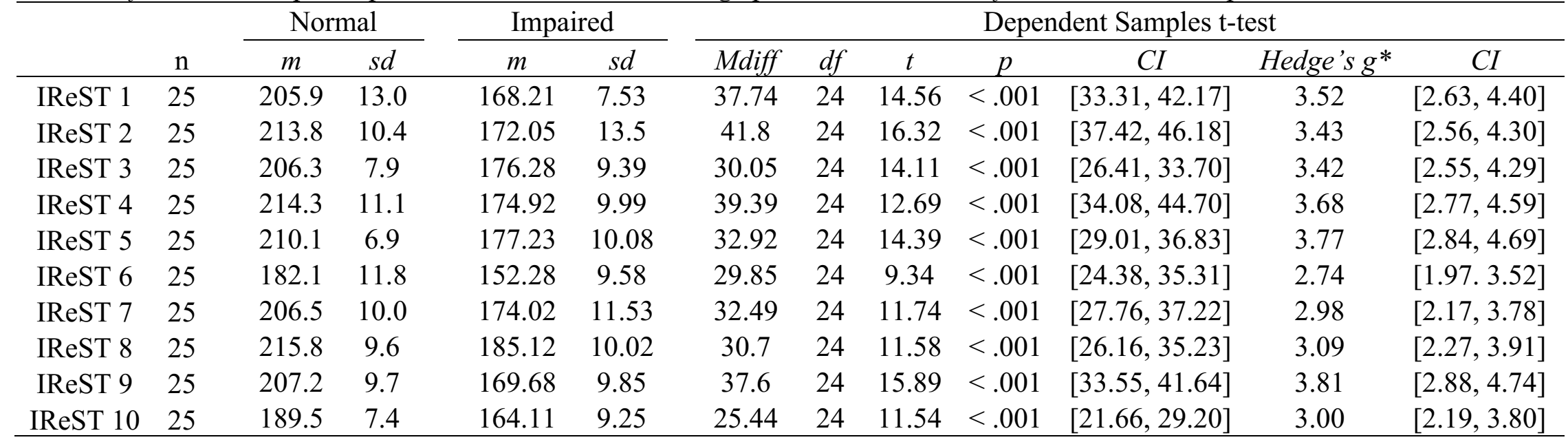




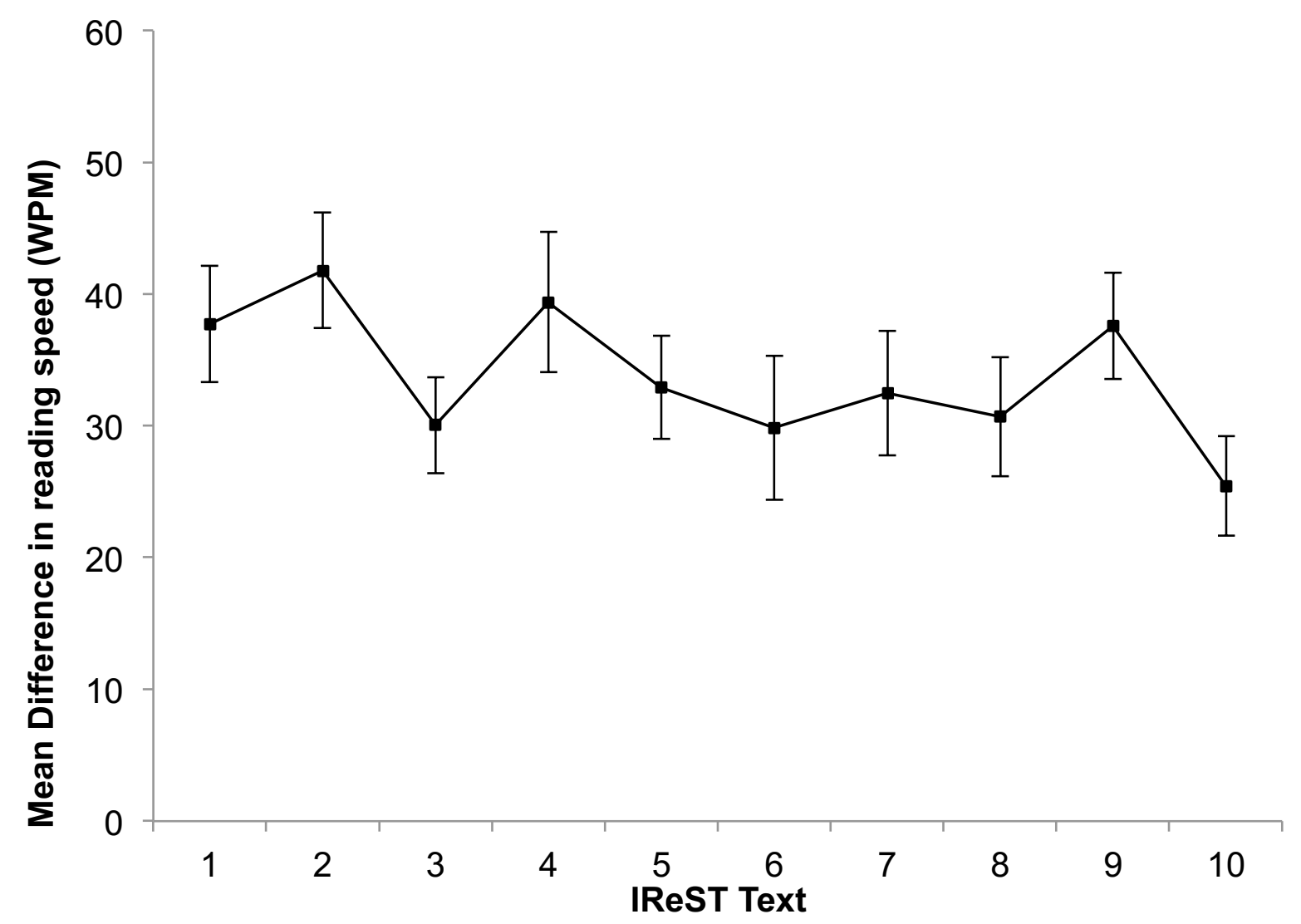

Figure 5. Mean difference in reading speeds in words per minute (WPM) between the normal and simulated impairment conditions. Error bars represent $95 \%$ confidence intervals around the mean. 
Table 6.

Results of Bayesian Analysis of Reading Speeds on the IReST of the Normal and Impairment Conditions.

\begin{tabular}{|c|c|c|c|c|}
\hline & \multicolumn{2}{|c|}{$\mathrm{BF}$} & \multicolumn{2}{|c|}{ Effect Size $(\delta)$} \\
\hline & $\mathrm{BF}_{10}$ & error $\%$ & Median & $\mathrm{CI}$ \\
\hline IReST 1 & $6.773 \times 10^{10}$ & $1.230 \times 10^{-17}$ & 2.79 & {$[1.90,3.74]$} \\
\hline IReST 2 & $7.334 \times 10^{11}$ & $2.627 \times 10^{-18}$ & 3.13 & {$[2.14,4.18]$} \\
\hline IReST 3 & $3.566 \times 10^{10}$ & $3.868 \times 10^{-17}$ & 2.74 & {$[1.85,3.63]$} \\
\hline IReST 4 & $4.187 \times 10^{9}$ & $5.097 \times 10^{-16}$ & 2.43 & {$[1.65,3.26]$} \\
\hline IReST 5 & $5.331 \times 10^{10}$ & $1.804 \times 10^{-17}$ & 2.75 & {$[1.89,3.70]$} \\
\hline IReST 6 & $1.277 \times 10^{7}$ & $4.979 \times 10^{-13}$ & 1.77 & {$[1.13,2.45]$} \\
\hline IReST 7 & $9.121 \times 10^{8}$ & $3.341 \times 10^{-15}$ & 2.24 & {$[1.49,3.04]$} \\
\hline IReST 8 & $6.973 \times 10^{8}$ & $3.505 \times 10^{-13}$ & 2.2 & {$[1.47,3.00]$} \\
\hline IReST 9 & $4.188 \times 10^{11}$ & $4.752 \times 10^{-18}$ & 3.04 & {$[2.114,4.06]$} \\
\hline IReST 10 & $6.533 \times 10^{8}$ & $3.725 \times 10^{-17}$ & 2.2 & {$[1.46,2.97]$} \\
\hline
\end{tabular}


impairment condition read the IReST significantly slower than the normal vision condition.

\section{Discussion}

The purpose of this study was to investigate whether the normative values of the IReST are valid in an English-speaking NA sample. The results showed that there were statistically significant differences between normative values of the IReST and the mean reading speeds in wpm on each of the texts in the NA sample. As the mean reading speeds fell outside of the standards established by the UK English language IReST texts, the normative values provided by the IReST do not appear to be valid in an English-speaking NA sample. The purpose of the study was also to investigate the impact of visual impairments on the IReST through the use of a simulated visual impairment. A visual impairment of $0.5 \log$ Mar (20/60 Snellen acuity) was simulated using low vision simulator goggles; the results showed that the impact of the simulated visual impairment decreased reading speeds across texts between $25.44 \mathrm{wpm}$ to $41.8 \mathrm{wpm}$ when compared to the normal vision condition (see Figure 6). Therefore, neither the established UK IReST values nor the newly developed NA IReST values are likely valid in the true population of individuals with visual impairments.

There are four potential explanations for the results observed in the normal vision condition; the first is that there are true regional differences in the way English is processed in the UK and North America, which would confirm the findings of Jacewicz et al. (2009) and Whiteside (1996). This would mean that the IReST would require normative values based on different regions for the same language. The second is that the differences in reading speed are attributable to the content of the text; i.e., as the texts are written in UK English and use terms and phrases that are unfamiliar to NA English speakers, it is these semantic differences that impact participants reading ability and automaticity that results in a decrease in reading speed. If this is the case, by adapting the IReST texts to use NA terms and phrases, then we would predict that the differences between the reading speeds of the UK sample and the NA sample should no longer be significant. The third explanation is that even though the sample consisted of participants whose first and dominant languages were English, the entire sample was bilingual, and the majority of the sample was trilingual (54\%). Therefore, according to the weaker links hypothesis (Cop et al., 2015; Gollan et al., 2008), this may account for the differences in reading speed. However, competing theories suggest differences in reading speed only occur in 


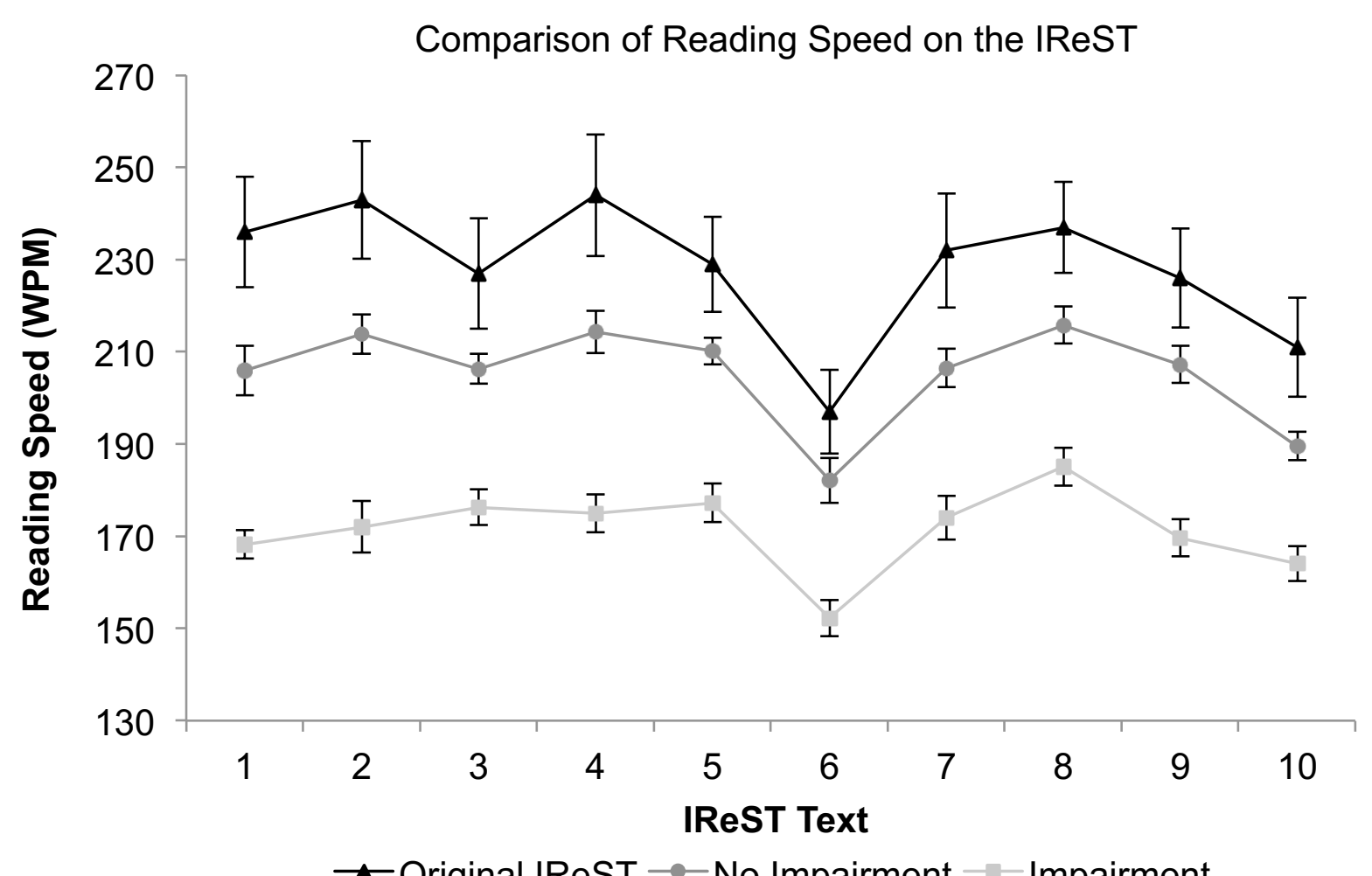

Figure 6. Mean reading speeds in words per minute (WPM) across IReST texts. Error bars represent $95 \%$ confidence intervals around the mean 
individuals second and/or third languages, and that this will only occur if they do not have sufficient language proficiency (Cop et al., 2015; Favreau \& Segalowitz, 1983; Segalowitz \& Hulstijn, 2005). Finally, the fourth explanation is that a combination of all of these factors are influencing participants reading speed. In this case, special considerations will need to be taken when choosing which normative values to use when comparing performance on the IReST, and in which language the assessment should be administered for optimal assessment (Lezak et al., 2012).

Of note is that in both the normal vision condition and the simulated impairment condition the overall pattern of the results remains constant; that is, across texts 1 through 10 the reading speeds of the NA sample are consistent with the reading speeds of the UK sample but shifted downwards on average by 23 and 34 wpm respectively (see Figure 6). However, unlike the original IReST values, in the NA sample, the majority of the texts are comparable, and this is likely due to the removal of between-subject variability. Removing the between-subject variability of the natural differences in individual reading speed between participants allowed us to only examine the variance attributable to differences in reading speed caused by the texts themselves; thereby providing a more accurate measure of which texts are comparable. By doing this, and by following the guidelines established by Trauzettel-Klosinski and Dietz (2012), we found that in the normal vision condition IReST texts $1,2,3,4,5,7,8$, and 9 are directly comparable to one another, and that texts 6 and 10 are directly comparable to one another. In the simulated impairment condition, there was greater variability in the mean reading speeds of the texts, and therefore there are four performance categories of comparable texts (see Table 4). In the impaired condition reading speeds were significantly slower compared to the normal vision condition. Thus when assessing individuals with sensory impairments, neither the UK nor the NA normative IReST values should be used as a basis of comparison. It is important to note, however, that the values that have now been established in the impairment condition may also not be accurate measures of low vision reading speed, as participants were given simulated visual impairments. As previously mentioned, individuals with sensory impairments may actually have comparable reading speeds to the normal vision condition as they have had time to get used to their visual impairment and may have come up with compensatory strategies to increase their reading speed (Crossland et al., 2004; Nguyen et al., 2011). To determine if there were true differences between a simulated impairment condition and a true sample of 
individuals with sensory impairment, the original IReST values, the normal vision condition, and the impaired vision condition, were compared to available data from a previous study assessing the Apple iPad as a tool for low vision (see Figure 7; Morrice, Johnson, Marinier, \& Wittich, 2017). Here reading speed in wpm is compared across conditions for texts $1,2,4$, and 8 , as those were the only texts used in the iPad study. There are significant differences between the simulated impairment conditions and the actual impairment group, such that the actual impairment condition had significantly slower reading speeds. This finding would suggest that the simulated impairment condition in the current study is actually an under-estimate of individuals with sensory impairments reading speed, and that future studies should focus on developing normative values for this population. This underestimate may be in part due to the iPad study consisting mostly of older adults (50+), and more specifically, older adults with sensory impairments.

One of the limitations of the present study is that the IReST texts were validated on a sample of undergraduate students between the ages of 18 and 45 years old, therefore there are still no normative values for older adults $(50+)$. However, as the purpose of the study was to validate the normative values established by Trauzettel-Klosinski and Dietz (2012), the sample in this study is comparable to the one that was used to establish the original normative values, i.e., undergraduates between the ages of 18 and 35. Therefore, future studies should examine whether older adults $(50+)$ in North America with normal, or corrected to normal vision, reading speed on the IReST fall outside of the normative values established in this study. It would be hypothesized that older adults with and without visual impairment would have significantly slower reading speeds on the IReST texts compared to younger adults with normal, or corrected to normal vision. Another limitation is the use of a simulated visual impairment; as it appears that the values established in the impairment condition under-estimate the reading speed of individuals with sensory impairments then these values may not reflect the reading speed of individuals with true visual impairments. Future studies should examine the impact of true visual impairments on reading speed on the IReST texts. Additionally, future studies should examine the validity of the low vision simulator goggles used in this study, as there are to my knowledge, no studies that have examined their efficacy and none that have used them in the assessment of reading speed.

The aim of the current study was to investigate whether the normative values of the 


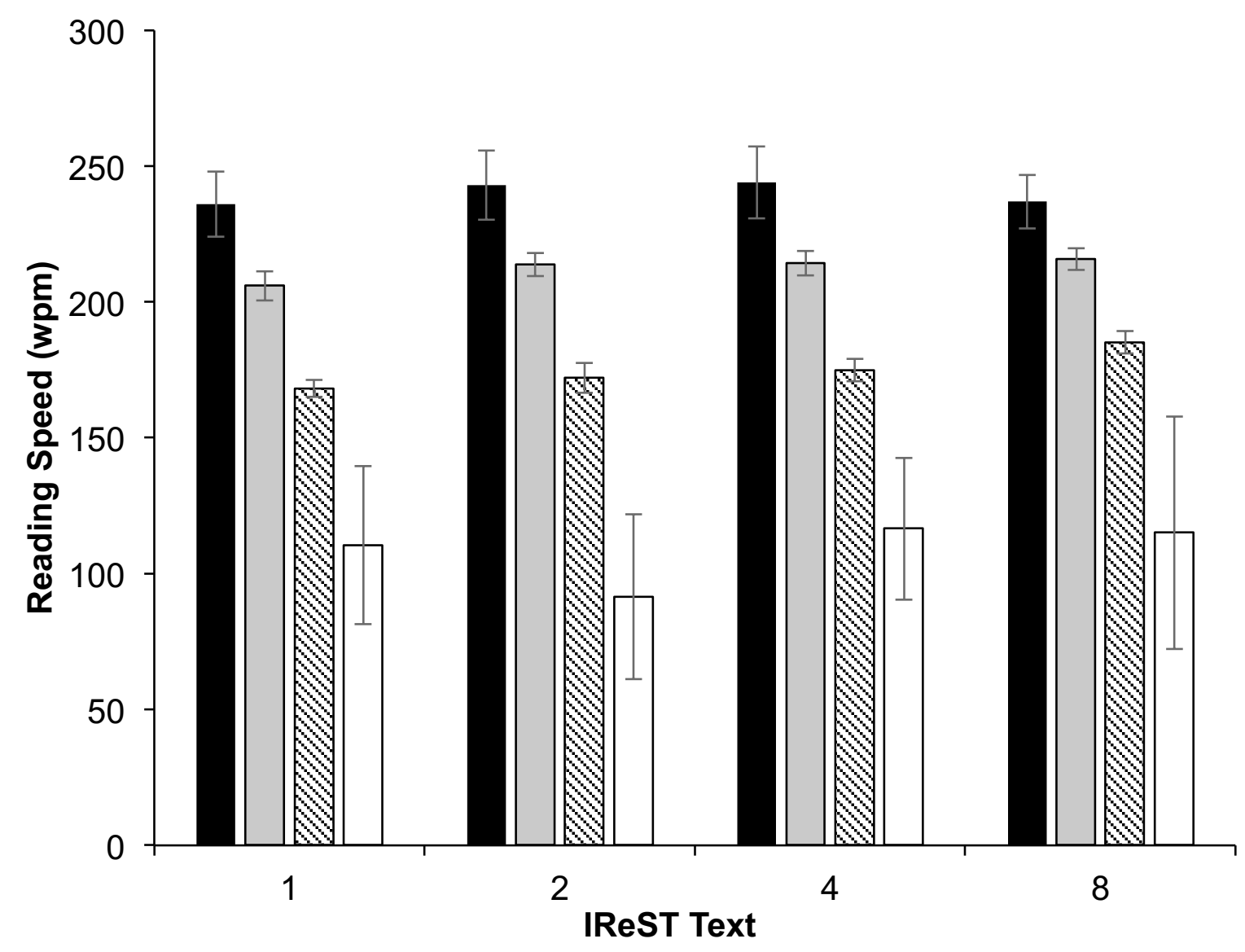

- Original $\square$ Normal Vision $\quad$ Simulated Impairment $\square$ Impairment

Figure 7. Mean reading speeds in words per minute (WPM) on IReST texts 1, 2, 4, and 8 across all conditions and compared to the reading speeds of older adults with real sensory impairments. Error bars represent $95 \%$ confidence intervals around the mean. 
IReST texts are valid in an English-speaking NA sample, as well as to examine the impact of a simulated sensory impairment on reading speed on the IReST. When interpreting the findings, it appears that the reading speeds of NA English speakers do fall outside of the normative values established by UK English IReST texts, and that simulated visual impairments do significantly decrease reading speed of individuals when reading the IReST. Therefore, when assessing reading speed using the IReST in a NA sample, we would recommend that researchers and clinicians use the values established in this study for a normative point of comparison, and not the original IReST values that are printed on the IReST cards. Finally, when assessing individuals with sensory impairments it is recommended that researchers and clinicians use the values established in the simulated visual impairment condition as a guide, and be wary that the true reading speed of individuals with sensory impairments are likely significantly lower than the values established here. 


\section{Experiment 2}

The purpose of Experiment 1 was to validate the IReST in a NA sample. However, as noted in the general introduction, there is a high proportion bilingual speakers in NA, e.g., English/French or English/Spanish bilinguals. Consequently, Experiment 2 will examine the impact of language fluency on the IReST to investigate whether the normative values identified in the NA English IReST are robust enough to reliably account for differences in language background and fluency when assessing a multilingual sample of English speaking North Americans, i.e., L1, L2, and L3 English speakers.

Assessing the impact of language fluency on the IReST is important as there are a high proportion of L2 and L3 English speakers in North America, for example within Quebec, 44.6\% of the population reports that they are English/French bilinguals (Statistics Canada, 2017). As such, in Quebec low vision rehabilitation services generally assess clients in either English or French (Overbury \& Wittich, 2011; Robillard \& Overbury, 2006). However, a secondary analysis of demographic data from Overbury \& Wittich (2011) reveals that of the individuals who access low vision rehabilitations services in Montreal, 28\% had first languages that were neither English nor French. Thus, these clients were assessed in their non-dominant language, which may have impacted their performance on the assessments used in low vision rehabilitation settings, as well as the outcome of their rehabilitation. Consequently, an important consideration when assessing individuals on standardized measures used in low vision rehabilitation, e.g., the IReST, is whether administering the assessment to individuals in their non-dominant language represents a minor or extensive deviation from standardization protocols (Lezak et al., 2012; Sattler, 1969, 2008). Minor deviations in test procedures, e.g., time of day of the assessment, may be less likely to impact the results of an assessment compared to extensive deviations from protocols, e.g., administering an assessment to a client in their non-dominant language (Sattler, 2008).

A number of researchers have investigated the impact of assessing individuals in their non-dominant language on standardized tests; for example, a study by Gasquoine, Croyle, Cavazos-Gonzalez, and Sandoval (2007) examined this by assessing the performance of Spanish/English bilinguals on both Spanish and English versions of neuropsychological assessments. Participants were separated into groups of either Spanish-dominant bilinguals, balanced bilinguals, or English-dominant bilinguals. The results of the study showed that there 
were no significant differences between balanced bilinguals scores on both the Spanish and English versions of the assessments. However, there were significant differences between scores on assessments for Spanish-dominant and English-dominant bilinguals, such that they scored lower on assessments that have a greater focus on language, e.g., the Woodcock Munoz Language Survey-Revised (Alvarado, Ruef, \& Schrank, 2005). Therefore, it would appear that assessing an individual in their non-dominant language may represent a minor deviation from standardization protocols if they have a high level of fluency in that language. Conversely, assessing an individual with low fluency in their non-dominant language may represent an extensive deviation from standardization protocols, as this would significantly impact their performance.

Thus the aim of Experiment 2 was to examine the impact of language fluency on the IReST and determine: (1) whether the reading speeds of a sample of multilingual English speakers falls outside of the normative values of the NA English IReST; and (2) the impact of a simulated sensory impairment on reading speed in this sample. In this experiment, normally sighted multilingual English speakers will be asked to read all 10 English language IReST texts following the same protocol used in Experiment 1. It was hypothesized that; (1) the mean reading speeds of a sample of multilingual English speakers will fall below than the normative values of the NA English IReST; and (2) that the simulated visual impairments would further reduce mean reading speeds across texts.

\section{Method}

The research protocol was approved by the research ethics board of Concordia University (certificate 30003975), in accordance with the Canadian Tri-Council Policy Statement of ethical conduct for research involving humans. Experiment 2 used the same method as experiment 1 , therefore, the same measures, materials, and procedures were used.

\section{Participants}

Fifty undergraduate students $(n=50)$ between the ages of 19 and $37(M=22.40, S D=3.30)$ were recruited from Concordia University to participate in this study. Participants were recruited using the Concordia University Psychology Participant Pool and were granted course credit for participating in this study. Participants were required to have normal, or corrected-to-normal, vision. See Table 7 for participant demographics. 
Table 7.

Participant demographics

\begin{tabular}{|c|c|c|}
\hline Demographics & $n$ & $\%$ \\
\hline Total Survey Respondents $(n=50)$ & & $100 \%$ \\
\hline Male & 3 & $6 \%$ \\
\hline Female & 47 & $94 \%$ \\
\hline \multicolumn{3}{|l|}{ Age } \\
\hline $18-25$ & 45 & $90 \%$ \\
\hline $26-35$ & 4 & $8 \%$ \\
\hline $36-45$ & 1 & $2 \%$ \\
\hline \multicolumn{3}{|l|}{ First Language } \\
\hline English & 15 & $30 \%$ \\
\hline French & 20 & $40 \%$ \\
\hline Other & 15 & $30 \%$ \\
\hline \multicolumn{3}{|l|}{$\underline{\text { Second Language }}$} \\
\hline English & 28 & $56 \%$ \\
\hline French & 20 & $40 \%$ \\
\hline Other & 2 & $4 \%$ \\
\hline \multicolumn{3}{|l|}{ Third Language $(n=35)$} \\
\hline English & 3 & $8.6 \%$ \\
\hline French & 6 & $17.1 \%$ \\
\hline Other & 26 & $74.3 \%$ \\
\hline \multicolumn{3}{|c|}{ Visual impairment not corrected through lenses } \\
\hline Yes & 0 & $0 \%$ \\
\hline No & 50 & $100 \%$ \\
\hline \multicolumn{3}{|l|}{ Known hearing impairment } \\
\hline Yes & 0 & $0 \%$ \\
\hline No & 50 & $100 \%$ \\
\hline \multicolumn{3}{|l|}{ Reading/Attention Disability } \\
\hline Yes & 1 & $2 \%$ \\
\hline No & 49 & $98 \%$ \\
\hline
\end{tabular}




\section{Results}

\section{Data Analysis}

As in experiment 1, the data in experiment 2 were combined to create 25 metaparticipants for the normal and impaired vision conditions and participants between-subject variability was removed. A Bayesian two-sample dependent (or paired) t-test was used to determine: (1) whether the low vision simulator goggles impaired participant's visual acuity and contrast sensitivity to the same degree throughout the experiment; (2) whether the reading speeds of a sample of multilingual English speakers fell below the normative values of the NA IReST; and (3) whether the reading speeds of a sample of multilingual English speakers wearing lowvision simulator goggles fell below the normative values of the simulated impairment condition of the NA IReST. Bayes factors (BF) were calculated using JASP 0.8.0.1 (JASP Team, 2016), and for each analysis a Cauchy prior width of 0.707 was used. For each analysis, the robustness of the posterior was checked using wider priors of 1.00 and 1.50 to ensure that the choice of prior distribution did not significantly impact the results. As in experiment 1 the results here are reported as both traditional NHST, and using Bayesian two-sample dependent t-tests, along with measures of effect sizes and confidence intervals so that the reader may interpret their statistic of choice.

\section{Descriptive Statistics}

The majority of participants (70\%) first language was not English, however, $56 \%$ of participants reported that their dominant language was English. One hundred percent of L1 English participants reported they were fluent English readers $(m=5, s d=0), 75 \%$ of L1 French participants reported they were fluent English readers $(m=4.75, s d=.44)$, and $60 \%$ of L1 Other participants reported they were fluent English readers $(m=4.6, s d=.51)$. All participants $(100 \%)$ were bilingual, and $70 \%$ of participants were trilingual. See Table 8 for a breakdown of participants self-reported language fluency (speaking, reading, writing, and listening ability) in English, French, and third languages.

In the normal vision condition, $78 \%$ of the reading comprehension questions were answered correctly and in the simulated impairment condition $82 \%$ of the questions were answered correctly. See Appendix C for a breakdown of the proportion of questions answered correctly for each of the texts. Similar to what occurred in experiment 1, it appeared that participants experienced difficulty answering the reading comprehension question for text 2 , and 
Table 8.

Language Fluency

\begin{tabular}{|c|c|c|c|c|c|c|c|}
\hline \multirow{6}{*}{$\begin{array}{l}\text { L1 } \\
\text { English } \\
(n=15)\end{array}$} & Language Ability & $n$ & No Ability & Elementary & Moderate & Very Good & Fluent \\
\hline & English & 15 & & & & & \\
\hline & Speaking & & 0 & 0 & 0 & 0 & 15 \\
\hline & Reading & & 0 & 0 & 0 & 0 & 15 \\
\hline & Writing & & 0 & 0 & 0 & 1 & 14 \\
\hline & Listening & & 0 & 0 & 0 & 0 & 15 \\
\hline
\end{tabular}

French

15

Speaking

Reading

Writing

Listening

Speaking

Reading

Writing

French

Listening

0
0
1
0

4
7
6
5

3
1
3
0

$\begin{array}{ll}4 & 4 \\ 5 & 2 \\ 3 & 2 \\ 4 & 6\end{array}$

$(n=20)$

English

Speaking

Reading

Writing

Listening

9

French

20

Speaking

Reading

Writing

Listening

Other

Speaking

Reading

Writing

Listening

20

$\begin{array}{ll}0 & 5 \\ 3 & 2 \\ 3 & 1 \\ 0 & 3\end{array}$

3
3
5
4

0

0

0

$\begin{array}{ll}0 & 0 \\ 2 & 0\end{array}$

$\begin{array}{lllll}0 & 3 & 0 & 7 & 10 \\ 0 & 0 & 0 & 5 & 15 \\ 0 & 0 & 0 & 7 & 13 \\ 0 & 0 & 0 & 3 & 17\end{array}$

10

13

17

$\begin{array}{lllll}0 & 1 & 0 & 5 & 14 \\ 0 & 2 & 0 & 3 & 15 \\ 0 & 3 & 0 & 5 & 12 \\ 0 & 0 & 0 & 0 & 20\end{array}$

10 
Table 8. cont.

Language Fluency

\begin{tabular}{llllllll}
\hline L1 & Language Ability & $n$ & No Ability & Elementary & Moderate & Very Good & Fluent \\
\hline
\end{tabular}

Other

$(n=15)$

English

15

Speaking

Reading

Writing

Listening

French

Speaking

Reading

Writing

Listening

0
0
0
0

0

6

9

$0 \quad 0$

6

9

0

$4 \quad 11$

0

3

12

15

Other

14

Speaking

Reading

Writing

Listening

$\begin{array}{ll}0 & 6 \\ 0 & 3 \\ 0 & 6 \\ 1 & 4\end{array}$

$\begin{array}{ll}6 & 1\end{array}$

5

3

30

62

6

2

6

40

4

5

6

Note: Of the 33 participants with third languages, these languages included: Arabic, Creole,

English, French, German, Greek, Italian, Persian, Portuguese, Spanish, Telugu, and Urdu. 
again it is believed that this difficulty reflects the poor quality of the question.

\section{Visual Acuity and Low Vision Simulator Goggles}

Participants' visual acuity and contrast sensitivity were measured at three time points: (1)at baseline without the simulator goggles in the normal vision condition, (2) before reading the texts with the simulator goggles in the impaired condition, and (3) after reading the texts with the simulator goggles in the impaired condition. At the first time-point, participants had normal, or corrected to normal, vision, with an average visual acuity of $-0.11 \log \operatorname{MAR}(M=-0.11$, $S D=0.07, C I[-0.13,-0.09])$ and an average contrast sensitivity of $2.01 \log C S(M=2.01, S D=0.16$, $C I[1.96,2.06])$, see Figure 8. Again, the low vision simulator goggles failed at both the second and third time-points at simulating a 20/80 visual impairment, however, they were successful at inducing a simulated moderate visual impairment of 20/70, or $0.545 \log$ MAR. At the second time-point the goggles simulated a visual acuity of $0.54 \log \mathrm{MAR}(M=0.54, S D=0.16, C I[0.49$, $0.59])$ and contrast sensitivity of $.89 \log C S(M=.89, S D=.23, C I[0.83,0.96])$, and at the third time-point the goggles simulated a visual acuity of $0.55 \log \operatorname{Mar}(M=0.55, S D=0.14, C I[0.51$, $0.59])$ and contrast sensitivity of $0.90 \log C S(M=0.90, S D=0.17, C I[0.85,0.95])$. There were no significant differences between the participants visual acuity at the second and third time-points, $t(49)=-0.63, p>.05, d=-0.09, C I[-0.08,0.04]$; further Bayesian analysis revealed a $\mathrm{BF}_{01}$ factor of 5.38, indicating that the probability of the null hypothesis is 5.38 times greater than the probability of the research hypothesis. There were also no significant differences between the participants contrast sensitivity at the second and third time points, $t(49)=-0.14, p>.05, d=$ $0.02, C I[-0.08,0.07]$; further, Bayesian analysis revealed a $\mathrm{BF}_{01}$ factor of 6.44 , indicating that the probability of the null hypothesis is 6.44 times greater than the probability of the research hypothesis. Therefore, participants received the same degree of simulated visual impairment across the impairment condition.

\section{Multilingual Sample Compared to the Normative NA IReST Values}

To test the hypothesis that a multilingual English-speaking sample would read the IReST texts significantly slower then L1 English speaking sample from the NA IReST, mean reading speeds in wpm were calculated for each text (see Table 9). Multiple two-sample dependent ttests using a Holm-Bonferonni corrections for multiple comparisons were used to compare the mean reading speed in wpm of the multilingual and L1 samples; the results showed that there were no statistically significant differences between the mean reading speeds of the multilingual 

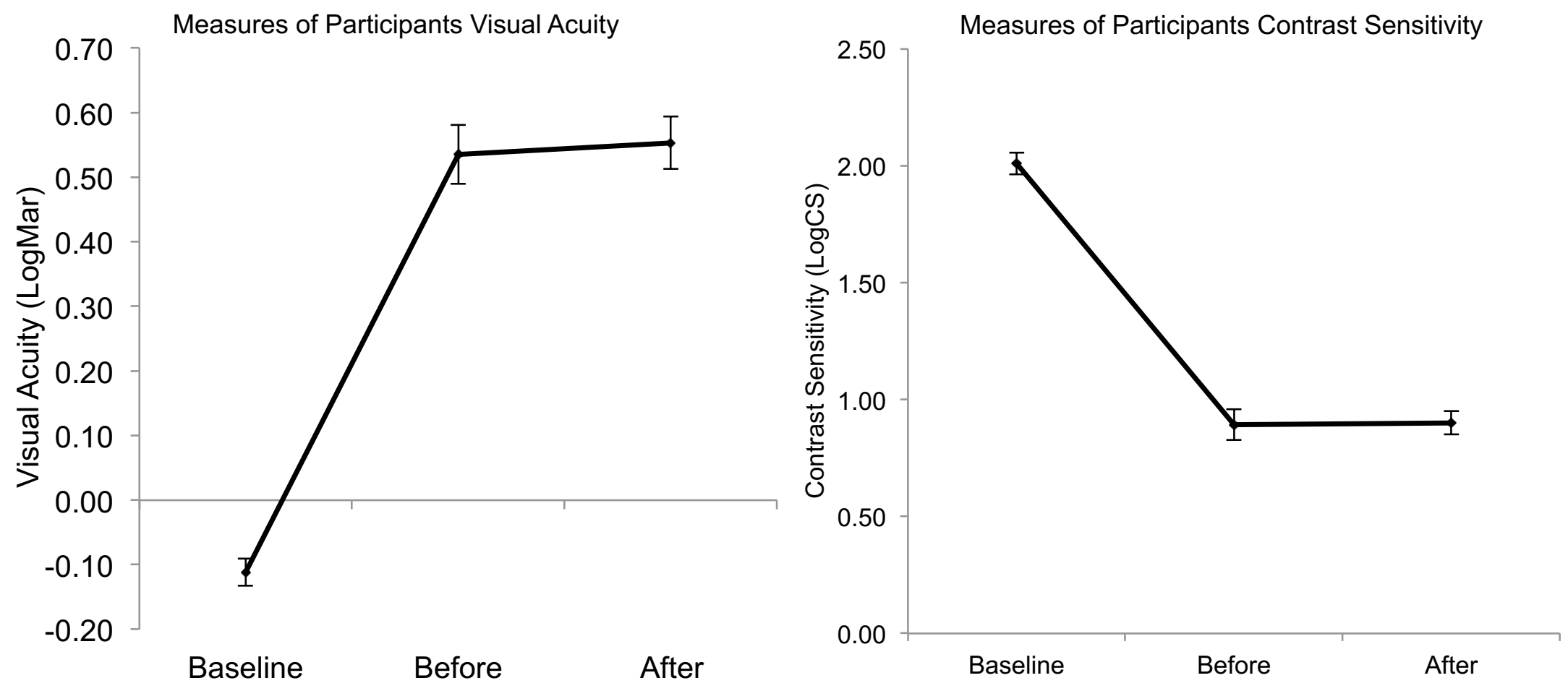

Figure 8. Measures of participant's visual acuity and contrast sensitivity. These figures represent changes in participant's visual acuity and contrast sensitivity from baseline to before and after the simulated visual impairment conditions. Error bars represent $95 \%$ confidence intervals around the mean. 
Table 9.

Results of the Two Sample Dependent t-test Between Reading Speeds on the IReST of the Multilingual English Sample and the Normative Values of the NA English IReST.

\begin{tabular}{|c|c|c|c|c|c|c|c|c|c|c|c|c|}
\hline & \multirow[b]{2}{*}{$\mathrm{n}$} & \multicolumn{2}{|c|}{$\begin{array}{l}\text { NA IReST - } \\
\text { Normal }\end{array}$} & \multicolumn{2}{|c|}{$\begin{array}{l}\text { Multilingual - } \\
\text { Normal }\end{array}$} & \multicolumn{7}{|c|}{ Dependent Samples t-test } \\
\hline & & $m$ & $s d$ & $m$ & $s d$ & Mdiff & $d f$ & $t$ & $p$ & $C I$ & Hedge's g* & $C I$ \\
\hline IReST 1 & 25 & 205.9 & 13.0 & 203.17 & 13.8 & 2.79 & 24 & 0.73 & $>.05$ & {$[-3.75,9.33]$} & 0.21 & {$[-0.35,0.76]$} \\
\hline IReST 2 & 25 & 213.8 & 10.4 & 210.69 & 12.6 & 3.16 & 24 & 1.11 & $>.05$ & {$[-1.71,8.03]$} & 0.27 & {$[-0.29,0.83]$} \\
\hline IReST 3 & 25 & 206.3 & 7.9 & 203.15 & 7.8 & 3.17 & 24 & 1.58 & $>.05$ & {$[-0.27,6.62]$} & 0.40 & {$[-0.16,0.96]$} \\
\hline IReST 4 & 25 & 214.3 & 11.1 & 213.13 & 8.7 & 1.18 & 24 & 0.42 & $>.05$ & {$[-3.62,5.98]$} & 0.12 & {$[-0.44,0.67]$} \\
\hline IReST 5 & 25 & 210.1 & 6.9 & 203.78 & 10.3 & 6.37 & 24 & 2.66 & .007 & {$[2.27,10.47]$} & 0.72 & {$[0.15,1.29]$} \\
\hline IReST 6 & 25 & 182.1 & 11.8 & 180.61 & 11.0 & 1.52 & 24 & 0.48 & $>.05$ & {$[-3.88,6.92]$} & 0.13 & {$[-0.42,0.69]$} \\
\hline IReST 7 & 25 & 206.5 & 10.0 & 201.54 & 9.1 & 4.97 & 24 & 2.75 & .006 & {$[1.87,8.06]$} & 0.51 & {$[-0.05,1.08]$} \\
\hline IReST 8 & 25 & 215.8 & 9.6 & 211.43 & 9.7 & 4.39 & 24 & 1.65 & $>.05$ & {$[-0.15,8.94]$} & 0.45 & {$[-0.11,1.01]$} \\
\hline IReST 9 & 25 & 207.2 & 9.7 & 202.37 & 14.4 & 4.91 & 24 & 1.57 & $>.05$ & {$[-0.43,10.25]$} & 0.40 & {$[-0.16,0.96]$} \\
\hline IReST 10 & 25 & 189.5 & 7.4 & 183.44 & 14.3 & 6.11 & 24 & 1.93 & .03 & {$[0.68,11.53]$} & 0.53 & {$[-0.03,1.09]$} \\
\hline
\end{tabular}


and L1 samples on IReST texts 1, 2, 3, 4, 6, 8, and 9 (see Table 9). Mean difference scores ranged from $1.18 \mathrm{wpm}$ to $4.91 \mathrm{wpm}$, with $95 \%$ confidence intervals ranging from -3.88 to 10.25 (see Figure 9). Measures of effect size using bias corrected Hedge's $g$ * ranged from 0.12 to 0.45 , with $95 \%$ confidence intervals ranging from -0.44 to 1.01 . Further Bayesian analyses revealed $\mathrm{BF}_{10}$ factors ranging from 0.30 to 1.30 , indicating anecdotal evidence for the null hypothesis in texts 1 and 2, substantial evidence for the null in texts 4 and 6, and anecdotal evidence for the research hypothesis in texts 3,8 and 9 (see Table 10). There were statistically significant differences between the mean reading speeds of the multilingual and L1 samples on IReST texts 5, 7, and 10 (see Table 9). In these texts, p-values were less than .03, and mean difference scores ranged from 4.97-6.37 with 95\% confidence intervals ranging from 0.68 to 11.53 (see Figure 9). Measures of effect size using bias corrected Hedge's $g^{*}$ ranged from 0.51 to 0.72 , with $95 \%$ confidence intervals ranging from -0.05 to 1.29 . Further Bayesian analyses revealed $\mathrm{BF}_{10}$ factors ranging from 1.98 to 8.61, indicating anecdotal evidence for the research hypothesis in text 10, and substantial evidence for the research hypothesis in texts 5 and 7 (see Table 10). Therefore, there were no statistically significant differences between the reading speeds of the NA IReST established in experiment 1 and reading speeds on IReST texts, 1, 2, 3, 4, 6, 8, and 9. However, reading speeds on IReST texts, 5, 6 and 10 were significantly lower than the values established in the NA IReST. Although there were no significant differences betweenthe majority of participants reading speeds on the IReST in this sample and the NA IReST, it is important to note that in most cases there was only anecdotal evidence for either the null or research hypothesis according to the Bayesian analysis.

\section{Impaired Multilingual Sample Compared to the Impaired Normative NA IReST Values}

To test the hypothesis that the multilingual English-speaking sample would read the IReST texts significantly slower than the L1 English speaking sample in the impaired condition, mean reading speeds in wpm were calculated for each text (see Table 11). Multiple two-sample dependent t-tests using a Holm-Bonferonni corrections for multiple comparisons were used to compare the mean reading speed in wpm of the multilingual and L1 samples; the results showed there were no statistically significant differences between the mean reading speeds of the multilingual and the L1 English speaking samples in the simulated impairment condition on the IReST texts (see Table 11). In all cases p-values were greater than 0.05. Mean difference scores ranged from $-6.62 \mathrm{wpm}$ to $2.42 \mathrm{wpm}$, with $95 \%$ confidence intervals ranging from -12.67 to 9.08 


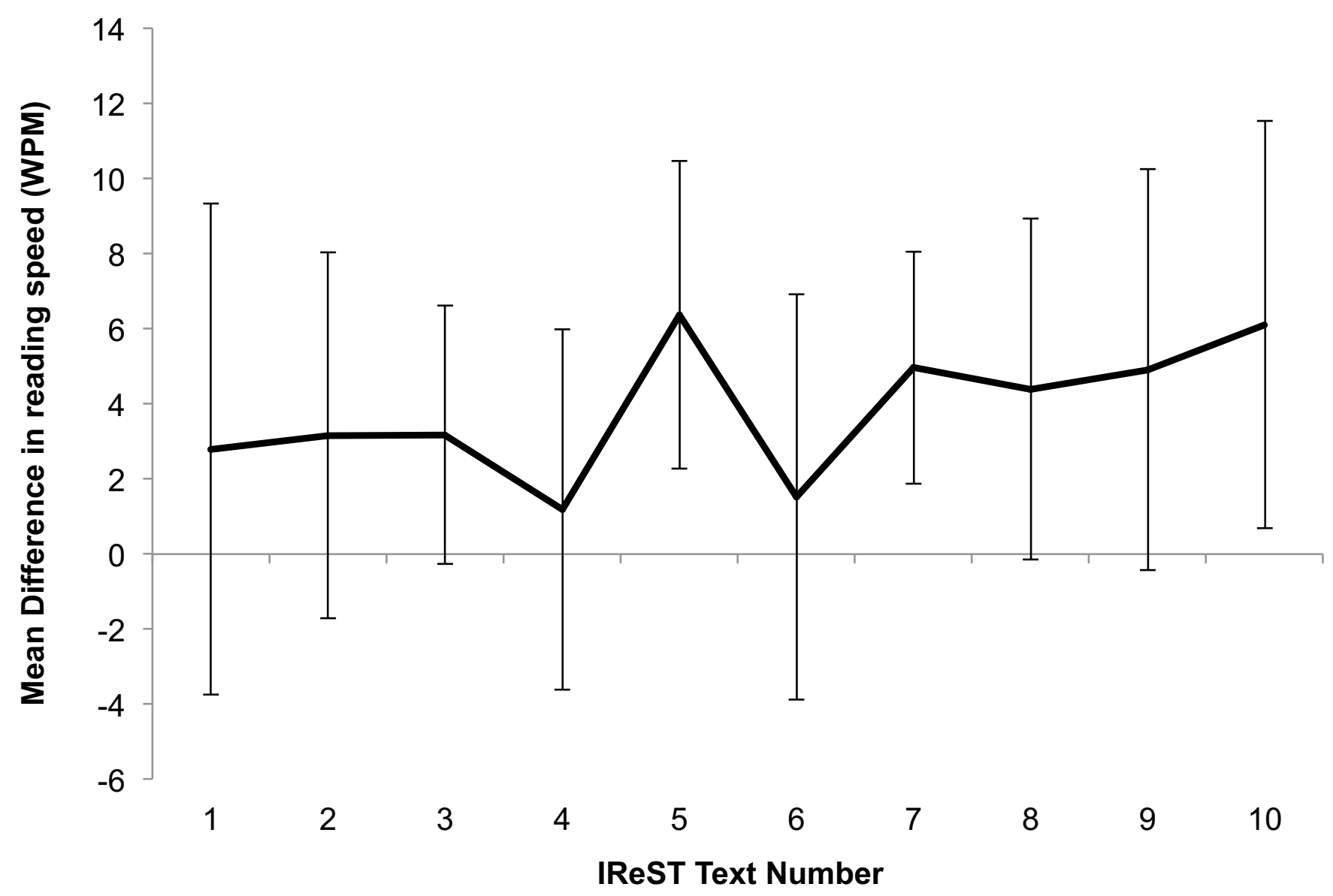

Figure 9. Mean difference in reading speeds in words per minute (WPM) between the multilingual English sample and the normative values of the NA English IReST. Error bars represent 95\% confidence intervals around the mean. 
Table 10.

Results of the Bayesian Analysis of Reading Speeds on the IReST of the Multilingual English Sample and the Normative Values of the NA English IReST.

\begin{tabular}{|c|c|c|c|c|}
\hline & \multicolumn{2}{|c|}{$\mathrm{BF}$} & \multicolumn{2}{|c|}{ Effect Size $(\delta)$} \\
\hline & $\mathrm{BF}_{10}$ & error $\%$ & Median & $\mathrm{CI}$ \\
\hline IReST 1 & 0.404 & $3.418 \times 10^{-4}$ & 0.18 & {$[0.01,0.53]$} \\
\hline IReST 2 & 0.622 & $7.207 \times 10^{-7}$ & 0.23 & {$[0.02,0.59]$} \\
\hline IReST 3 & 1.162 & $6.517 \times 10^{-7}$ & 0.30 & {$[0.01,0.67]$} \\
\hline IReST 4 & 0.300 & $3.085 \times 10^{-4}$ & 0.16 & {$[0.01,0.49]$} \\
\hline IReST 5 & 7.278 & $1.486 \times 10^{-5}$ & 0.48 & {$[0.11,0.90]$} \\
\hline IReST 6 & 0.317 & $3.145 \times 10^{-4}$ & 0.18 & {$[0.01,0.50]$} \\
\hline IReST 7 & 8.606 & $8.870 \times 10^{-6}$ & 0.50 & {$[0.12,0.90]$} \\
\hline IReST 8 & 1.300 & $6.389 \times 10^{-7}$ & 0.31 & {$[0.03,0.69]$} \\
\hline IReST 9 & 1.153 & $6.527 \times 10^{-7}$ & 0.30 & {$[0.03,0.68]$} \\
\hline IReST 10 & 1.982 & $5.915 \times 10^{-7}$ & 0.35 & {$[0.01,0.76]$} \\
\hline
\end{tabular}


Table 11.

Results of the Two Sample Dependent t-test Between Reading Speeds on the IReST of the Simulated Impairment Conditions of the Multilingual English Sample and the Normative Values of the NA English IReST.

\begin{tabular}{|c|c|c|c|c|c|c|c|c|c|c|c|c|}
\hline & \multirow[b]{2}{*}{$\mathrm{n}$} & \multicolumn{2}{|c|}{$\begin{array}{l}\text { NA IReST - } \\
\text { Impaired }\end{array}$} & \multicolumn{2}{|c|}{$\begin{array}{l}\text { Multilingual - } \\
\text { Impaired }\end{array}$} & \multicolumn{7}{|c|}{ Dependent Samples t-test } \\
\hline & & $m$ & $s d$ & $m$ & $s d$ & Mdiff & $d f$ & $t$ & $p$ & $C I$ & $\begin{array}{c}\text { Hedge's } \\
g^{*}\end{array}$ & $C I$ \\
\hline IReST 1 & 25 & 168.2 & 7.53 & 172.99 & 11.9 & -4.78 & 24 & -1.88 & $>.05$ & {$[-9.13,-0.44]$} & 0.47 & {$[-0.08,1.04]$} \\
\hline IReST 2 & 25 & 172.1 & 13.5 & 172.40 & 9.1 & -0.34 & 24 & -0.13 & $>.05$ & {$[-4.83,4.15]$} & 0.03 & {$[-0.52,0.58]$} \\
\hline IReST 3 & 25 & 176.3 & 9.39 & 178.40 & 11.6 & -2.13 & 24 & -0.61 & $>.05$ & {$[-8.11,3.87]$} & 0.20 & {$[-0.35,0.76]$} \\
\hline IReST 4 & 25 & 174.9 & 9.99 & 181.55 & 15.0 & -6.62 & 24 & -1.87 & $>.05$ & {$[-12.67,-0.57]$} & 0.51 & {$[-0.05,1.07]$} \\
\hline IReST 5 & 25 & 177.2 & 10.1 & 174.81 & 12.7 & 2.42 & 24 & 0.62 & $>.05$ & {$[-4.23,9.08]$} & 0.21 & {$[-0.35,0.77]$} \\
\hline IReST 6 & 25 & 152.3 & 9.58 & 152.80 & 12.2 & -0.52 & 24 & -0.15 & $>.05$ & {$[-6.40,5.36]$} & 0.05 & {$[-0.51,0.60]$} \\
\hline IReST 7 & 25 & 174.0 & 11.5 & 175.55 & 11.5 & -1.53 & 24 & -0.53 & $>.05$ & {$[-6.51,3.45]$} & 0.13 & {$[-0.42,0.69]$} \\
\hline IReST 8 & 25 & 185.1 & 10.0 & 186.46 & 13.8 & -1.34 & 24 & -0.36 & $>.05$ & {$[-7.72,5.04]$} & 0.11 & {$[-0.45,0.63]$} \\
\hline IReST 9 & 25 & 169.7 & 9.85 & 170.55 & 13.3 & -0.87 & 24 & -0.29 & $>.05$ & {$[-6.04,4.13]$} & 0.07 & {$[-0.48,0.63]$} \\
\hline IReST 10 & 25 & 164.1 & 9.25 & 161.77 & 10.8 & 2.35 & 24 & 0.97 & $>.05$ & {$[-1.81,6.50]$} & 0.23 & {$[-0.33,0.79]$} \\
\hline
\end{tabular}




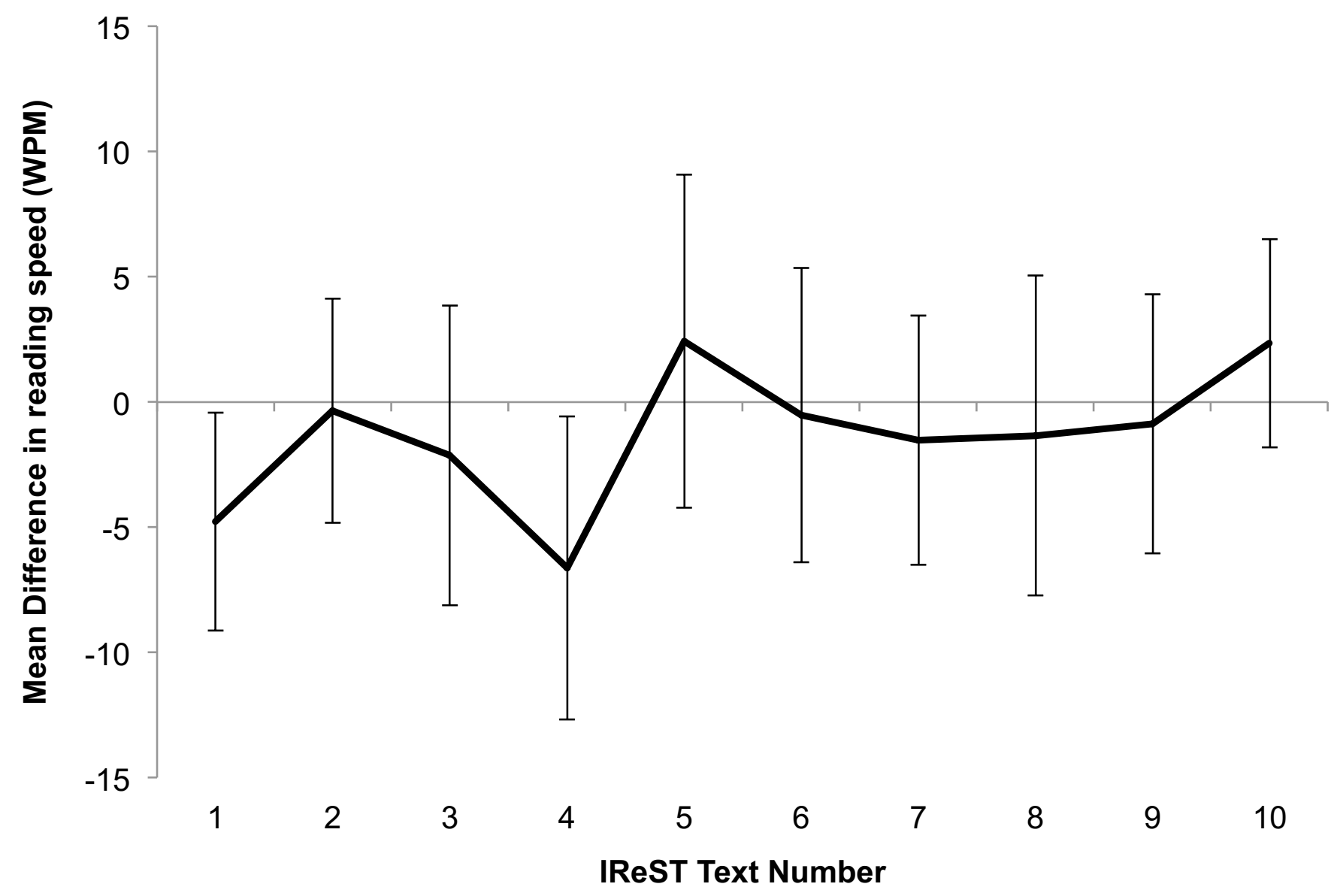

Figure 10. Mean difference in reading speeds in words per minute (WPM) between the simulated impairment conditions of the multilingual English sample and the normative values of the NA English IReST. Error bars represent 95\% confidence intervals around the mean. 
(see Figure 10). Measures of effect size using bias corrected Hedge's $g^{*}$ ranged from 0.03 to 0.51 , with $95 \%$ confidence intervals ranging from -.52 to 1.07. Further Bayesian analyses revealed $\mathrm{BF}_{10}$ factors ranging from 0.01 to 0.53 , indicating substantial evidence for the null hypothesis (see Table 12). Therefore, multilingual English speaking participants in the simulated impairment condition did not read the IReST significantly slower than L1 English speaking participants in the NA IReST.

\section{Discussion}

The purpose of this study was to determine whether the normative values of the NA English IReST are robust enough to reliably account for differences in language fluency when assessing a sample of multilingual English speaking North Americans. Conflicting results, found that there is not enough evidence to definitively state that there is no difference, or that there is a difference between reading speeds of multilingual English speakers on the IReST when compared to the normative values of the NA IReST.

There are three potential explanations for the conflicting results of this study; the first is that the sample consisted of 30\% L1 English speakers, and their reading speeds on the IReST may be negatively skewing the results thereby increasing the mean reading speed of the sample. If this were the case, then by assessing a sample of only L2 and L3 English speakers on the IReST then this would result in significantly lower reading speeds on the IReST compared to the normative NA IReST values. Alternatively, a second explanation is that as $30 \%$ of the sample consisted of L3 English speakers, their reading speeds on the IReST may be positively skewing the results such that they are lowering the mean reading speed of the sample. If this were the case, then by assessing a sample of only L1 and L2 English speakers on the IReST then this would result in no significant differences in reading speeds on the IReST compared to the normative NA IReST values. A third explanation for the conflicting results is the high English language fluency of the sample, as $94 \%$ of participants reported that they were either fluent or very good English readers. This high English language fluency may be due to the sample being drawn from an English speaking University, thus students would be required to be proficient in the English language to succeed in their studies. However, if this were the case then these results would be consistent with the findings of Gasquoine et al. (2007) who found that there were no significant effects of language when assessing balanced, or highly fluent, bilinguals. This would mean that the IReST is robust enough to account for differences in language background and the 
Table 12.

Results of the Bayesian Analysis of Reading Speeds on the IReST of the Simulated Impairment Conditions of the Multilingual English Sample and the Normative Values of the NA English IReST.

\begin{tabular}{cccccc}
\hline & \multicolumn{2}{c}{ BF } & & \multicolumn{2}{c}{ Effect Size $(\delta)$} \\
\cline { 2 - 3 } \cline { 5 - 6 } IReST 1 & $\mathrm{BF}_{10}$ & error $\%$ & & Median & CI \\
IReST 2 & 0.081 & $5.990 \times 10^{-7}$ & & 0.06 & {$[0.002,0.25]$} \\
IReST 3 & 0.192 & $2.888 \times 10^{-4}$ & & 0.12 & {$[0.01,0.40]$} \\
IReST 4 & 0.142 & $3.280 \times 10^{-4}$ & & 0.09 & {$[0.004,0.35]$} \\
IReST 5 & 0.081 & $6.009 \times 10^{-7}$ & & 0.06 & {$[0.002,0.26]$} \\
IReST 6 & 0.363 & $3.301 \times 10^{-4}$ & & 0.17 & {$[0.01,0.50]$} \\
IReST 7 & 0.189 & $2.896 \times 10^{-4}$ & & 0.12 & {$[0.01,0.41]$} \\
IReST 8 & 0.148 & $3.192 \times 10^{-4}$ & & 0.10 & {$[0.004,0.36]$} \\
IReST 9 & 0.164 & $3.029 \times 10^{-4}$ & & 0.10 & {$[0.01,0.372]$} \\
IReST 10 & 0.172 & $2.971 \times 10^{-4}$ & 0.11 & {$[0.01,0.40]$} \\
\hline
\end{tabular}


mean reading speeds of a sample of multilingual English speakers in the normal vision condition would not fall below the normative values of the NA English IReST. Generally, the results of the NHST found that there were no significant differences between the reading speeds of multilingual English speakers and the normative values of the NA IReST. The exception to this statement were for texts 5,7 , and 10 where $p$-value's were $<.03$, with a mean effect size of 0.19 . However, the Bayesian analysis revealed that there was only substantial evidence for no difference for texts 4 and 6, whereas there was only anecdotal evidence of no difference for texts 1 and 2. Moreover, there was anecdotal evidence for a difference in reading speeds between the multilingual sample and the normative values of the NA IReST on texts 3,8, 9, and 10, and substantial evidence for a difference between reading speeds on texts 5 and 7 . These results suggest that assessing an individual in their non-dominant but fluent language would represent a minor deviation in standardization protocols. Although the results of the NHST and Bayesian analyses are conflicting, one factor that may indicate that this third option may be more plausible is that all $95 \%$ confidence intervals around the mean reading speed of each text overlap (see Figure 11).

In the simulated impairment condition, the results of the NHST and the Bayesian analysis were consistent with one another, such that they both showed there were no significant differences in reading speed between the sample of multilingual English speakers and the NA IReST values. It was hypothesized that the sample of multilingual English speakers would have significantly slower reading speeds compared to the normative values of the NA IReST. Again, this may be attributable to the high proportion of participants (94\%) who reported themselves as being either fluent or very good English readers. These results may also support the hypothesis that the IReST is robust enough to account for differences in language fluency when individuals are highly fluent bilinguals.

One of the limitations of this study is that the sample consisted of L1, L2, and L3 English speakers, as opposed to comparing the normative values of the NA English IReST to a sample consisting of only L2 or only L3 English speakers. Future studies should compare the reading speeds of only L2 or L3 English speakers to determine if the IReST is robust enough to account for these differences in language background. Alternatively, the impact of language fluency on the IReST can be examined using a paradigm similar to that used by Gasquoine et al. (2007), for example, by separating the sample into groups of English-dominant bilinguals, balanced 


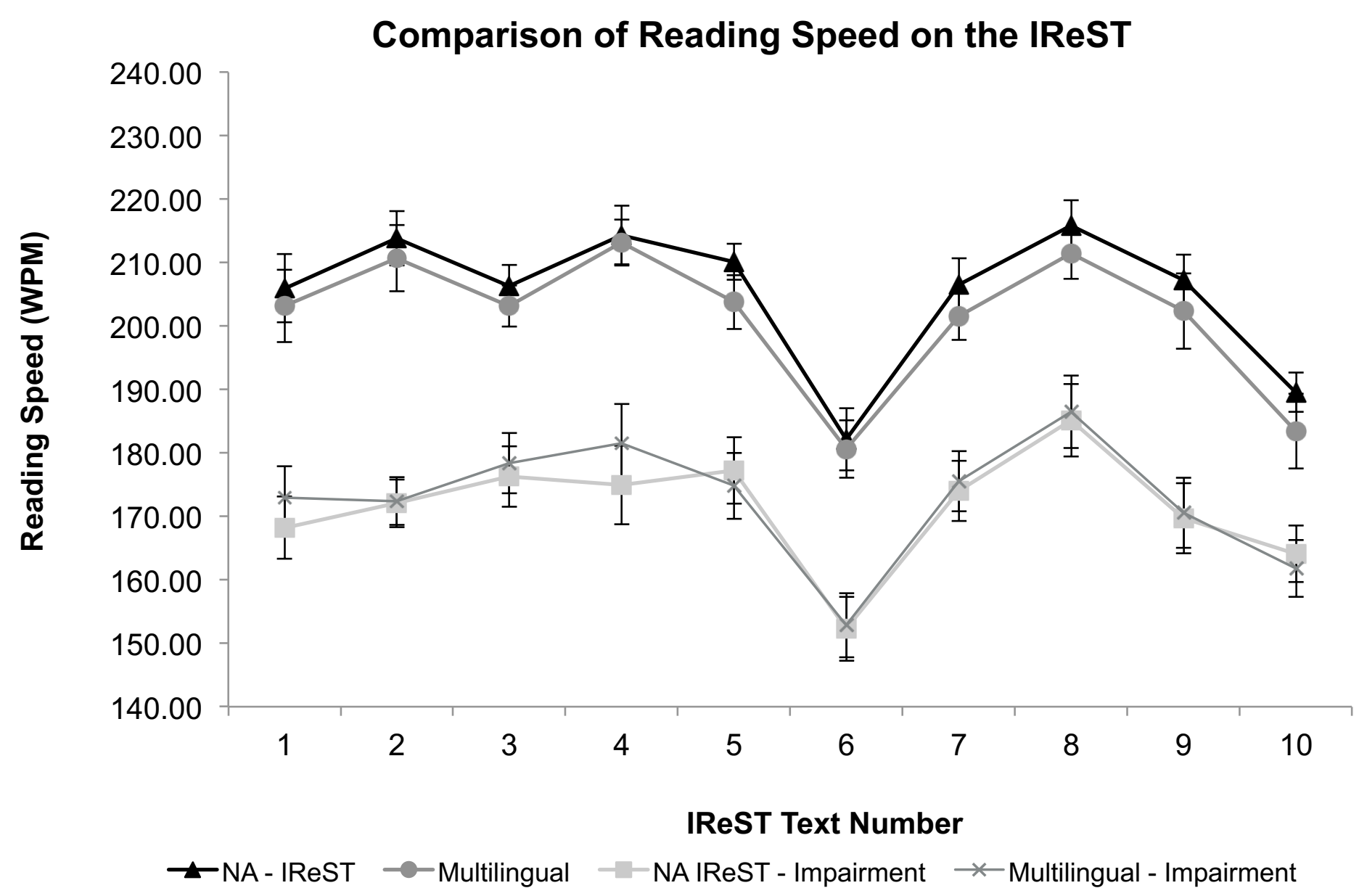

Figure 11. Mean reading speeds in words per minute (WPM) across IReST texts. Error bars represent $95 \%$ confidence intervals around the mean. 
bilinguals, and French-dominant bilinguals. It should be noted, however, while the use of a multilingual sample is a limitation, the reading speeds of this sample may be more realistic compared to those that would be observed in low vision rehabilitation settings in Quebec. A secondary analyses of the demographic data of Overbury \& Wittich (2011) reveals that 31\% of clients seeking low vision rehabilitation in Montreal report that their first language is English, $41 \%$ report that it is French, and 28\% report that it is neither English nor French. In this study $30 \%$ of participants reported that their first language was English, 40\% reported that it was French, and 30\% reported that it was neither English nor French. Thus, our sample would seem to reflect the demographics of the population that would be tested with the IReST.

The aim of Experiment 2 was to determine whether the normative values of the NA English IReST could reliably account for differences in language fluency in multilingual English speaking North Americans. While the results of the NHST and Bayesian analyses were inconsistent, and further research should be done, it would appear that the IReST may be robust enough to account for differences in language background when there is high language fluency. Future research should examine whether there are true differences in reading speeds using a sample consisting of only L2 or L3 English speakers and determine whether the results are consistent with the findings of this study. As it appears that the IReST may be able to account for minor deviations in procedures, i.e., assessing non-dominant language but highly fluent individuals on the assessment, Experiment 3 will determine whether the IReST is robust enough to account for extensive deviations in IReST standardization procedures. 


\section{Experiment 3}

The purpose of Experiment 1 was to validate the IReST in a NA sample, whereas the purpose of Experiment 2 was to determine whether language background and fluency significantly impacted reading speeds on the IReST. While the results of Experiment 2 were inconsistent, it would appear that administering the IReST to individuals in a non-dominant yet highly fluent language did not significantly impact reading speeds on the majority of IReST texts. Furthermore, assessing an individual on the IReST in their non-dominant yet highly fluent language may constitute a minor deviation in standardization protocols. The purpose of Experiment 3 is to determine whether the IReST is robust enough to account for extensive deviations in standardization protocols by administering the IReST to a sample of multilingual English speakers while not following the IReST administration procedures; i.e., not instructing participants to read the texts aloud as quickly as possible and without going back and making corrections. It is hypothesized that improper administration of the IReST will negatively impact reading speed in both the normal vision and simulated impairment conditions.

In a study by Sattler (1969), it has been shown that administering assessments using improper administration protocols can significantly impact test takers performance. The study examined the impact of single versus multiple administrator cues on the Block Design and Picture Arrangement subtests of the Wechsler Intelligence Scale for Children. The results showed that a single administrator cue did not significantly impact individual's performance on the assessment, however, multiple administrator cues did significantly increase participants scores. Therefore, Sattler (1969) concludes that minor alterations in standardized procedures, i.e., one administrator cue, are less likely to impact the results of an assessment compared to extensive alterations to standardization procedures, i.e., multiple administrator cues.

As it has been shown that language of test administration on the IReST may be a minor deviation in standardization procedure, this study will examine whether ignoring administration protocols would be an extensive deviation in standardization procedure. In low vision rehabilitation settings, improper administration of assessments, such as the IReST, may be more likely to occur if assessors have not been formally trained on the assessment. A study by Pell, Homer, and Roberts (2008) examined whether trained versus untrained assessment administrators impacted test-takers performance on the assessments. The results showed that there were significant differences in the evaluations of trained versus untrained assessment 
administrators, such that untrained assessors inflated the scores of test-takers. Therefore, it is crucial to examine the impact of improper administration of the IReST to determine whether or not this could impact the rehabilitation outcomes of low vision rehabilitation clients; e.g., if improper administration of the IReST results in significantly reduced reading speeds, clients may be diagnosed with a reading disorder or a mild cognitive impairment (Lebowitz et al., 2015)

Experiment 3 will examine the impact of improper administration of the IReST to determine: (1) whether the reading speeds of a sample of multilingual English speakers will fall outside of the normative values of the NA English IReST when administration protocols are not followed; and (2) the impact of a simulated sensory impairment on reading speed when the IReST administration protocols are not followed. In this experiment, normally sighted multilingual English speakers will be asked to read all 10 English language IReST texts aloud with no further instructions. It was hypothesized that; (1) the mean reading speeds of this sample will fall below the normative values of the NA English IReST; and (2) that the simulated visual impairments would further reduce mean reading speeds across texts.

\section{Method}

The research protocol was approved by the research ethics board of Concordia University (certificate 30003975), in accordance with the Canadian Tri-Council Policy Statement of ethical conduct for research involving humans. Experiment 3 used a slightly different method than was used in Experiments 1 and 2, however, the same measures and materials were used.

\section{Participants}

Fifty undergraduate students $(n=50)$ between the ages of 19 and $43(M=23.80, S D=4.42)$ were recruited from Concordia University to participate in this study. Participants were recruited using the Concordia University Psychology Participant Pool and were granted course credit for participating. Participants were required to have normal, or corrected-to-normal, vision. See Table 13 for participant demographics.

\section{Procedure}

The same procedure that was used in experiments 1 and 2 was used in experiment 3; however, the IReST administration protocol was not followed in this experiment. More specifically, participant were told to simply read the texts aloud as opposed to being told to read the text out-loud as quickly and as possible without going back and making corrections. 
Table 13.

Participant demographics

\begin{tabular}{|c|c|c|}
\hline Demographics & $n$ & $\%$ \\
\hline Total Survey Respondents $(n=50)$ & & $100 \%$ \\
\hline Male & 11 & $22 \%$ \\
\hline Female & 39 & $78 \%$ \\
\hline \multicolumn{3}{|l|}{ Age } \\
\hline $18-25$ & 37 & $74 \%$ \\
\hline $26-35$ & 11 & $22 \%$ \\
\hline $36-45$ & 2 & $4 \%$ \\
\hline \multicolumn{3}{|l|}{ First Language } \\
\hline English & 27 & $54 \%$ \\
\hline French & 8 & $16 \%$ \\
\hline Other & 15 & $30 \%$ \\
\hline \multicolumn{3}{|l|}{ Second Language $(n=48)$} \\
\hline English & 18 & $37.50 \%$ \\
\hline French & 22 & $45.83 \%$ \\
\hline Other & 8 & $16.67 \%$ \\
\hline \multicolumn{3}{|l|}{ Third Language $(n=28)$} \\
\hline English & 1 & $3.6 \%$ \\
\hline French & 9 & $32.1 \%$ \\
\hline Other & 18 & $64.3 \%$ \\
\hline \multicolumn{3}{|c|}{ Visual impairment not corrected through lenses } \\
\hline Yes & 1 & $2 \%$ \\
\hline No & 49 & $98 \%$ \\
\hline \multicolumn{3}{|l|}{ Known hearing impairment } \\
\hline Yes & 0 & $0 \%$ \\
\hline No & 50 & $100 \%$ \\
\hline \multicolumn{3}{|l|}{ Reading/Attention Disability } \\
\hline Yes & 2 & $4 \%$ \\
\hline No & 48 & $96 \%$ \\
\hline
\end{tabular}




\section{Results}

\section{Data Analysis}

As in the previous experiments, the data in Experiment 3 were combined to create 25 meta-participants for the normal and impaired vision conditions and participants between-subject variability was removed. A Bayesian two-sample dependent (or paired) t-test was used to determine: (1) whether the low vision simulator goggles impaired participant's visual acuity and contrast sensitivity to the same degree throughout the experiment; (2) whether the reading speeds of participants in the improper administration group (IAG) fell below the normative values of the NA IReST; and (3) whether the reading speeds of the IAG wearing in the impairment condition fell below the normative values of the simulated impairment condition of the NA IReST. Bayes factors (BF) were calculated using JASP 0.8.0.1 (JASP Team, 2016) and for each analysis a Cauchy prior width of 0.707 was used. For each analysis, the robustness of the posterior was checked using wider priors of 1.00 and 1.50 to ensure that the choice of prior distribution did not significantly impact the results. As in the previous experiments the results here are reported as both traditional NHST and Bayesian two-sample dependent t-tests, along with measures of effect sizes and confidence intervals so that the reader may interpret their statistic of choice.

\section{Descriptive Statistics}

The majority of participants (54\%) reported that their first language was English, whereas $81.2 \%$ of participants reported that their dominant language was English. Eighty-eight percent of L1 English participants reported they were fluent English readers ( $m=4.88, s d=.32), 87.5 \%$ of L1 French participants reported they were fluent English readers $(m=4.88, s d=.35)$, and 66\% of L1 Other participants reported they were fluent English readers $(m=4.66, s d=.49)$. All participants (100\%) were bilingual, and $70 \%$ of participants were trilingual. See Table 14 for a breakdown of participants self-reported language fluency (speaking, reading, writing, and listening ability) in English, French, and third languages.

In the normal vision condition, $81.6 \%$ of the reading comprehension questions were answered correctly and in the simulated impairment condition $86 \%$ of the questions were answered correctly. See Appendix D for a breakdown of the proportion of questions answered correctly for each of the texts. Similar to what occurred in the previous experiments, it appeared that participants experienced difficulty answering the reading comprehension question for text 2 , and again it is believed that this difficulty reflects the poor quality of the question. 
Table 14.

Language Fluency

\begin{tabular}{|c|c|c|c|c|c|c|c|}
\hline L1 & Language Ability & $n$ & No Ability & Elementary & Moderate & Very Good & Fluent \\
\hline \multirow[t]{5}{*}{$\begin{array}{l}\text { English } \\
(n=15)\end{array}$} & English & 27 & & & & & \\
\hline & Speaking & & 0 & 0 & 0 & 2 & 25 \\
\hline & Reading & & 0 & 0 & 0 & 3 & 24 \\
\hline & Writing & & 0 & 1 & 0 & 1 & 25 \\
\hline & Listening & & 0 & 0 & 0 & 1 & 26 \\
\hline
\end{tabular}

French 26

Speaking

Reading

Writing

Listening

Other

14

Speaking

Reading

Writing

Listening

French

English

$3 \quad 5$

0

6

4

3

4

4

8

3

9

$6 \quad 10$

1

9

8

2

11

\section{Speaking \\ Reading \\ Writing \\ Listening}

French

Speaking

Reading

Writing

Listening

8

Other

Speaking

Reading

Writing

Listening

$\begin{array}{ll}2 & 2 \\ 2 & 1 \\ 4 & 3 \\ 1 & 3\end{array}$

3
3
2
1

2

1

1

5

$\begin{array}{lllll}0 & 0 & 0 & 2 & 6 \\ 0 & 0 & 0 & 1 & 7 \\ 0 & 0 & 0 & 2 & 6 \\ 0 & 1 & 0 & 1 & 6\end{array}$

8

$\begin{array}{lllll}0 & 0 & 1 & 1 & 6 \\ 0 & 0 & 0 & 1 & 7 \\ 0 & 0 & 1 & 0 & 7 \\ 0 & 1 & 0 & 0 & 7\end{array}$

6 
Table 14. cont.

Language Fluency

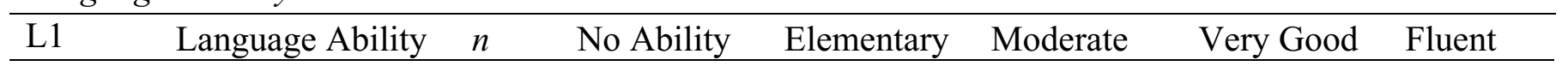

Other

$(n=15) \quad$ English

15

Speaking

Reading

Writing

Listening

French

Speaking

Reading

Writing

Listening

Other

Speaking

Reading

Writing

Listening

0
0
0
0

$\begin{array}{cccc}0 & 0 & 6 & 9 \\ 0 & 0 & 5 & 10 \\ 2 & 0 & 4 & 9 \\ 0 & 0 & 3 & 12\end{array}$

15

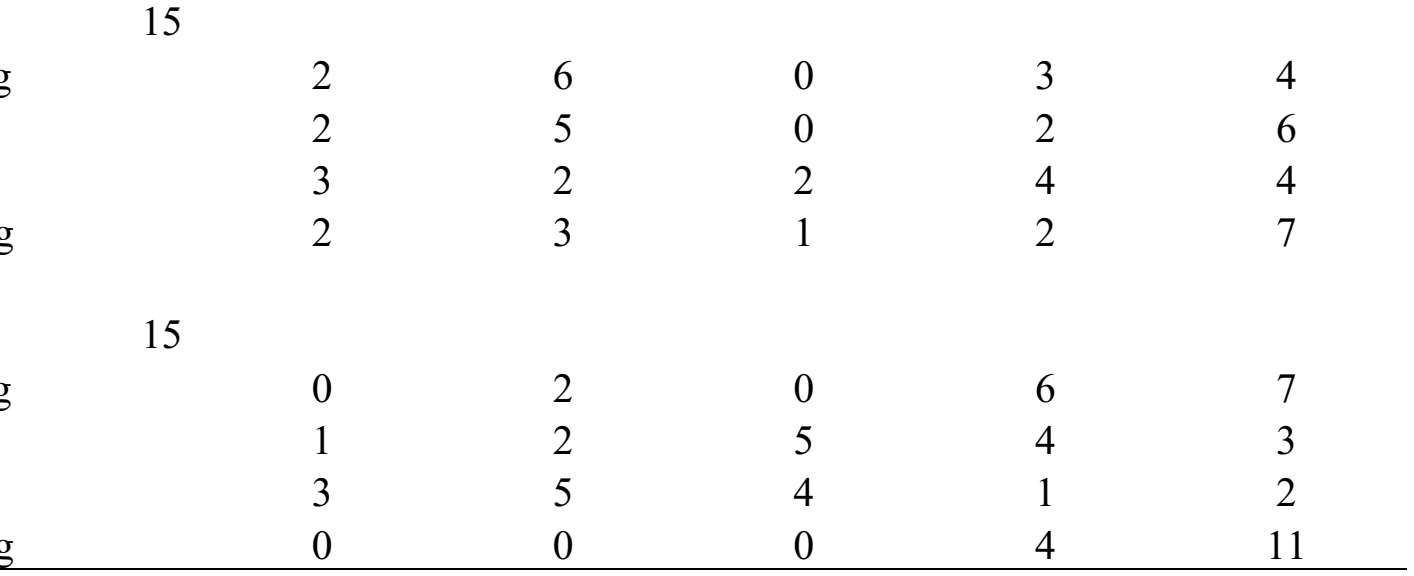

15 


\section{Visual Acuity and Low Vision Simulator Goggles}

Participants' visual acuity and contrast sensitivity were measured at three time points: (1) at baseline without the simulator goggles in the normal vision condition, (2) before reading the texts with the simulator goggles in the impaired condition, and (3) after reading the texts with the simulator goggles in the impaired condition. At the first time-point, participants had normal, or corrected to normal, vision, with an average visual acuity of $-0.10 \log \operatorname{MAR}(M=-0.10, S D=0.08$, $C I[-0.12,-0.08])$ and an average contrast sensitivity of $1.95 \log C S(M=1.95, S D=0.59, C I[1.68$, 2.12]), see Figure 12. The low vision simulator goggles were successful at both the second and third time-points at simulating a 20/80 (0.60 logMAR) visual impairment. At the second timepoint the goggles simulated a visual acuity of $0.59 \log \operatorname{MAR}(M=0.59, S D=0.19, C I[0.54,0.64])$ and contrast sensitivity of $.95 \log C S(M=.95, S D=.59, C I[0.88,1.02])$, and at the third timepoint the goggles simulated a visual acuity of $0.57 \log \operatorname{Mar}(M=0.57, S D=0.19, C I[0.52,0.63])$ and contrast sensitivity of $0.99 \log C S(M=0.99, S D=0.22, C I[0.93,1.05])$. There were no significant differences between the participants visual acuity at the second and third time-points , $t(49)=0.84, p>.05, d=0.12, C I[-0.03,0.08]$; further Bayesian analysis revealed a $\mathrm{BF}_{01}$ factor of 4.65 , indicating that the probability of the null hypothesis is 4.65 times greater than the probability of the research hypothesis. There were also no significant differences between the participants contrast sensitivity at the second and third time points, $t(49)=-1.19, p>.05, d=$ $0.17, C I[-0.10,0.03]$; further, Bayesian analysis revealed a $\mathrm{BF}_{01}$ factor of 3.34, indicating that the probability of the null hypothesis is 3.34 times greater than the probability of the research hypothesis. Therefore, participants received the same degree of simulated visual impairment across the impairment condition.

\section{IAG Compared to the Impaired Normative NA IReST Values}

To test the hypothesis that the reading speeds of the IAG would fall below the normative values established in the normal vision condition of the NA IReST, mean reading speeds in wpm were calculated for each text (see Table 15). Multiple two-sample dependent t-tests using HolmBonferonni corrections for multiple comparisons were used to compare the mean reading speed in wpm of the IAG and NA IReST in the normal vision condition; the results showed statistically significant differences between the mean reading speeds of the IAG and the NA IReST values (see Table 15). In all cases $p$-values were less than 0.001. Mean difference scores ranged from $20.41 \mathrm{wpm}$ to $26.52 \mathrm{wpm}$, with $95 \%$ confidence intervals ranging from 15.65 to 30.74 (see 

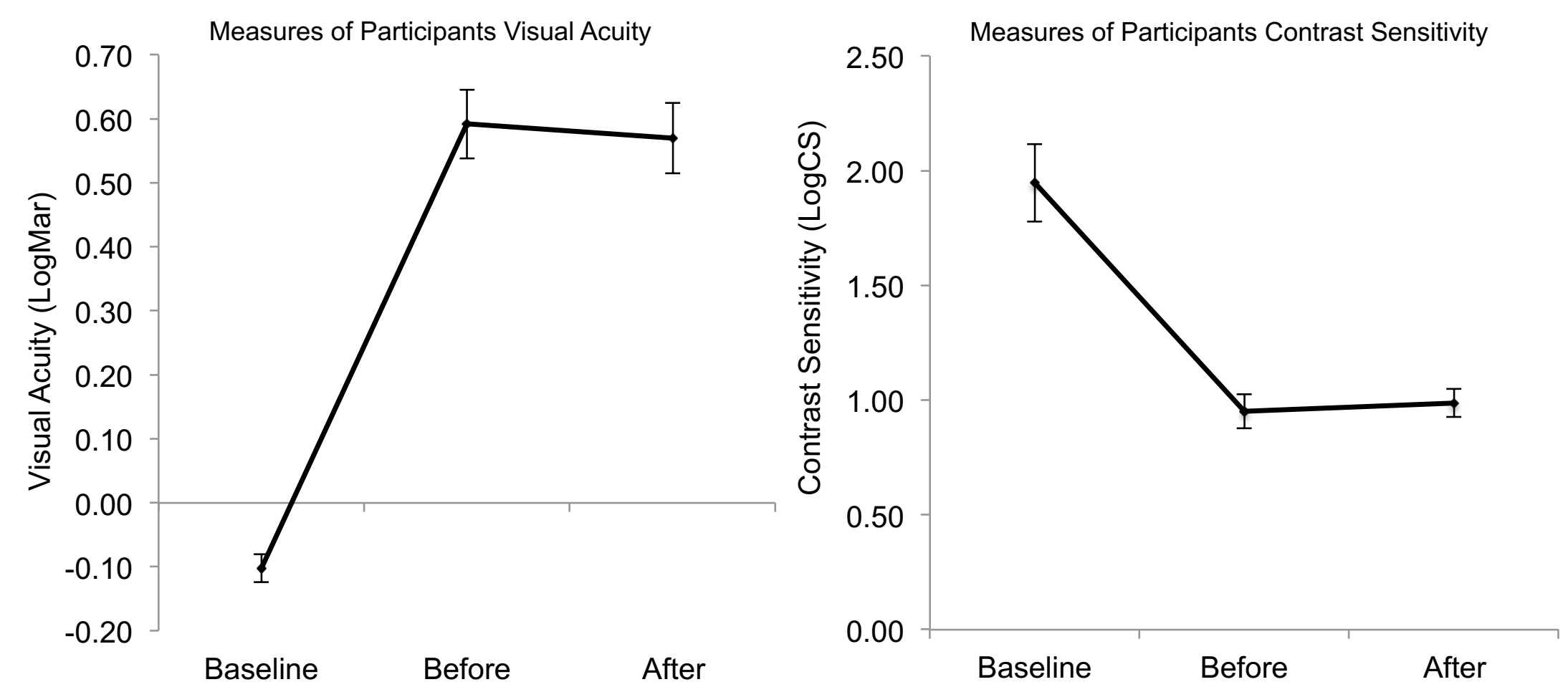

Figure 12. Measures of participant's visual acuity and contrast sensitivity. These figures represent changes in participant's visual acuity and contrast sensitivity from baseline to before and after the simulated visual impairment conditions. Error bars represent $95 \%$ confidence intervals around the mean. 
Table 15 .

Results of the Two Sample Dependent t-test Between Reading Speeds on the IReST of the Normative Values of the NA English IReST and the IAG Normal Vision Condition.

\begin{tabular}{|c|c|c|c|c|c|c|c|c|c|c|c|c|}
\hline & \multirow[b]{2}{*}{$\mathrm{n}$} & \multicolumn{2}{|c|}{$\begin{array}{c}\text { NA IReST - } \\
\text { Normal }\end{array}$} & \multicolumn{2}{|c|}{ IAG - Normal } & \multicolumn{7}{|c|}{ Dependent Samples t-test } \\
\hline & & $m$ & $s d$ & $m$ & $s d$ & Mdiff & $d f$ & $t$ & $p$ & $C I$ & $\begin{array}{c}\text { Hedge's } \\
g^{*}\end{array}$ & $C I$ \\
\hline IReST 1 & 25 & 205.9 & 13.0 & 182.24 & 8.22 & 23.71 & 24 & 9.07 & $<.001$ & {$[19.24,28.19]$} & 2.16 & {$[1.46,2.86]$} \\
\hline IReST 2 & 25 & 213.8 & 10.4 & 187.34 & 9.29 & 26.52 & 24 & 10.74 & $<.001$ & {$[22.29,30.74]$} & 2.66 & {$[1.90,3.42]$} \\
\hline IReST 3 & 25 & 206.3 & 7.9 & 183.25 & 8.89 & 23.07 & 24 & 13.49 & $<.001$ & {$[20.15,26.00]$} & 2.71 & {$[1.95,3.48]$} \\
\hline IReST 4 & 25 & 214.3 & 11.1 & 190.27 & 9.29 & 24.05 & 24 & 7.75 & $<.001$ & {$[18.73,29.36]$} & 2.31 & {$[1.60,3.03]$} \\
\hline IReST 5 & 25 & 210.1 & 6.9 & 185.28 & 8.32 & 24.87 & 24 & 14.45 & $<.001$ & {$[21.93,27.82]$} & 3.22 & {$[2.38,4.06]$} \\
\hline IReST 6 & 25 & 182.1 & 11.8 & 161.72 & 7.81 & 20.41 & 24 & 7.33 & $<.001$ & {$[15.65,25.18]$} & 2.02 & {$[1.33,2.70]$} \\
\hline IReST 7 & 25 & 206.5 & 10.0 & 181.19 & 6.45 & 25.31 & 24 & 12.33 & $<.001$ & {$[21.80,28.83]$} & 2.97 & {$[2.17,3.78]$} \\
\hline IReST 8 & 25 & 215.8 & 9.6 & 192.70 & 11.00 & 23.12 & 24 & 8.35 & $<.001$ & {$[18.38,27.86]$} & 2.21 & {$[1.51,2.91]$} \\
\hline IReST 9 & 25 & 207.2 & 9.7 & 181.80 & 8.41 & 25.48 & 24 & 9.80 & $<.001$ & {$[21.03,29.93]$} & 2.78 & {$[2.00,3.56]$} \\
\hline IReST 10 & 25 & 189.5 & 7.4 & 168.52 & 9.83 & 21.02 & 24 & 9.98 & $<.001$ & {$[17.42,24.62]$} & 2.38 & {$[1.66,3.11]$} \\
\hline
\end{tabular}


Figure 13). Measures of effect size using bias corrected Hedge's $g^{*}$ ranged from 2.02 to 3.221 , with $95 \%$ confidence intervals ranging from 1.33 to 4.06. Further Bayesian analyses revealed $\mathrm{BF}_{10}$ factors ranging from $215,247.02$ to $5.776 \times 10^{10}$, indicating decisive evidence for the research hypothesis (see Table 16). Thus, participants in the normal vision condition in the IAG read the IReST significantly slower than the normative values established in the NA IReST normal vision condition.

\section{Impaired IAG Compared to the Impaired Normative NA IReST Values}

To test the hypothesis that the reading speeds of the impaired IAG would fall below the normative values established in the simulated visual impairment condition of the NA IReST, mean reading speeds in wpm were calculated for each text (see Table 17). Multiple two-sample dependent t-tests using Holm-Bonferonni corrections for multiple comparisons were used to compare the mean reading speed in wpm of impaired IAG and the impaired NA IReST condition; the results showed statistically significant differences between the mean reading speeds of the impaired IAG and the normative values established in the NA IReST simulated visual impairment condition (see Table 17). In all cases $p$-values were less than 0.001. Mean difference scores ranged from $14.71 \mathrm{wpm}$ to $26.81 \mathrm{wpm}$, with $95 \%$ confidence intervals ranging from 9.21 to 32.55 (see Figure 14). Measures of effect size using bias corrected Hedge's $g$ * ranged from 1.45 to 2.43 , with $95 \%$ confidence intervals ranging from 0.83 to 3.16 . Further Bayesian analyses revealed $\mathrm{BF}_{10}$ factors ranging from 4.551 to $3.442 \times 10^{6}$, indicating substantial evidence for the null hypothesis (see Table 18). Therefore, participants in the simulated visual impairment condition in the IAG read the IReST significantly slower than the normative values established in the NA IReST simulated impairment condition.

\section{Discussion}

The purpose of this study was to determine the impact of not following IReST administration protocols on reading speeds on the IReST. It was hypothesized that the mean reading speeds of participants in the IAG group in the normal vision condition would fall below the normative values of the NA English IReST. The results showed that there were significant differences between the reading speeds of the normal vision IAG group and the normative values of the NA English IReST. More specifically, administering the IReST with the improper administration protocols decreased participants reading speeds across all 10 texts by an average of 24 wpm (Figure 15). There were also significant differences between reading speeds in the 


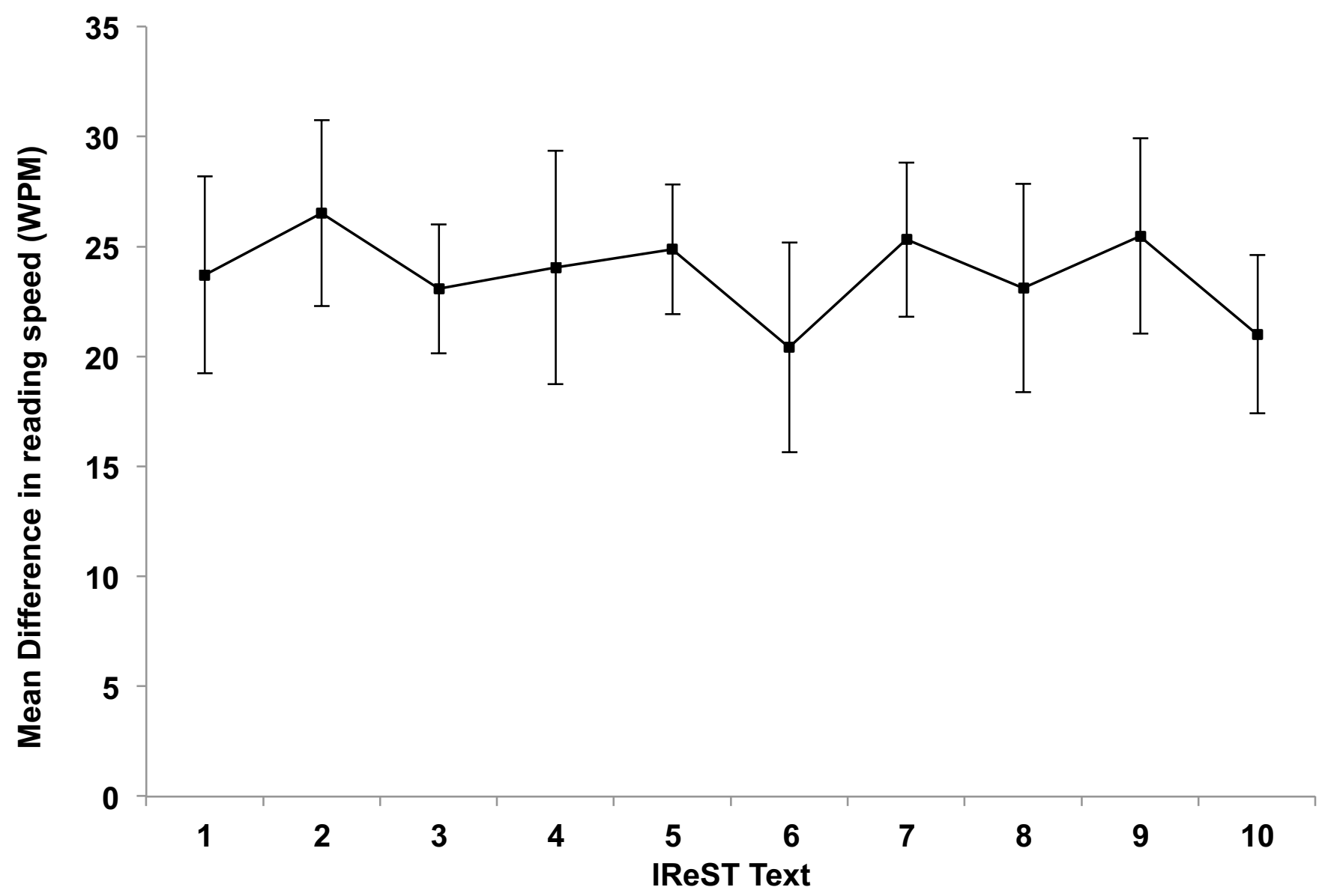

Figure 13. Mean difference in reading speeds in words per minute (WPM) between the normative values of the NA English IReST and the IAG normal vision condition. Error bars represent $95 \%$ confidence intervals. 
Table 16.

Results of the Bayesian Analysis of Reading Speeds on the IReST of the Normative Values of the NA English IReST and the IAG Normal Vision Condition.

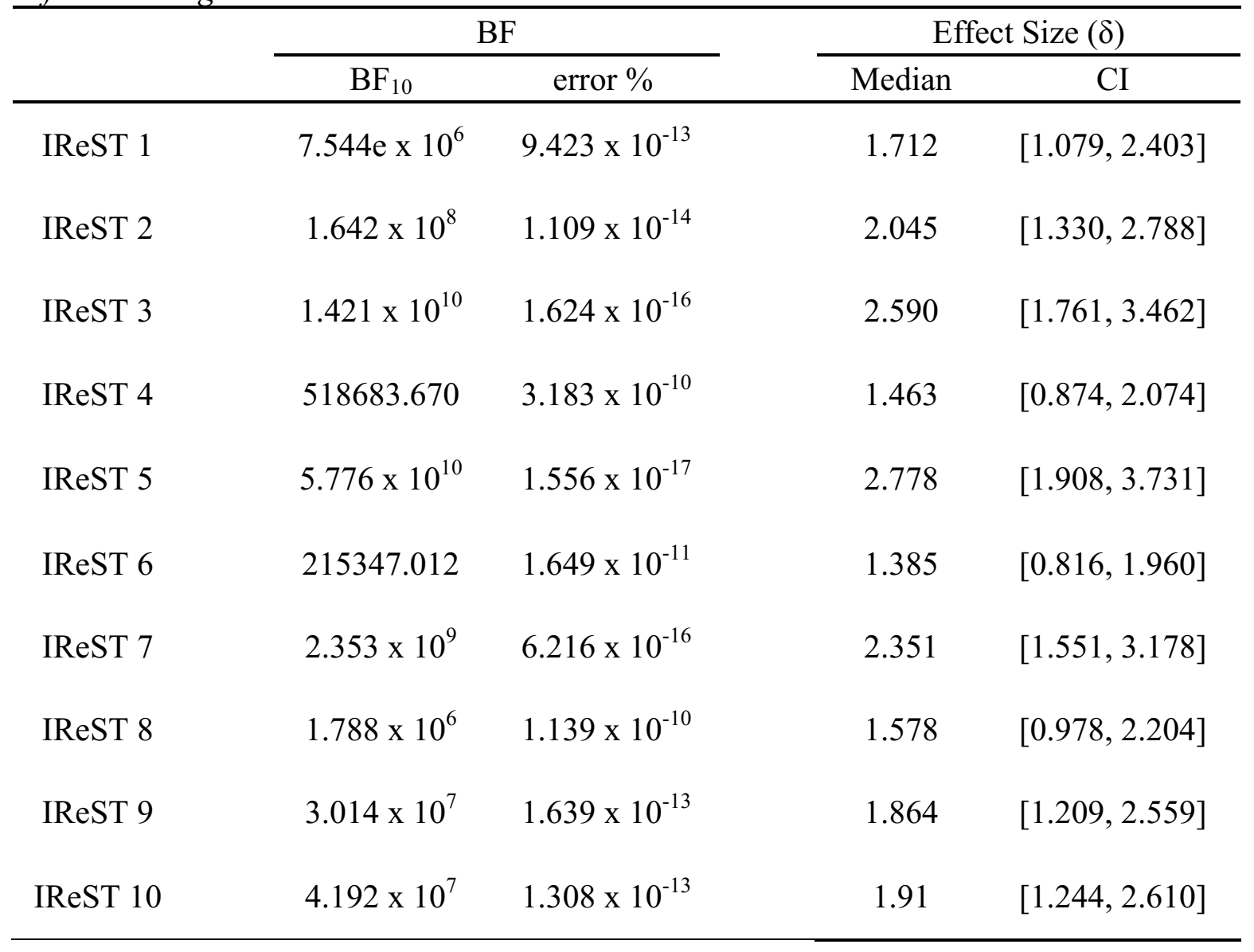


Table 17.

Results of the Two Sample Dependent t-test Between Reading Speeds on the IReST of the Simulated Impairment Condition of the Normative Values of the NA English IReST and the IAG Simulated Impairment Condition

\begin{tabular}{|c|c|c|c|c|c|c|c|c|c|c|c|c|}
\hline & \multirow[b]{2}{*}{$\mathrm{n}$} & \multicolumn{2}{|c|}{$\begin{array}{l}\text { NA IReST - } \\
\text { Impaired }\end{array}$} & \multicolumn{2}{|c|}{ IAG - Impaired } & \multicolumn{7}{|c|}{ Dependent Samples t-test } \\
\hline & & $m$ & $s d$ & $m$ & $s d$ & Mdiff & $d f$ & $t$ & $p$ & $C I$ & $\begin{array}{l}\text { Hedge's } \\
g^{*}\end{array}$ & $C I$ \\
\hline IReST 1 & 25 & 168.2 & 7.53 & 147.14 & 14.8 & 21.08 & 24 & 7.458 & $<.001$ & {$[16.24,25.91]$} & 1.77 & {$[1.12,2.43]$} \\
\hline IReST 2 & 25 & 172.1 & 13.5 & 148.44 & 14.4 & 23.62 & 24 & 7.428 & $<.001$ & {$[18.18,29.06]$} & 1.67 & {$[1.03,2.32]$} \\
\hline IReST 3 & 25 & 176.3 & 9.39 & 152.01 & 10.4 & 24.27 & 24 & 8.540 & $<.001$ & {$[19.41,29.13]$} & 2.43 & {$[1.70,3.16]$} \\
\hline IReST 4 & 25 & 174.9 & 9.99 & 160.22 & 10.1 & 14.71 & 24 & 4.582 & $<.001$ & {$[9.21,20.20]$} & 1.45 & {$[0.83,2.07]$} \\
\hline IReST 5 & 25 & 177.2 & 10.1 & 158.39 & 14.8 & 18.84 & 24 & 5.327 & $<.001$ & {$[12.79,24.89]$} & 1.47 & {$[0.84,2.09]$} \\
\hline IReST 6 & 25 & 152.3 & 9.58 & 125.47 & 12.8 & 26.81 & 24 & 7.985 & $<.001$ & {$[21.07,32.55]$} & 2.35 & {$[1.63,3.07]$} \\
\hline IReST 7 & 25 & 174.0 & 11.5 & 151.20 & 10.5 & 22.82 & 24 & 7.636 & $<.001$ & {$[17.70,27.93]$} & 2.05 & {$[1.36,2.73]$} \\
\hline IReST 8 & 25 & 185.1 & 10.0 & 159.82 & 12.0 & 25.30 & 24 & 7.988 & $<.001$ & {$[19.88,30.72]$} & 2.26 & {$[1.55,2.97]$} \\
\hline IReST 9 & 25 & 169.7 & 9.85 & 152.03 & 11.3 & 17.65 & 24 & 8.673 & $<.001$ & {$[14.17,21.14]$} & 1.65 & {$[1.01,2.29]$} \\
\hline IReST 10 & 25 & 164.1 & 9.25 & 143.04 & 11.2 & 21.07 & 24 & 7.712 & $<.001$ & {$[16.39,25.75]$} & 2.03 & {$[1.34,2.71]$} \\
\hline
\end{tabular}




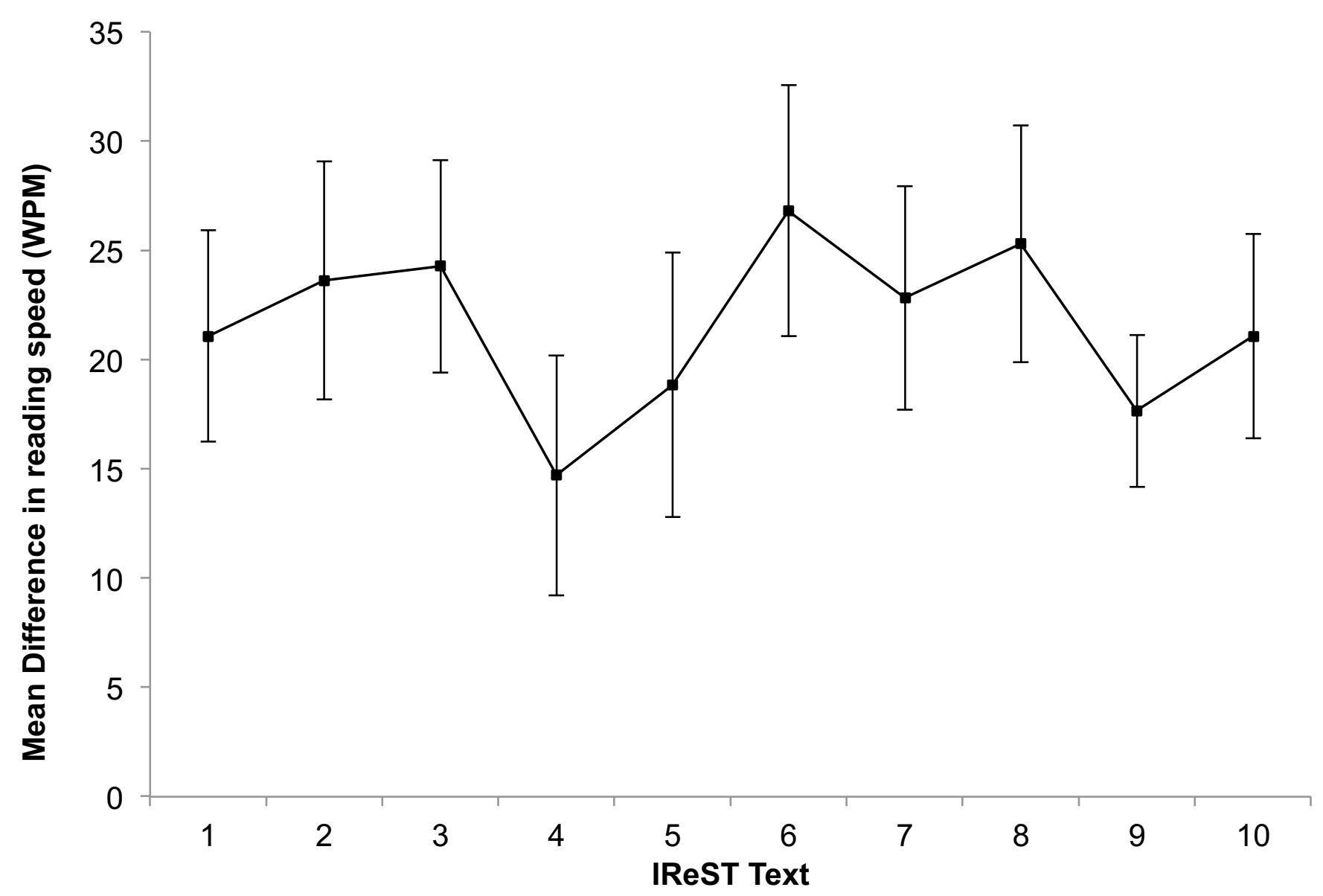

Figure 14. Mean difference in reading speeds in words per minute (WPM) between the simulated impairment condition of the normative values of the NA English IReST and the IAG simulated impairment group. Error bars represent $95 \%$ confidence intervals around the mean. 
Table 18.

Results of the Bayesian Analysis of Reading Speeds on the IReST of the Simulated Impairment Condition of the Normative Values of the NA English IReST and the IAG Simulated Impairment Condition.

\begin{tabular}{|c|c|c|c|c|}
\hline & \multicolumn{2}{|c|}{$\mathrm{BF}$} & \multicolumn{2}{|c|}{ Effect Size $(\delta)$} \\
\hline & $\mathrm{BF}_{10}$ & error $\%$ & Median & $\mathrm{CI}$ \\
\hline IReST 1 & 283236.4 & $1.645 \times 10^{-11}$ & 1.406 & {$[0.842,1.992]$} \\
\hline IReST 2 & 266207.6 & $1.659 \times 10^{-11}$ & 1.407 & {$[1.407,2.788]$} \\
\hline IReST 3 & $2.639 \times 10^{6}$ & $3.154 \times 10^{-12}$ & 1.611 & {$[0.997,2.264]$} \\
\hline IReST 4 & 455.1 & $2.142 \times 10^{-7}$ & 0.846 & {$[0.387,1.320]$} \\
\hline IReST 5 & 2506.7 & $5.002 \times 10^{-8}$ & 0.988 & {$[0.500,1.494]$} \\
\hline IReST 6 & 853956.4 & $2.827 \times 10^{-11}$ & 1.504 & {$[0.927,2.108]$} \\
\hline IReST 7 & 412696.8 & $5.883 \times 10^{-10}$ & 1.446 & {$[0.866,2.048]$} \\
\hline IReST 8 & 859430.5 & $2.670 \times 10^{-11}$ & 1.507 & {$[0.916,2.118]$} \\
\hline IReST 9 & $3.442 \times 10^{6}$ & $2.350 \times 10^{-12}$ & 1.645 & {$[1.038,2.291]$} \\
\hline IReST 10 & 484576.1 & $3.880 \times 10^{-10}$ & 1.463 & {$[0.880,2.052]$} \\
\hline
\end{tabular}




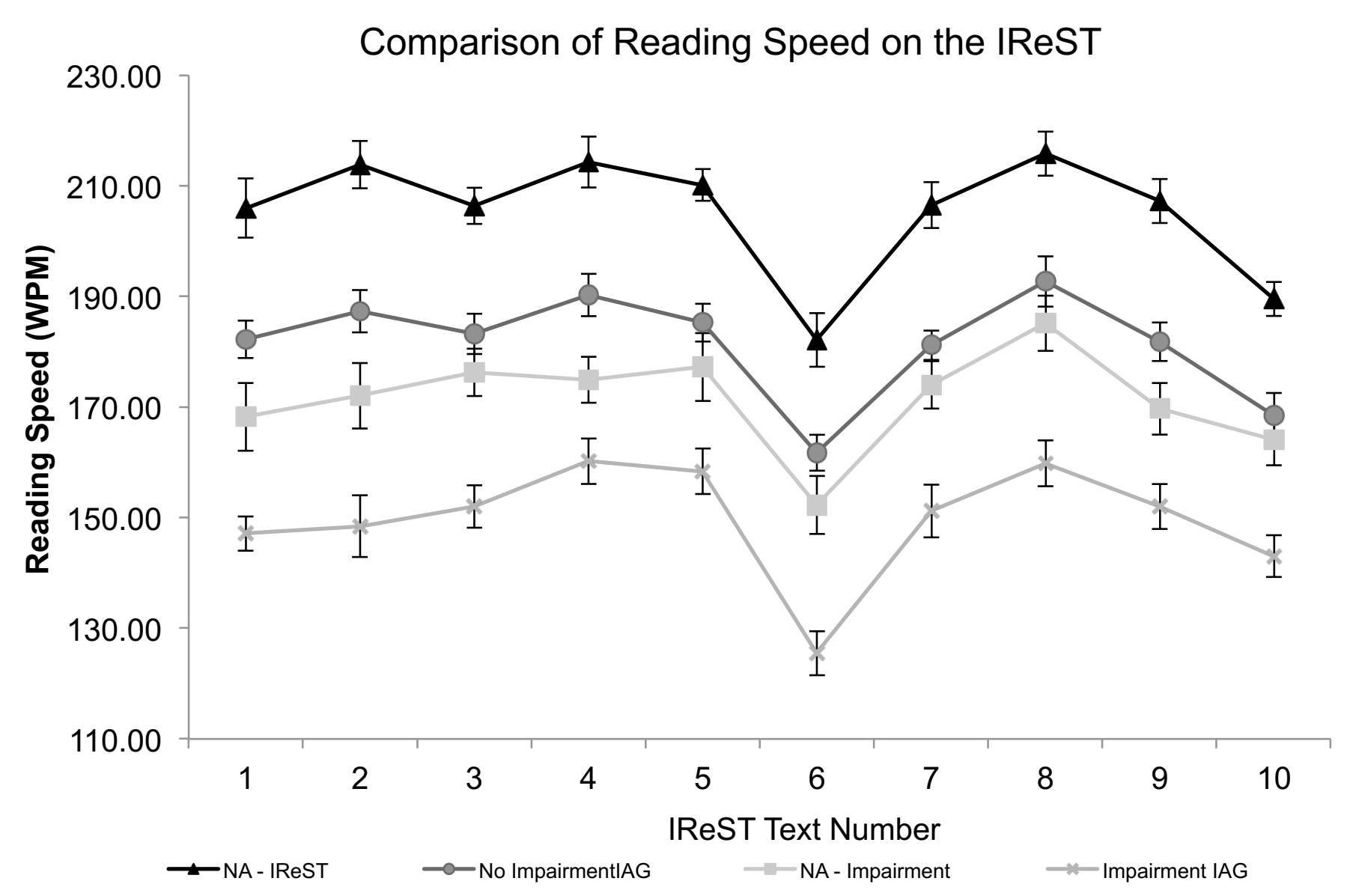

Figure 15. Mean reading speeds in words per minute (WPM) across IReST texts. Error bars represent $95 \%$ confidence intervals around the mean. 
simulated impairment IAG group compared to the reading speeds of the normative values of the simulated impairment NA English IReST. Similar to the normal vision IAG condition, in the simulated impairment IAG condition participants reading speeds across all 10 texts were decreased by an average of $22 \mathrm{wpm}$ compared to the normative values of the NA English IReST simulated impairment condition. Therefore, it would appear that not following the IReST administration protocol constitutes an extensive deviation from standardization protocols, consistent with the findings of Sattler (1969).

It is worth noting that the reading speeds in both the normal and simulated impairment IAG conditions follow the same overall pattern of results of the NA English IReST, but shifted downwards by an average of 24 and 22 wpm respectively (see Figure 15). Secondary analyses also revealed that there were significant differences between the reading speeds of the normal vision IAG group, and the reading speeds of the simulated impairment condition of the NA IReST. Thus, it would appear that the impact of the improper administration of the IReST is that it significantly reduces the reading speeds of test-takers. However, this reduction in reading speed is not as severe as the impact of a simulated visual impairment. Importantly, the results of the study show that the improper administration of the IReST, when coupled with a simulated visual impairment, results in an average reduction in reading speed of $55 \mathrm{wpm}$. As secondary analyses from Experiment 1 found that the reading speeds of the simulated impairment condition of the NA IReST are likely an underestimate of the true reading speeds of older adults with sensory impairments, it is likely that the improper administration of the IReST to a sample of visually impaired older adults would further reduce their reading speeds. Future studies should determine the impact of improper administration of the IReST on older adults with visual impairments, because, as previously mentioned, severe reductions in reading speeds may lead to a misdiagnosis of a reading disorder or mild cognitive impairment (Lebowitz et al., 2015).

A limitation of this study is that the sample consisted of a group of multilingual English speakers, and therefore it is possible that part of the reduction in reading speed is due to variability that is attributable to language background and fluency. Conversely, the results of Experiment 2 found that, for the majority of the IReST texts there were no significant differences in reading speeds between a multilingual sample of English speakers and L1 English speakers. 
Furthermore, as the $95 \%$ confidence intervals of each text in the normal and impaired vision conditions in Experiment 2 overlapped, the variability that in this experiment that is attributable to differences in language fluency and background may be negligible. However, future studies should examine the impact of the improper administration of the IReST protocols in a sample consisting solely of L1, L2, or L3 English speakers.

The aim of Experiment 3 was to determine whether the improper administration of the IReST constituted an extensive deviation in standardization procedures such that this would impact participant performance on the IReST. It was observed that not following the administration protocols of the IReST negatively impacted participant's performance in both the normal and simulated impairment conditions, such that both groups experienced significant reductions in reading speeds. To reduce the likelihood of this occurring in low vision rehabilitation settings, administrators of the IReST should carefully follow the IReST administration protocols as described on the instruction page of the IReST. Alternatively, low vision rehabilitation specialists should be trained in the IReST administration protocols to ensure that they obtain accurate measures of clients reading speeds (Pell et al., 2008). 


\section{General Discussion}

The English language IReST is a measure of reading speed that was developed and normalized in the UK. Although the IReST is used to assess reading speed in low vision rehabilitation settings throughout the US and Canada, the normative values of the IReST have never been validated in these populations. The purpose of this thesis was therefore to: (1) validate the English language IReST in a NA sample; (2) determine the impact of a simulated visual impairment on the IReST; (3) to examine the impact of language fluency on reading speeds on the IReST; and (4) to determine the impact of not following the IReST administration protocols on reading speeds on the IReST. It was hypothesized that: (1) the reading speeds of a NA English speaking sample would fall outside of the normative values established by the UK English IReST; (2) that a simulated visual impairment would result in further decreases in reading speed on the IReST; (3) that a multilingual sample of English speaking NAs would read the texts significantly slower than individuals whose first and dominant language was English; and finally, (4) that not following the IReST administration protocols would significantly impact the results of the assessment.

These hypotheses were examined through three different experiments. Experiment 1 found that the reading speeds of a NA English speaking sample fell below the normative values of the UK English IReST. More specifically, a sample of NA English speakers read the IReST texts on average $23 \mathrm{wpm}$ slower than the UK sample. Whereas, participants in the simulated impairment condition read the texts on average $57 \mathrm{wpm}$ slower than the UK sample. There were inconsistent results between the NHST and Bayesian analyses in Experiment 2, which examined the impact of language fluency on the IReST. Nonetheless, based on the NHST and overlapping 95\% confidence intervals of the reading speeds of the multilingual English speakers and the normative values of the NA English IReST, there were no significant differences between the reading speeds of these sample for the majority of the IReST texts in both the normal and simulated impairment conditions. Finally, Experiment 3 examined the impact of improper administration protocols on reading speeds on the IReST. The results showed that improper administration protocols significantly reduced the reading speeds on the IReST by an average of $24 \mathrm{wpm}$ in the normal vision condition. While in the simulated visual impairment condition, reading speeds were reduced by an average of $55 \mathrm{wpm}$ compared to the reading speeds of the normative values of the NA English IReST. The results of Experiment 3 also found that while 
improper administration procedures significantly reduced reading speeds on the IReST, the reduction in reading speed was not as severe as the impact of a simulated visual impairment on its own. However, when improper administration of the IReST was coupled with a simulated visual impairment, this sharply reduced the reading speeds of participants on the IReST (55 wpm).

The findings of this study suggest that the normative values of the UK English IReST are not valid in an English-speaking NA sample. Therefore, when assessing individuals reading speeds in NA, researchers and clinicians should use the values established in this study for a normative point of comparison. Furthermore, when assessing individuals with sensory impairments, researchers and clinicians should use the normative values established in the simulated impairment condition of Experiment 1 only as a guide; as there were significant differences between the reading speeds of the simulated impairment condition in Experiment 1 and actual older adults with sensory impairments. As discussed in Experiments 2, based on the results of this study it would appear that the English language IReST is robust enough to account for differences in language background and fluency when assessing individuals in their nondominant language if they are highly fluent in the language of assessment. However, it should be stressed that while this may have represented a minor deviation in standardization protocols in this study, to obtain the most accurate measure of an individual's reading speed, clients should whenever possible be assessed in their dominant language. Finally, the results of Experiment 3 demonstrate the importance of following the administration protocols of not only the IReST, but also assessments in general. It is important for researchers and clinicians to follow the standardization procedures of the IReST as it is clear that changing these procedures represents an extensive deviation from the administration protocols. More importantly, it is crucial when assessing individuals with sensory impairments that the administration protocols of the IReST are followed as the combination of a sensory impairment and improper administration of the IReST resulted in the harshest reduction in reading speed across all three experiments.

As this study only validated the IReST on sample of English speaking NA between the ages of 18 and 45, future studies should determine if the reading speeds of older adults (50+) fall outside of the normative values of the NA English IReST established in this study. I would hypothesize that the reading speeds of older adults would fall below of the normative values established in the NA English IReST. A recent study by Brussee, van Nispen, and van Rens 
(2016) found that factors such as decreased contrast sensitivity and lower levels of education in older adults impact reading speeds on the IReST. Therefore, future studies should assess the impact of sensory impairments in older adults with sensory impairments as well. Similarly, future studies should examine the impact of language fluency on the IReST by assessing solely L2 or L3 English speakers to determine if the reading speeds of these samples would fall below the normative values of the NA English IReST. Future studies should also develop NA versions of the UK IReST to address whether or not the observed differences in reading speeds in NA and UK are due to regional differences or due to the semantic content of the texts. These NA English texts should focus on identifying, and subsequently changing the grammar and syntax in the UK English IReST that is unfamiliar to NA English speakers. If changing the UK grammar and syntax does not alter the reading speeds of a NA sample, then it may provide evidence that there are actually regional differences in the ways the English language is processed.

The results of this MA thesis indicate that the normative values of the UK English IReST are not valid in an English-speaking NA sample. Additionally, as the reading speeds of a sample of NA English speakers with a simulated impairment had significantly lower reading speeds compared to normally sighted individuals, it is likely that individuals with true visual impairments will also have significantly slower reading speeds on the IReST. Therefore, researchers and clinicians who use the IReST when assessing individuals with sensory impairments will need to take this into consideration when comparing the reading speeds of visually impaired individuals to the normative values of the IReST. In clinical settings, it is also important to, whenever possible, assess individuals in their first and dominant language on the IReST. However, if this is not possible then clinicians should assess clients on the English IReST only if they report that they are very good or fluent English readers. Finally, it is crucial that clinicians and researchers precisely follow the IReST administration protocols, as not doing so will significantly reduce the measured reading speeds of clients and participants. 


\section{References}

Ahn, S. J., \& Legge, G. E. (1995). Psychophysics of reading--XIII. Predictors of magnifier-aided reading speed in low vision. Vision Research, 35(13), 1931-8. http://doi.org/10.1016/00426989(94)00293-U

Altpeter, E. K., Marx, T., Nguyen, N. X., Naumann, A., \& Trauzettel-Klosinski, S. (2015).

Measurement of reading speed with standardized texts: a comparison of single sentences and paragraphs. Graefe's Archive for Clinical and Experimental Ophthalmology, 253(8), 1369-1375. http://doi.org/10.1007/s00417-015-3065-4

Alvarado, R., Ruef, M., \& Schrank, F. (2005). Woodcock-Muñoz Language Survey-Revised. Itasca, IL: Riverside Publishing.

Attebo, K., Mitchell, P., \& Smith, W. (1996). Visual acuity and the causes of visual loss in Australia. The Blue Mountains Eye Study. Ophthalmology, 103(3), 357-64. http://doi.org/10.1016/S0161-6420(96)30684-2

Bach, M. (1996). The Freiburg Visual Acuity Test-Automatic Measurement of Visual Acuity. Optometry and Vision Science : Official Publication of the American Academy of Optometry, 73(1), 49-53. http://doi.org/10.1097/00006324-199601000-00008

Bailey, I. L., \& Lovie, J. E. (1980). The design and use of a new near-vision chart. Optometry and Vision Science, 57(6), 378-387. http://doi.org/10.1097/00006324-198006000-00011

Binns, A. M., Bunce, C., Dickinson, C., Harper, R., Tudor-Edwards, R., Woodhouse, M., ... Margrain, T. H. (2012). How effective is low vision service provision? A systematic review. Survey of Ophthalmology, 57(1), 34-65. http://doi.org/10.1016/j.survophthal.2011.06.006

Blackmore-Wright, S., Georgeson, M. a., \& Anderson, S. J. (2013). Enhanced text spacing improves reading performance in individuals with macular disease. PLoS ONE, 8(11), 1-12. http://doi.org/10.1371/journal.pone.0080325

Bourne, R. R. A., Jonas, J. B., Flaxman, S. R., Keeffe, J., Leasher, J., Naidoo, K., ... Taylor, H. R. (2014). Prevalence and causes of vision loss in high-income countries and in Eastern and Central Europe: 1990-2010. British Journal of Ophthalmology, 98(5), 629-638. http://doi.org/10.1136/bjophthalmol-2013-304033

Brussee, T., van Nispen, R. M. A., Klerkx, E. M. F. J., Knol, D. L., \& van Rens, G. H. M. B. (2015). Comparison of reading performance tests concerning difficulty of sentences and paragraphs and their reliability. Ophthalmic and Physiological Optics, 35(3), 324-335. 
http://doi.org/10.1111/opo.12204

Brussee, T., van Nispen, R. M. a., \& van Rens, G. H. M. B. (2014). Measurement properties of continuous text reading performance tests. Ophthalmic and Physiological Optics, 34(6), 636-657. http://doi.org/10.1111/opo.12158

Brussee, T., van Nispen, R. M., \& van Rens, G. H. (2016). Visual and personal characteristics are associated with reading performance in normally sighted adults. Clinical and Experimental Optometry, 1-8. http://doi.org/10.1111/cxo.12482

Carver, R. P. (1992). Reading Rate: Theory, Research, and Practical Implications. Journal of Reading, 36(2), 84-95. Retrieved from http://www.jstor.org/stable/40016440

Cheong, A. M., Lovie-Kitchin, J. E., \& Bowers, A. R. (2002). Determining magnification for reading with low vision. Clinical and Experimental Optometry, 85(4), 229-237. http://doi.org/10.1111/j.1444-0938.2002.tb03042.x

Cop, U., Drieghe, D., \& Duyck, W. (2015). Eye Movement Patterns in Natural Reading: A Comparison of Monolingual and Bilingual Reading of a Novel. PloS One, 10(8), e0134008. http://doi.org/10.1371/journal.pone.0134008

Corn, A., \& Koenig, A. (1996). Foundations of Low Vision: Clinical and functional perspectives (2nd Editio). New York: American Foundation for the Blind Press.

Crossland, M. D., Culham, L. E., \& Rubin, G. S. (2004). Fixation stability and reading speed in patients with newly developed macular disease*. Ophthalmic and Physiological Optics, 24(4), 327-333. http://doi.org/10.1111/j.1475-1313.2004.00213.x

Den Brinker, B. P. L. M., \& Bruggeman, H. (1996). Visual requirements for reading: The importance of a large field of view in reading with a magnifier. Journal of Videology, 1(1), 27-38. Retrieved from

http://citeseerx.ist.psu.edu/viewdoc/download?doi=10.1.1.93.9704\&amp;rep=rep1\&amp;ty $\mathrm{pe}=\mathrm{pdf}$

Dienes, Z. (2011). Bayesian Versus Orthodox Statistics: Which Side Are You On? Perspectives on Psychological Science, 6(3), 274-290. http://doi.org/10.1177/1745691611406920

Elliott, D. B., Bullimore, M. A., Patla, A. E., \& Whitaker, D. (1996). Effect of a cataract simulation on clinical and real world vision. British Journal of Ophthalmology, 80(9), 799804. http://doi.org/10.1136/bjo.80.9.799

Elliott, D. B., Trukolo-Ilic, M., Strong, J. G., Pace, R., Plotkin, A., \& Bevers, P. (1997). 
Demographic characteristics of the vision-disabled elderly. Investigative Ophthalmology \& Visual Science, 38(12), 2566-75. Retrieved from

http://www.ncbi.nlm.nih.gov/pubmed/9375576

Favreau, M., \& Segalowitz, N. S. (1983). Automatic and controlled processes in the first- and second-language reading of fluent bilinguals. Memory \& Cognition, 11(6), 565-574. http://doi.org/10.3758/BF03198281

Ferris, F. L., Kassoff, A., Bresnick, G. H., \& Bailey, I. (1982). New Visual Acuity Charts for Clinical Research. American Journal of Ophthalmology, 94(1), 91-96. http://doi.org/10.1016/0002-9394(82)90197-0

Fine, E. M., \& Peli, E. (1996). The Role of Context in Reading with Central Field Loss. Optometry and Vision Science, 73(8), 533-539. http://doi.org/10.1097/00006324199608000-00003

Fine, E. M., Rubin, G. S., Hazel, C. A., \& Petre, K. L. (1999). Are the Benefits of Sentence Context Different in Central and Peripheral Vision? Optometry and Vision Science, 76(11), 764-769. http://doi.org/10.1097/00006324-199911000-00025

Fosse, P. (2005). Psychophysics of reading and illumination needs during reading in visually impaired subjects with age-related macular degeneration. Norwegian University of Science and Technology. Retrieved from http://www.divaportal.org/smash/record.jsf?pid=diva2\%3A126091\&dswid=-1000

Friedman, S. M., Munoz, B., Rubin, G. S., West, S. K., Bandeen-Roche, K., \& Fried, L. P. (1999). Characteristics of discrepancies between self-reported visual function and measured reading speed. Salisbury Eye Evaluation Project Team. Investigative Ophthalmology \& Visual Science, 40(5), 858-64. Retrieved from http://www.researchgate.net/publication/13189540_Characteristics_of_discrepancies_betwe en_selfreported_visual_function_and_measured_reading_speed._Salisbury_Eye_Evaluation_Proje ct_Team\npapers2://publication/uuid/130ACF36-58C8-4118-A458-90E2A85B6182

Gao, X., \& Loomes, M. (2016). A new approach to image enhancement for the visually impaired. Electronic Imaging, 2016(20), 1-7. http://doi.org/10.2352/ISSN.24701173.2016.20.COLOR-325

Gasquoine, P. G., Croyle, K. L., Cavazos-Gonzalez, C., \& Sandoval, O. (2007). Language of 
administration and neuropsychological test performance in neurologically intact Hispanic American bilingual adults. Archives of Clinical Neuropsychology : The Official Journal of the National Academy of Neuropsychologists, 22(8), 991-1001. http://doi.org/10.1016/j.acn.2007.08.003

Gollan, T. H., Montoya, R. I., Cera, C., \& Sandoval, T. C. (2008). More use almost always means a smaller frequency effect: Aging, bilingualism, and the weaker links hypothesis t $^{2}$. Journal of Memory and Language, 58(3), 787-814.

http://doi.org/10.1016/j.jml.2007.07.001

Google Ngram Viewer. (n.d.). Retrieved February 7, 2017, from https://books.google.com/ngrams/graph?content=greengrocer\%2C+shopkeeper\&year_start $=1800 \&$ year_end $=2010 \&$ corpus $=15 \&$ smoothing $=3 \&$ share $=\&$ direct_url $=t 1 \% 3 \mathrm{~B} \% 2 \mathrm{Cgreeng}$ rocer $\% 3 \mathrm{~B} \% 2 \mathrm{Cc} 0 \% 3 \mathrm{~B} . \mathrm{t} 1 \% 3 \mathrm{~B} \% 2 \mathrm{Cshopkeeper} \% 3 \mathrm{~B} \% 2 \mathrm{Cc} 0$

Guralnik, J. M., Branch, L. G., Cummings, S. R., \& Curb, J. D. (1989). Physical performance measures in aging research. Journal of Gerontology, 44(5), M141-M146.

Hahn, G. a, Penka, D., Gehrlich, C., Messias, a, Weismann, M., Hyvärinen, L., ... TrauzettelKlosinski, S. (2006). New standardised texts for assessing reading performance in four European languages. The British Journal of Ophthalmology, 90(4), 480-484. http://doi.org/10.1136/bjo.2005.087379

Hart, P. M., Chakravarthy, U., Stevenson, M. R., \& Jamison, J. Q. (1999). A vision specific functional index for use in patients with age related macular degeneration. British Journal of Ophthalmology, 83(10), 1115-1120. http://doi.org/10.1136/bjo.83.10.1115

Holladay, J. T., \& Msee. (2004). Visual acuity measurements. Journal of Cataract \& Refractive Surgery, 30(2), 287-290. http://doi.org/10.1016/j.jcrs.2004.01.014

Hoover, W. A., \& Gough, P. B. (1990). The simple view of reading. Reading and Writing, 2(2), 127-160. http://doi.org/10.1007/BF00401799

Jacewicz, E., Fox, R. A., O’Neill, C., \& Salmons, J. (2009). Articulation rate across dialect, age, and gender. Language Variation and Change, 21(2), 233. http://doi.org/10.1017/S0954394509990093

JASP Team. (2016). JASP (Version 0.8.0.1). Retrieved from https://jasp-stats.org/ Kline, R. B. (2013). Beyond significance testing : statistics reform in the behavioral sciences (Second Edi). American Psychological Association. 
Kruschke, J. K. . (2014). Doing Bayesian data analysis : a tutorial with R, JAGS, and Stan (2nd ed.). Academic Press.

Kurtenbach, A., Langrová, H., Messias, A., Zrenner, E., \& Jägle, H. (2013). A comparison of the performance of three visual evoked potential-based methods to estimate visual acuity. Documenta Ophthalmologica, 126(1), 45-56. http://doi.org/10.1007/s10633-012-9359-5

Lebowitz, B. K., Weinstein, C., Beiser, A., Seshadri, S., Wolf, P. A., Auerbach, S., \& Au, R. (2015). Lifelong Reading Disorder and Mild Cognitive Impairment: Implications for Diagnosis. Journal of Alzheimer's Disease, 50(1), 41-45. http://doi.org/10.3233/JAD150543

Lee, J., \& Schallert, D. L. (1997). The Relative Contribution of L2 Language Proficiency and L1 Reading Ability to L2 Reading Performance: A Test of the Threshold Hypothesis in an EFL Context. TESOL Quarterly, 31(4), 713. http://doi.org/10.2307/3587757

Legge, G. E., Ross, J. A., Luebker, A., \& LaMay, J. M. (1989). Psychophysics of reading. VIII. The Minnesota Low-Vision Reading Test. Optometry and Vision Science : Official Publication of the American Academy of Optometry, 66(12), 843-53. Retrieved from http://www.ncbi.nlm.nih.gov/pubmed/2626251

Legge, G. E., Rubin, G. S., Pelli, D. G., \& Schleske, M. M. (1985). Psychophysics of reading--II. Low vision. Vision Research, 25(2), 253-65. http://doi.org/10.1016/0042-6989(85)90118-X

Lezak, M. D., Howieson, D. B., Bigler, E. D., \& Tranel, D. (2012). Neuropsychological assessment (5th ed.). New York: Oxford University Press.

Loftus, G. R., \& Masson, M. E. J. (1994). Using confidence intervals in within-subject designs. Psychonomic Bulletin \& Review, 1(4), 476-490. http://doi.org/10.3758/BF03210951

Mangione, C. M., Phillips, R. S., Seddon, J. M., Lawrence, M. G., Cook, E. F., Dailey, R., \& Goldman, L. (1992). Development of the "Activities of Daily Vision Scale”. A measure of visual functional status. Medical Care, 30(12), 1111-26. Retrieved from http://www.ncbi.nlm.nih.gov/pubmed/1453816

Mansfield, J. S., Ahn, S. J., Legge, G., \& Luebker, A. (1993). A new reading-acuity chart for normal and low vision. Noninvasive assessment of the visual system: summaries. Technical digest series. Optical Society of America.

Massof, R. (1995). A systems model for low vision rehabilitation. I. Basic concepts., 72(10), 725-736. 
McAllister, B., \& Kammer, R. (2014). Low Vision Rehabilitation. In Ophthalmology - Current Clinical and Research Updates (pp. 347-381). InTech. http://doi.org/10.5772/58436

Morrice, E., Johnson, A. P., Marinier, J.-A., \& Wittich, W. (2017). Assessment of the Apple iPad as a low-vision reading aid. Eye. JOUR. http://doi.org/10.1038/eye.2016.309

Nguyen, N. X., Stockum, A., Hahn, G. A., \& Trauzettel-Klosinski, S. (2011). Training to improve reading speed in patients with juvenile macular dystrophy: a randomized study comparing two training methods. Acta Ophthalmologica, 89(1), e82-e88. http://doi.org/10.1111/j.1755-3768.2010.02081.x

Nguyen, N. X., Weismann, M., \& Trauzettel-Klosinski, S. (2009). Improvement of reading speed after providing of low vision aids in patients with age-related macular degeneration. Acta Ophthalmologica, 87(8), 849-853. http://doi.org/10.1111/j.1755-3768.2008.01423.x

Overbury, O., \& Wittich, W. (2011). Barriers to Low Vision Rehabilitation: The Montreal Barriers Study. Investigative Opthalmology \& Visual Science, 52(12), 8933. http://doi.org/10.1167/iovs.11-8116

Palmer, J., MacLeod, C. M., Hunt, E., \& Davidson, J. E. (1985). Information processing correlates of reading. Journal of Memory and Language, 24(1), 59-88. http://doi.org/10.1016/0749-596X(85)90016-6

Pascolini, D., \& Mariotti, S. P. (2011). Global estimates of visual impairment: 2010. British Journal of Ophthalmology. http://doi.org/10.1136/bjophthalmol-2011-300539

Pell, G., Homer, M. S., \& Roberts, T. E. (2008). Assessor training: its effects on criterion-based assessment in a medical context. International Journal of Research \& Method in Education, 31(2), 143-154. http://doi.org/10.1080/17437270802124525

Radner, W., Willinger, U., Obermayer, W., Mudrich, C., Velikay-Parel, M., \& Eisenwort, B. (1998). Eine neue Lesetafel* zur gleichzeitigen Bestimmung von Lesevisus und Lesegeschwindigkeit. Klinische Monatsblätter Für Augenheilkunde, 213(9), 174-181. http://doi.org/10.1055/s-2008-1034969

Regie de l'assurance maladie du Quebec/RAMQ. (2006). Services covered in Quebec - Visual devices.

Robillard, N., \& Overbury, O. (2006). Quebec model for low vision rehabilitation. Canadian Journal of Ophthalmology. Journal Canadien D'ophtalmologie, 41(3), 362-366. http://doi.org/10.1139/I06-022 
Rousek, J. B., \& Hallbeck, M. S. (2011). Improving and analyzing signage within a healthcare setting. Applied Ergonomics, 42(6), 771-784. http://doi.org/10.1016/j.apergo.2010.12.004

Rubin, G. S. (2013). Measuring reading performance. Vision Research, 90, 43-51. http://doi.org/10.1016/j.visres.2013.02.015

Runge, P. E. (2000). Eduard Jaeger's Test-Types (Schrift-Scalen) and the historical development of vision tests. Transactions of the American Ophthalmological Society, 98, 375-438. Retrieved from http://www.ncbi.nlm.nih.gov/pubmed/11190034

Sass, S. M., Legge, G. E., \& Lee, H.-W. (2006). Low-vision reading speed: influences of linguistic inference and aging. Optometry and Vision Science : Official Publication of the American Academy of Optometry, 83(3), 166-177. http://doi.org/10.1097/01.opx.0000204752.43520.17

Sattler, J. M. (1969). Effects of cues and examiner influence on two Wechsler subtests. Journal of Consulting and Clinical Psychology, 33(6), 716-721. http://doi.org/10.1037/h0028484

Sattler, J. M. (2008). Assessment of Children : Cognitive Foundations. San Diego: J.M. Sattler. Retrieved from http://clues.concordia.ca/search/i0970267142

Schulze-Bonsel, K., Feltgen, N., Burau, H., Hansen, L., \& Bach, M. (2006). Visual Acuities "Hand Motion" and "Counting Fingers" Can Be Quantified with the Freiburg Visual Acuity Test. Investigative Opthalmology \& Visual Science, 47(3), 1236. http://doi.org/10.1167/iovs.05-0981

Segalowitz, N., \& Hulstijn, J. (2005). Automaticity in bilingualism and second language learning. In J. F. Kroll \& A. M. B. De Groot (Eds.), Handbook of bilingualism: Psycholinguistic approaches (pp. 371-388). Oxford: Oxford University Press.

Seiple, W., Grant, P., \& Szlyk, J. P. (2011). Reading Rehabilitation of Individuals with AMD: Relative Effectiveness of Training Approaches. Investigative Opthalmology \& Visual Science, 52(6), 2938. http://doi.org/10.1167/iovs.10-6137

Seliger, H. W. (1972). Improving Reading Speed and Comprehension in English as a Second Language. ELT Journal, XXVII(1), 48-55. http://doi.org/10.1093/elt/XXVII.1.48

Sloane, M. E., Owsley, C., \& Bruni, J. R. (1992). Visual Activities Questionnaire (VAQ) The Visual Activities Questionnaire: Developing an instrument for assessing problems in everyday visual tasks. Topical Meeting of the Optical Society of America.

Southall, K., \& Wittich, W. (2012). Barriers to Low Vision Rehabilitation: A Qualitative 
Approach. Journal of Visual Impairment \& Blindness, 106(5), 261-274. Retrieved from http://search.ebscohost.com/login.aspx?direct=true\&AuthType=ip,url,uid,shib\&db=rzh\&A $\mathrm{N}=2011557854 \&$ site $=$ ehost-live

Statistics Canada. (2012). Focus on Geography Series, 2011 Census - Census metropolitan area of Montréal. Retrieved February 21, 2017, from https://www12.statcan.gc.ca/censusrecensement/2011/as-sa/fogs-spg/Facts-cma-eng.cfm?LANG=Eng\&GK=CMA\&GC=462

Statistics Canada. (2017). The evolution of English - French bilingualism in Canada from 1901 to 2011. Retrieved March 25, 2017, from http://www.statcan.gc.ca/pub/11-630-x/11-630x2016001-eng.htm

Stelmack, J. (2001). Quality of Life of Low-Vision Patients and Outcomes of Low-Vision Rehabilitation. Optometry and Vision Science, 78(5), 335-342. http://doi.org/10.1097/00006324-200105000-00017

Stevens, G. A., White, R. A., Flaxman, S. R., Price, H., Jonas, J. B., Keeffe, J., .. Bourne, R. R. A. (2013). Global Prevalence of Vision Impairment and Blindness. Ophthalmology, 120(12), 2377-2384. http://doi.org/10.1016/j.ophtha.2013.05.025

Trauzettel-Klosinski, S., \& Dietz, K. (2012). Standardized assessment of reading performance: The new international reading speed texts IReST. Investigative Ophthalmology and Visual Science, 53(9), 5452-5461. http://doi.org/10.1167/iovs.11-8284

Virgili, G., \& Acosta, R. (2006). Reading aids for adults with low vision. Cochrane Database of Systematic Reviews, (4). http://doi.org/10.1002/14651858.CD003303.pub2

Vision Rehabilitation Services LLC. (n.d.). Fork in the Road. Retrieved February 24, 2017, from https://www.lowvisionsimulators.com/

Watson, G. R. (2001). Low vision in the geriatric population: rehabilitation and management. Journal of the American Geriatrics Society, 49(3), 317-330. http://doi.org/10.1046/j.15325415.2001.4930317.x

Westheimer, G., Snell AC, S. S., WW, W., FW, W., Teller DY, M. R. B. R. et al, L, L., ... Bailey IL, L. J. (1979). Scaling of Visual Acuity Measurements. Archives of Ophthalmology, 97(2), 327-330. http://doi.org/10.1001/archopht.1979.01020010173020 Wetzels, R., Matzke, D., Lee, M. D., Rouder, J. N., Iverson, G. J., \& Wagenmakers, E.-J. (2011). Statistical Evidence in Experimental Psychology. Perspectives on Psychological Science, 6(3), 291-298. http://doi.org/10.1177/1745691611406923 
Whiteside, S. P. (1996). Temporal-based acoustic-phonetic patterns in read speech: some evidence for speaker sex differences. Journal of the International Phonetic Association, 26(1), 23. http://doi.org/10.1017/S0025100300005302

Whittaker, S. G., \& Lovie-Kitchin, J. (1993). Visual requirements for reading. Optometry and Vision Science : Official Publication of the American Academy of Optometry, 70(1), 54-65. Retrieved from http://www.ncbi.nlm.nih.gov/pubmed/8430009 


\section{Appendix A}

Code:

Date:

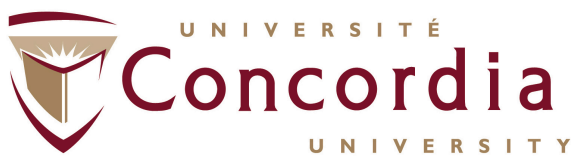

\section{LANGUAGE BACKGROUND QUESTIONNAIRE}

Name:

Age:

1. If you are a student:

What is your field of study?

What degree are you pursuing? College (Cégep/Diploma/etc.) Bachelor $\mathrm{MA} / \mathrm{PhD} / \mathrm{etc}$

2. Where were you born? City:

Country:

3. What do you consider to be your first learned language?

English

French

Other (specify)

4. What do you consider to be your second learned language?

$$
\text { English }
$$

French

Other (specify)

5. At what age did you learn your second language? Age of years old

6. What language do you consider your dominant language (the language you are most comfortable in)?

$$
\text { English _ _ French _ _ Other (specify) }
$$

7. What language do you speak at home now?

8. What is the first language of your: Mother?

Father?

9. What was the language of instruction of the school you attended? (Check all appropriate):

- Preschool:

English

French

French Immersion

Other (specify)

- Elementary school: English French

French Immersion Other (specify)

- Middle/High school:English French

- College/Cégep/Diploma: English

French Immersion Other (specify)

- University:
French

French
Other (specify)

Other (specify)

10. If you are not currently a student, what is the highest level of education you have completed:

$$
\begin{aligned}
& \text { High school_- } \\
& \text { University: Bachelor }
\end{aligned}
$$

\section{College/Cégep/Diploma}

University: $\mathrm{MA} / \mathrm{PhD} / \mathrm{etc}$. 
Code:

Date:

11. Did you receive second/foreign language instruction at any of the levels listed below YES NO

If YES, specify each language and for how long, starting with your main second language.

MAIN SECOND/FOREIGN LANGUAGE:

- Elementary School:

- Middle/High School:

- College/Cégep/Diploma/University:

- Other:

Please specify:

THIRD LANGUAGE (if any):

Any other special learning experiences (e.g., intensive French in Grade 6; long visit to France):

12. Do you have any visual impairment NOT corrected by wearing glasses or contact lenses?

13. Do you have a known hearing impairment?

14. Do you have a known reading or attention disability?

Yes

Yes

Yes

No

less than 1 yea

1-2 years more than 2 years

less than 1 year

1-2 years

ess than 1 year -2 years more than 2 years

15. What percentage of your interactions are in (total $=100 \%)$ :

$$
\text { English _ } \% \text { ? } \quad \text { French _ } \% \text { ? Other __ } \% \text { ? }
$$

16. Please rate your level of ability for each of the four skills listed below by using the following rating scheme and circling the appropriate number in the boxes below:

$$
\mathbf{1}=\text { no ability at all } \mathbf{2} \text { = elementary } \quad \mathbf{3} \text { = moderate } \quad \mathbf{4}=\text { very good } \quad \mathbf{5}=\text { fluent ability }
$$

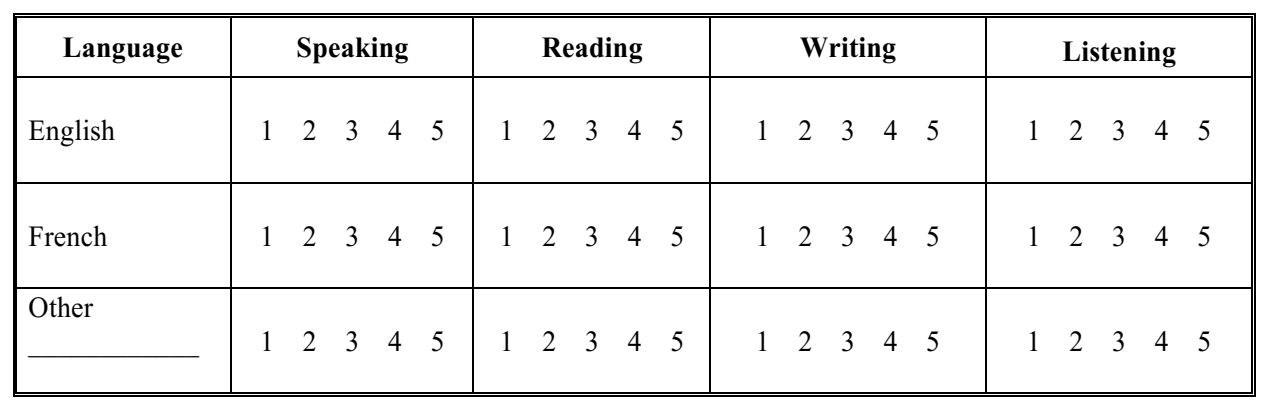


Appendix B

Proportion of Reading Comprehension Questions Answered Correctly in Experiment 1

\begin{tabular}{ccccccc}
\hline IReST & \multicolumn{3}{c}{ Impaired } & \multicolumn{3}{c}{ Normal } \\
\cline { 2 - 7 } & Correct & Incorrect & $\%$ Correct & Correct & Incorrect & $\%$ Correct \\
\hline 1 & 21 & 4 & $84 \%$ & 23 & 2 & $92 \%$ \\
2 & 18 & 7 & $72 \%$ & 13 & 12 & $52 \%$ \\
3 & 25 & 0 & $100 \%$ & 22 & 3 & $88 \%$ \\
4 & 23 & 2 & $92 \%$ & 20 & 5 & $80 \%$ \\
5 & 24 & 1 & $96 \%$ & 24 & 1 & $96 \%$ \\
6 & 24 & 1 & $96 \%$ & 25 & 0 & $100 \%$ \\
7 & 20 & 5 & $80 \%$ & 19 & 6 & $76 \%$ \\
8 & 24 & 1 & $96 \%$ & 25 & 0 & $100 \%$ \\
9 & 18 & 7 & $72 \%$ & 20 & 5 & $80 \%$ \\
10 & 25 & 0 & $100 \%$ & 24 & 1 & $96 \%$ \\
\hline
\end{tabular}


Appendix C

Proportion of Reading Comprehension Questions Answered Correctly in Experiment 2

\begin{tabular}{ccccccc}
\hline \multirow{2}{*}{ IReST } & \multicolumn{3}{c}{ Impaired } & \multicolumn{3}{c}{ Normal } \\
\cline { 2 - 7 } & Correct & Incorrect & $\%$ Correct & Correct & Incorrect & $\%$ Correct \\
\hline 1 & 22 & 3 & $88 \%$ & 22 & 3 & $88 \%$ \\
2 & 15 & 10 & $60 \%$ & 15 & 10 & $60 \%$ \\
3 & 19 & 6 & $76 \%$ & 19 & 6 & $76 \%$ \\
4 & 21 & 4 & $84 \%$ & 19 & 6 & $76 \%$ \\
5 & 23 & 2 & $92 \%$ & 24 & 1 & $96 \%$ \\
6 & 23 & 2 & $92 \%$ & 21 & 4 & $84 \%$ \\
7 & 18 & 7 & $72 \%$ & 20 & 5 & $80 \%$ \\
8 & 25 & 0 & $100 \%$ & 22 & 3 & $88 \%$ \\
9 & 16 & 9 & $64 \%$ & 11 & 14 & $44 \%$ \\
10 & 25 & 0 & $100 \%$ & 23 & 2 & $92 \%$ \\
\hline
\end{tabular}




\section{Appendix D}

Proportion of Reading Comprehension Questions Answered Correctly in Experiment 3

\begin{tabular}{ccccccc}
\hline \multirow{2}{*}{ IReST } & \multicolumn{3}{c}{ Impaired } & \multicolumn{3}{c}{ Normal } \\
\cline { 2 - 7 } & Correct & Incorrect & $\%$ Correct & Correct & Incorrect & $\%$ Correct \\
\hline 1 & 21 & 4 & $84 \%$ & 19 & 6 & $76 \%$ \\
2 & 19 & 6 & $76 \%$ & 15 & 10 & $60 \%$ \\
3 & 22 & 3 & $88 \%$ & 18 & 7 & $72 \%$ \\
4 & 20 & 5 & $80 \%$ & 22 & 3 & $88 \%$ \\
5 & 24 & 1 & $96 \%$ & 24 & 1 & $96 \%$ \\
6 & 22 & 3 & $88 \%$ & 24 & 1 & $96 \%$ \\
7 & 20 & 5 & $80 \%$ & 22 & 3 & $88 \%$ \\
9 & 25 & 0 & $100 \%$ & 25 & 0 & $100 \%$ \\
10 & 19 & 6 & $76 \%$ & 11 & 14 & $44 \%$ \\
\hline
\end{tabular}

